\title{
Manual on financial mechanism for the health facilities: Introducing pay-for-performance approach to increase utilization of maternal, newborn, and child health services in Bangladesh
}

\author{
Laila Rahman \\ Population Council \\ Dipak Kumar Shil \\ Population Council \\ Md. Mamun-or Rashid \\ Population Council \\ Ismat Ara Hena \\ Population Council \\ Md. Noorunnabi Talukder \\ Population Council \\ Follow this and additional works at: https://knowledgecommons.popcouncil.org/departments_sbsr-rh

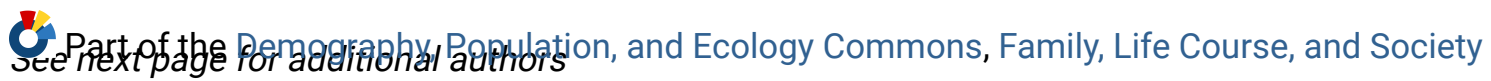 \\ Commons, Gender and Sexuality Commons, International Public Health Commons, Maternal and Child \\ Health Commons, Medicine and Health Commons, and the Women's Health Commons \\ How does access to this work benefit you? Let us know!
}

\section{Recommended Citation}

Rahman, Laila, Dipak Kumar Shil, Md. Mamun-or Rashid, Ismat Ara Hena, Md. Noorunnabi Talukder, Farhana Akter, Anup Kumar Dey, Ripa Ali, Joynal Abedin, Mursheda Rahman, Md. Ataur Rahman, Md. Julkarnayeen, Arifur Rahman, and Md. Abdur Rab Sardar. 2010. "Manual on financial mechanism for the health facilities: Introducing pay-for-performance approach to increase utilization of maternal, newborn, and child health services in Bangladesh." Dhaka: Population Council. 


\section{Authors}

Laila Rahman, Dipak Kumar Shil, Md. Mamun-or Rashid, Ismat Ara Hena, Md. Noorunnabi Talukder, Farhana Akter, Anup Kumar Dey, Ripa Ali, Joynal Abedin, Mursheda Rahman, Md. Ataur Rahman, Md. Julkarnayeen, Arifur Rahman, and Md. Abdur Rab Sardar 


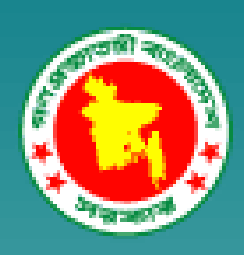

Manual on Financial Mechanism for the Health Facilities

\author{
Introducing Pay-for-Performance (P4P) Approach to \\ Increase Utilization of Maternal, Newborn and \\ Child Health Services in Bangladesh
}

Population Council, Bangladesh

November 2010

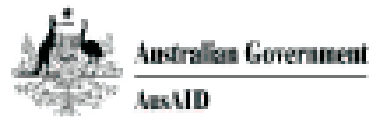

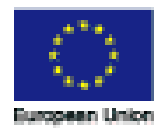
(1) Population Counal unicef(3) 


\title{
Manual on Financial Mechanism for the Health Facilities \\ For Pilot Study on Introducing Pay-For-Performance (P4P) Approach to Increase Utilization of Maternal, Newborn and Child Health Services in Bangladesh
}

\author{
Laila Rahman \\ Dipak Kumar Shil \\ Mamun-or-Rashid \\ Ismat Ara Hena \\ Md. Noorunnabi Talukder \\ Farhana Akter \\ Anup Kumar Dey \\ Ripa Ali \\ Joynal Abedin \\ Mursheda Rahman \\ Md. Ataur Rahman \\ Md. Julkarnayeen \\ Arifur Rahman \\ Md. Abdur Rab Sardar
}

Population Council, Bangladesh

November 2010

Special acknowledgement:

Md. Delwar Hossen, Chartered Accountant, Hossen Delwar \& Co. Chartered Accountants, Ubaidur Rob, Country Director, Population Council, A.K.M. Zafar Ullah Khan, Adviser, Population Council, for reviewing the manual.

This publication is made possible through the financial support of UNICEF/Bangladesh. The opinions expressed herein are those of the authors and do not reflect the views of the Population Council and UNICEF/Bangladesh. 
Suggested citation:

Rahman, Laila, Dipak Kumar Shil, Mamun-or-Rashid, Ismat Ara Hena, Md. Noorunnabi Talukder, Farhana Akter, Anup Kumar Dey, Ripa Ali, Joynal Abedin, Mursheda Rahman, Md. Ataur Rahman, Md. Julkarnayeen, Arifur Rahman, and Md. Abdur Rab Sardar. 2010. "Manual on financial mechanism for the bealth facilities: Introducing pay-for-performance approach to increase utilization of maternal, newborn, and child bealth services in Bangladesh." Dhaka, Bangladesh: Population Council. 


\section{Abbreviations}

$\begin{array}{ll}\text { BEmONC } & \text { Basic Emergency Obstetric and Newborn Care } \\ \text { CEmONC } & \text { Comprehensive Emergency Obstetric and Newborn Care } \\ \text { CS } & \text { Civil Surgeon } \\ \text { DCM } & \text { Drugs, Consumables and Maintenance } \\ \text { DGHS } & \text { Directorate General of Health Services } \\ \text { DTC } & \text { Diagnostic Testing Center } \\ \text { EmONC } & \text { Emergency Obstetric and Newborn Care } \\ \text { FWA } & \text { Family Welfare Assistant } \\ \text { GoB } & \text { Government of Bangladesh } \\ \text { HA } & \text { Health Assistant } \\ \text { MNCH } & \text { Maternal, Newborn and Child Health } \\ \text { MNH } & \text { Maternal and Newborn Health } \\ \text { NGO } & \text { Non-governmental Organization } \\ \text { P4P } & \text { Pay-for-Performance } \\ \text { QAG } & \text { Quality Assurance Group } \\ \text { QAT } & \text { Quality Assurance Team } \\ \text { RMO } & \text { Resident Medical Officer } \\ \text { UHC } & \text { Upazila Health Complex } \\ \text { UFPO } & \text { Upazila Family Planning Officer } \\ \text { UHFPO } & \text { Upazila Health and Family Planning Officer } \\ \text { UNICEF } & \text { United Nations Children's Fund } \\ \text { WHO } & \text { World Health Organization } \\ & \end{array}$




\section{Table of Contents}

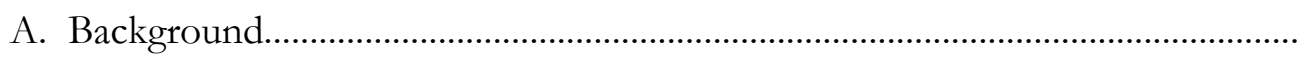

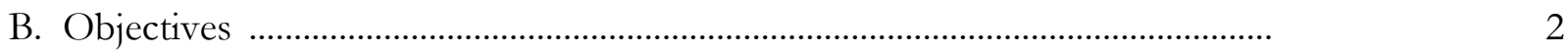

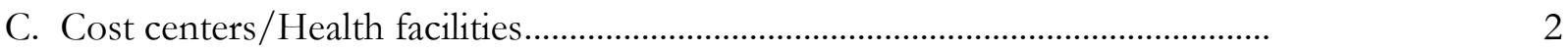

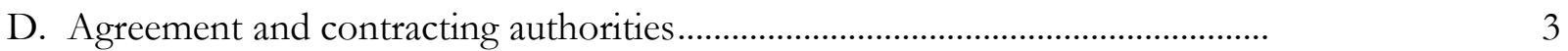

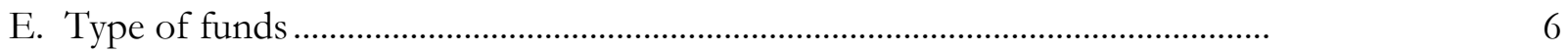

F. Requisite documents for receiving fund in advance, incurring expenses, and 6

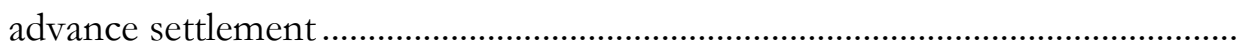

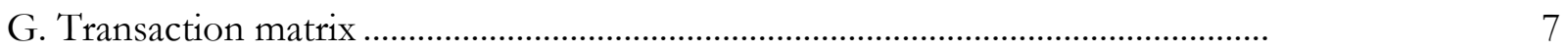

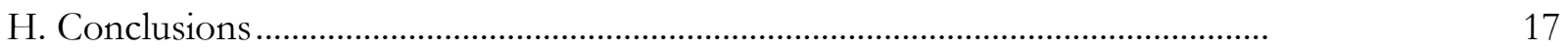

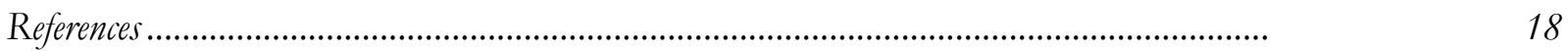

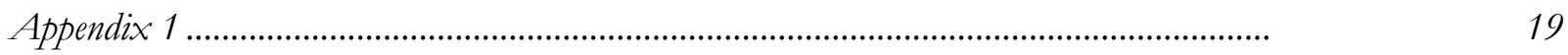




\section{A. Background}

The Population Council (the Council) initiated an operations research study (pilot study) to test two Pay-for-Performance (P4P) strategies to improve maternal, newborn and child health (MNCH) services in Bangladesh in mid-February 2010 (Population Council 2010). The project's intervention activities have started from October 2010; and the study is scheduled to end in June 2011 unless the project is extended to complete at least a 12-month intervention period. The Manual on Financial Mechanism of the Health Facilities depicts the guidelines, procedures, tools, institutions, and actors to receive fund and incur expenses for the health facilities in carrying out intervention activities to improve MNCH services.

The P4P study is being implemented as a part of the two on-going $\mathrm{MNCH}$ and maternal and newborn health $(\mathrm{MNH})$ projects of the United Nations Children's Fund (UNICEF) implemented by the Directorate General of Health Services (DGHS), Government of Bangladesh (GoB). The study has been envisaged as a human resource innovation project under the Operational Plan of the GoB for the year July 2010 to June 2011. Existing non-governmental organization (NGO) partners of the $\mathrm{MNCH}$ and MNH projects are partners of the project while the James P. Grant School of Public Health of BRAC University has been collaborating with the Council. The study has been testing two strategies. The first strategy introduces incentives tied with performance for motivating service providers to improve the quantity as well as quality of services; and enable the poor pregnant women, and mothers of newborns and under-five children to access services by reducing their outof-pocket costs for medicines, transportation and incidental costs through subsidized coupons. The second strategy constitutes a $\mathrm{P} 4 \mathrm{P}$ scheme for providers only. Both the strategies assume: facility strengthening by UNICEF in terms of human resource development and replenishing with necessary equipment and supplies, and community awareness by NGO partners constant. Gaibandha and Kurigram districts are implementing the first strategy, Jamalpur is implementing the second strategy, and Thakurgaon is the control site (Population Council 2010).

In order to implement the P4P approach and coupon distribution, the study outlined the formation of facility-based P4P and/or Coupon Committee ${ }^{1}$ (The Committee), Quality Assurance Teams (QATs) and Quality Assurance Groups (QAGs). The P4P and/or Coupon Committee of the selected facilities will implement the intervention activities with assistance from the facility-based QATs while the QAGs, formed in membership of experts consisting of professionals from nearby Medical College Hospitals and the Obstetrics and Gynecological Society of Bangladesh, make quarterly visits to accredit the facility, measure the performance of the facility-based MNCH teams and mentor the QATs on performance improvement. The P4P and/or Coupon Committees, QATs and QAGs are important actors to implement the P4P approach and coupon utilization for the poor pregnant women, newborn and under-five children. The P4P Committees and/or Coupon Committees will engage and guide the QATs who will identify the gaps and needs of the facility, develop team-based action plans to improve the services, make the facilities functional, provide quality $\mathrm{MNCH}$ services, and ensure achievement of qualitative and quantitative targets. In addition to fund for incentives and coupons, a small amount of fund, namely, Drugs, Consumables and Maintenance (DCM) and Other Fund will be provided to the facilities. The P4P and/or Coupon Committees will utilize this fund to keep the facilities functional in case of any supply constraint in

\footnotetext{
${ }^{1}$ The P4P and/or Coupon Committee is known as 'P4P and Coupon Committee' in Gaibandha and Kurigram; and 'P4P Committee' in Jamalpur.
} 
essential service drugs and/or address any immediate consumables and maintenance need in discussion with the QATs.

Four guidelines on implementation of performance-based incentives to providers, formation of the P4P Committees and/or Coupon Committee and QATs, formation of the QAGs, and distribution and realization of coupons have been developed on the basis of five policy level and consensus building workshops under the leadership of the DGHS (DGHS, Population Council, and UNICEF 2010; Population Council 2010; Rahman et al. 2010). The guidelines have been approved by the Director, Primary Health Care \& Line Director, Essential Service Delivery of the DGHS, Ministry of Health and Family Welfare, Government of Bangladesh in October 2010.

In order to enable the facilities to implement the P4P intervention activities approved in the guidelines, the Committees need to opportunely receive and effectively utilize the fund following the standard accounting procedures. The Council has developed this Manual on Financial Mechanism for the Health Facilities and the Committees to receive fund as advance, incur expenses, and make payments to the beneficiaries, clients, and vendors following the approved guidelines, standard accounting procedures and tools mentioned in the Manual.

\section{B. Objectives}

The objective of the Manual is to describe the purposes and processes, and list of appropriate documents to enable the facilities and the P4P and/or Coupon Committees to opportunely receive and appropriately utilize the funds under the P4P and coupon mechanism to improve the $\mathrm{MNCH}$ services. The specific objectives are to enable the facilities and the Committees to:

- $\quad$ Complete fund request documentation for receiving funds on time;

- Incur expenses for incentives, coupons, drugs, consumables, maintenance and other payments;

- $\quad$ Settle advance against approved expenses following the guidelines, and standard accounting practices and procedures after auditor's certification;

- $\quad$ Prevent fraud and misappropriation of funds; and

- Take necessary remedial actions immediately in case of misappropriation of funds.

\section{Cost centers/Health facilities}

The 12 selected health facilities of the three intervention districts, Gaibandha, Kurigram and Jamalpur, are the cost centers for the P4P pilot study that will receive fund, and incur expenses until the end of the project. There are mainly two types of facilities: one provides Comprehensive Emergency Obstetric and Newborn Care (CEmONC) services, and basic EmONC services. District Hospitals and one Upazila Health Complex (UHC) from the three districts are CEmONC facilities and the remaining UHCs are basic EmONC facilities. The facilities that provide Basic Emergency Obstetric and Newborn Care are known as BEmONC facility; and facilities that provide Comprehensive Emergency Obstetric and Newborn Care are known as CEmONC facility. The difference between these two types of facilities is that the BEmONC facility provides only 7 out of 9 World Health Organization (WHO) suggested signal functions while the CEmONC facility provides 
all the 9 functions $^{2}$ (WHO 2009). The QAGs during the accreditation visit ascertain the functional status of the facilities. The selected facilities are depicted in the following table.

Table 1. Implementing facilities and the cost centers according to district

\begin{tabular}{|c|c|c|c|c|c|}
\hline \multicolumn{2}{|l|}{ Gaibandha } & \multicolumn{2}{|l|}{ Kurigram } & \multicolumn{2}{|l|}{ Jamalpur } \\
\hline Facility & $\begin{array}{l}\text { Type of } \\
\text { Facility }\end{array}$ & Facility & $\begin{array}{l}\text { Type of } \\
\text { Facility }\end{array}$ & Facility & $\begin{array}{l}\text { Type of } \\
\text { Facility }\end{array}$ \\
\hline $\begin{array}{l}\text { Gaibandha District } \\
\text { Hospital }\end{array}$ & CEmONC & $\begin{array}{l}\text { Kurigram District } \\
\text { Hospital }\end{array}$ & CEmONC & $\begin{array}{l}\text { Jamalpur District } \\
\text { Hospital }\end{array}$ & CEmONC \\
\hline Sundergonj UHC & CEmONC & Nageswari UHC & CEmONC & Islampur UHC & CEmONC \\
\hline Fulchari UHC & BEmONC & $\begin{array}{l}\text { Bhurungamari } \\
\text { UHC }\end{array}$ & BEmONC & Melandah UHC & BEmONC \\
\hline Saghata UHC & $\mathrm{BEmONC}$ & Chilmary UHC & $\mathrm{BEmONC}$ & Bakshiganj UHC & BEmONC \\
\hline
\end{tabular}

Note: Facilities in Jamalpur will not receive any fund for coupon. UHC- Upazila Health Complex; CEmONCComprehensive Emergency Obstetric and Newborn Care; and BEmONC-Basic Emergency Obstetric and Newborn Care.

\section{Agreement and contracting authorities}

According to the approved guidelines, the institution-based P4P and/or Coupon Committees (the Committees) will receive and utilize the fund of the P4P project. The Committees are composed of the six members (Tables 2 and 3), and chaired by the Civil Surgeons/Superintendents in the District Hospitals; and Upazila Health and Family Planning Officers in the UHCs.

Before receiving any fund, the Committees of the 12 facilities will sign agreements with the Council about receiving fund for making payment against incentives, coupons, and incurring expenses for drugs, consumables and maintenance of the facilities under the P4P project. The agreement will be signed by the Chairperson of the P4P and/or Coupon Committee and Head of the health facility while the Country Director of the Council will sign on behalf of the Council.

\section{Table 2. District Hospital Pay-for-Performance and/or Coupon Committee}
1. Civil Surgeon/Superintendant
Chairperson
2. Deputy Director-Family Planning/
Member Assistant Director (Clinical Contraception)
3. Nursing Supervisor
Member
4. An NGO representative (BRAC/CARE)
Member
5. A BMA representative
Member
6. Resident Medical Officer
Member-Secretary

2 A BEmONC facility (1) administer parenteral antibiotics; (2) administer uterotonic drugs (i.e., parenteral oxytocin); (3)
administer parenteral anticonvulsants for preeclampsia and eclampsia (i.e., magnesium sulfate). (4) manually remove the
placenta (5) remove retained products (e.g. manual vacuum extraction, dilation and curettage); (6) perform assisted
vaginal delivery (e.g. vacuum extraction, forceps delivery); and (7) perform basic neonatal resuscitation (e.g., with bag
and mask). A CEmONC facility additionally perform surgery (e.g., caesarean section); and blood transfusion. 
Table 3. Upazila Health Complex Pay-for-Performance and/or Coupon Committee

1. Upazila Health and Family Planning Officer

2. Upazila Family Planning Officer/ Assistant Upazila Family Planning Officer

3. Nursing Supervisor

4. An NGO representative (BRAC/CARE)

5. A representative from Upazila Parishad

6. Resident Medical Officer
Chairperson

Member

Member

Member

Member

Member-Secretary

The Committees will engage appropriate personnel from the facilities to pay cash or check to the facility-based and field-based beneficiaries, coupon patients and vendors, to distribute medicines, to keep the books of accounts and to prepare expenditure statements. The Committee and its designated personnel will follow the standard accounting practices, approved guidelines, and procedures put forth in the Financial Mechanism Manual.

The P4P and/or Coupon Committees will perform the following activities-

1. Open the P4P and/or Coupon Committee's bank account.

2. Align the facility-based MNCH care team and QATs for each quarter.

3. Sign an agreement with the Council about receiving fund for the P4P project and incur expenses following the standard accounting practices, approved guidelines and procedures put forth in the Financial Mechanism Manual.

4. Assess the projected expenses, and request monthly advance from the Council by submitting an advance request form. The advance request should be made in broad headings of (i) incentive payment to facility-based $\mathrm{MNCH}$ beneficiaries, (ii) incentive payment to field workers for coupon distribution and/or referral, (iii) incurring expenses against Drugs, Consumables and Maintenance fund, (iv) payment against transportation coupon to the coupon beneficiaries, (v) acquisition of medicines for distribution among the coupon beneficiaries, (vi) payment to the diagnostic centers and/or the GoB Treasury for providing diagnostic services to the coupon beneficiaries, and (vii) payment against incidental costs to the coupon beneficiaries. Upon receiving the advance request, the Council will arrange transfer of the fund to the P4P and/or Coupon Committee's bank account.

5. The CEmONC health facilities will identify qualified diagnostic centers that may be recommended for the patients with coupons to have emergency diagnostic services unavailable at the facility; and carry out contract with the diagnostic centers to reimburse them for providing services on monthly basis.

6. Approve of incentive distribution to providers/beneficiaries' bank accounts based on institutional performance, providers' presence, coupon distribution and referrals made by the field workers.

7. Approve of coupon distribution (in Gaibandha and Kurigram) according to the list of poor pregnant women and poor mothers of neonates and under-five children submitted by the respective Family Welfare Assistants (FWAs), Health Assistants (HAs) and NGO field workers after receiving certification from the Union Health and Family Planning Committees. 
8. Provide incentives by issuing Account Payee checks as performance incentives to the providers, diagnostic service facilities and vendors; and pay cash to the field workers and vendors.

9. Arrange for providing medicines, cash for transportation and incidental costs to coupon beneficiaries at the facilities.

10. Monitor financial reimbursement to the P4P scheme beneficiaries and expenses made for the DCM fund; and coupon beneficiaries.

11. Manage and keep appropriate records, vouchers and supporting documents of the facility beneficiaries and field workers' incentive, the transportation, medicine/diagnostic, and incidental coupons, payment to diagnostic facilities, and the DCM Funds.

12. Provide the lists of incentive and coupon beneficiaries, and diagnostic facilities certified by the head of the institution and validated by the P4P and/or Coupon committee while requesting advance fund from the Council.

13. Settle the advance monthly by submitting the expense account along with the original receipts and supporting documents against facility beneficiaries and field workers' incentive, coupon and DCM fund to the Council. Having one's name as a member of the MNCH team will not make anyone eligible for receiving incentive unless the facility satisfactorily meets the quantitative and qualitative targets. In order to receive the incentives, the beneficiaries will have to work at the facility during the measured period. Thus, the advance will be adjusted upon receiving the original receipts and certification of appropriateness of the books of accounts and supporting documents from the Audit Firm appointed by the Council.

14. Take measures to ensure smooth fund flow and prevent fraud and misappropriation of fund. For achieving this goal, the Committee will cooperate with the Council and its appointed Audit Firm in establishing and practicing sound financial mechanism with regard to incentive disbursement, coupon payment, medicine acquisition and distribution, payment for the prescribed diagnostic services and incurring expenses against DCM fund. In case of anomaly or fraudulent activities, the $\mathrm{P} 4 \mathrm{P}$ and/or Coupon Committee will take corrective measures immediately, as advised by the DGHS and Audit Firm.

15. Discuss with the facility-based QATs in utilizing DCM fund and other activities.

16. Meet at least once a month to review the performance of the facility and coupon distribution (only in Gaibandha and Kurigram), and take appropriate measures. If necessary, the P4P and/or Coupon Committee may meet more frequently. 


\section{E. Type of funds}

Depending on the implementation activities and type of infrastructure, the facilities will receive six types of fund. Facilities in all the districts will receive incentive and DCM funds. Facilities in Gaibandha and Kurigram districts, except for the District Hospitals, will receive funds for coupon while the District Hospitals and the CEmONC UHCs will receive fund to make payment to the diagnostic services to the coupon beneficiaries, which is not applicable to the BEmONC facilities.

1. Incentive fund:

- Incentive payment to the facility-based $\mathrm{MNCH}$ service providers, managers; administrative and support staff based on quarterly target achievement; and (12 facilities)

- Incentive payment to the field workers including Family Welfare Assistants, Health Assistants and NGO volunteer/workers for coupon distribution and/or referral services (6 UHCs of Gaibandha and Kurigram districts for coupon distribution; 9 UHCs for referral payments).

2. Drugs, Consumables and Maintenance and Other fund (12 facilities):

- Purchasing essential drugs for clients, and consumables that are required by the providers in providing EmONC services; and

- Incurring expenses for maintenance of the facilities and P4P activities.

3. Coupon fund for transportation costs (8 facilities of Gaibandha and Kurigram districts):

- Payment for transportation cost to coupon clients for receiving EmONC care.

4. Coupon fund for medicines (8 facilities of Gaibandha and Kurigram districts):

- Payment for purchasing medicines for coupon clients receiving EmONC care.

5. Coupon fund for incidental expenses (8 facilities of Gaibandha and Kurigram districts):

- Payment for incidental cost to coupon clients for receiving hospitalized EmONC care.

6. Coupon fund for diagnostic services:

- Payment to diagnostic service centers for providing approved diagnostic services to coupon clients (4 CEmONC facilities of Gaibandha and Kurigram districts).

- Payment to the GoB Treasury for providing diagnostic services from the facility-based pathological labs to the coupon clients (Gaibandha and Kurigram District Hospitals).

\section{F. Requisite documents for receiving funds in advance, incurring expenses, \& advance settlement}

According to the approved guidelines, the institution-based P4P and/or Coupon Committees will receive fund in advance from the Council based on monthly advance request made to the Council by the Committees. The fund request will be made along with necessary projection and supporting 
documents. Upon receiving the advance request and necessary documents, the Council will make arrangement to transfer the monthly advance amount to the $\mathrm{P} 4 \mathrm{P}$ and/or Coupon Committee's bank account. The Committee, after receiving the fund, will incur expenses following the guidelines. Within 15 days of the end of a month, the Committee will settle the advance by submitting an expense account with necessary vouchers and supporting documents. The Committee may make payments to the facility-based beneficiaries and field workers for incentives; to the coupon clients for transportation and incidental costs; to the diagnostic facilities and GoB Treasury for providing diagnostic services to the coupon clients; and for incurring expenses against the DCM Fund and acquiring medicines for the coupon clients. The Statement of Expenditure and all necessary vouchers and supporting documents will be certified by the Committee; which will then examined and certified by an Audit Firm engaged by the Council towards adjusting and settling the advance. A copy of the submitted documents will be retained by the Committee for at least four years.

Thus, receiving fund in the beginning of a month, incurring expenses over the month, and advance settlement at the end of a month involve several parties depending on the activity. The parties or the actors in financial transaction include the Council and the health facilities (i.e. the Cost Centers), facility-based pay-for-performance incentive recipients, field-based incentive recipients, coupon clients, diagnostic service centers, and vendors.

There are three major types of financial activities or transactions: (i) advance payment; (ii) advance settlement; and (iii) incurring P4P incentive, DCM, and coupon expenses. The first two types of activities will take place between the Council and the Committee in the beginning and in the end of a month, respectively, while the third type of activity will be initiated by the Committee with several parties over the month or in the end of a month before settling the advance with the Council. For instance, incentive payment to the field-based incentive recipients for coupon distribution and referrals will be made in the end of every month; incentive payment to the facility-based pay-forperformance incentive recipients will take place at the end of quarterly evaluation by the QAGs while payment of transportation costs to coupon clients and making expenses using the DCM fund will take place over the month.

\section{G. Transaction matrix}

A maximum of 16 types of financial activities or transactions will be carried out by the health facilities or the cost centers involving different parties requiring different documents. This can be briefly presented in a matrix called the transaction matrix for easy and comprehensive understanding. The Table 4 furnishes the transaction matrix with the list of financial transactions and required documents/forms/tools for each transaction between the parties. The transactions briefly include fund flow and settlement of funds between the Council and the Cost Centers; and also between the Cost Centers and different beneficiaries and recipients of the fund including coupon and incentive beneficiaries, diagnostic service centers, the GoB Treasury and vendors. Specimens of the relevant forms are attached in Annex 1. 
Table 4. Transaction matrix with the list of transactions and required documents for transaction between the parties

\section{Transaction 1 \\ Monthly advance received by the P4P \\ and/or Coupon Committee from the \\ Council.}

$1^{\text {st }}$ party: The Committee

- Form_1: Signed agreement between the P4P Committee and the Council.

- Form_2: Request for monthly advance to the Population Council in seven headings: (i) incentive payment to facility based $\mathrm{MNCH}$ beneficiaries, (ii) incentive payment to field workers for coupon distribution and/or referral, (iii) expenses against Drugs, Consumables and Maintenance and Other fund, (iv) payment against transportation coupon to the coupon beneficiaries, (v) payment against incidental costs to the coupon beneficiaries, (vi) acquisition of medicines for distribution among the coupon beneficiaries and (vii) payment to the diagnostic centers for providing diagnostic services to the coupon beneficiaries.

- Form_3: List of facility-based incentive beneficiaries, their basic salary, and quarterly incentive amount calculated based on the first and second level of target achievement; and certified documents of last month's basic salary of the beneficiaries.

- Form_4_A: List of field-based beneficiaries for coupon distribution and/or referrals for FWAs.

- Form_4_B: List of field-based beneficiaries for coupon distribution and/or referrals for HAs.

- Form_4_C: List of field-based beneficiaries for coupon distribution and/or referrals for

\section{$2^{\text {nd }}$ party: The Council}

- Form_1: Signed agreement between the P4P and/or Coupon Committee and the Council.

- Form_10: Letter/advice informing the Committee about transfer of fund to the Committee's bank account. 
NGO workers.

- Form_5: List of drugs and consumables to be purchased, and maintenance services to be acquired in a month.

- Form_6_A: List of coupon beneficiaries.

- Form_6_B: Projected transportation costs for coupon beneficiaries.

- Form_6_C: Projected incidental costs for coupon beneficiaries.

- Form_7: List of Medicines to be acquired for the coupon beneficiaries.

- Form_8: Meeting resolution of the Committee on selection of diagnostic service centers attached to the list of nominated centers that will be referred to the coupon beneficiaries (applicable only to District Hospitals and facility with CEmONC Services).

- Form_9_A: Contract signed between the Committee of the CEmONC facilities and the diagnostic service centers about reimbursing payment against diagnostic services provided to the coupon beneficiaries.

- Form_9_B: Projected diagnostic costs for coupon beneficiaries.

- Form_17_C: Bank book

- Form_22_C: Bank reconciliation statement

\section{Transaction 2}

Incentive payment to facility-based $\mathrm{MNCH}$ beneficiaries.

\section{Paying by Account Payee Check}

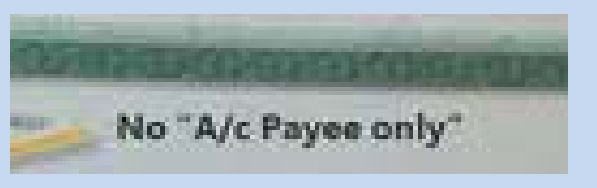

$1^{\text {st }}$ party: The Committee (Payment-maker)

- Form_11_A: Meeting resolution of the Committee on quarterly incentive amount distribution to the facility-based beneficiaries based on the Quality Assurance Group's performance $2^{\text {nd }}$ party: Facility-based beneficiaries

- Form_11_B: Facility-based beneficiaries' monthly attendance sheets attested by the facility head.

- Form_12: List of quarterly facility-based incentive amount distribution signed by the 
measurement report.

- Form_11_B: Facility-based beneficiaries' monthly attendance sheets attested by the facility head.

- Form_12: List of quarterly facility-based incentive amount distribution signed by the beneficiaries upon receiving the account payee checks.

- Form_13: Account payee checks issued in favor of the beneficiaries. Photocopies of the checks/Appropriately filled out check foils to be maintained.

- Form_17_B: Payment voucher (for each beneficiary)

- Form_17_C: Bank book

- Form_17_G: MNCH ledger

\section{Transaction 3}

Paying in Cash

Incentive payment to the field workers for coupon distribution and/or making successful referrals.

beneficiaries upon receiving the account payee checks.

- Form_13: Account payee check received. $1^{\text {st }}$ party: The Committee (Payment-maker)

- Cash payment.

- Form_14_A: Cash receipt for the field workers signed by both the beneficiaries and the facility head.

- Form_16_A: Referral slips distributed by the fieldworkers and presented by the patients signed by the service providers.

- Form_16_C: Payment register for referrals maintained by payment maker.

- Form_17_A: Petty cash register

- Form 17_B: Payment voucher:

- Form_17_D: Cash book

- Form_17_F: Field worker ledger $2^{\text {nd }}$ party: Field workers (FWAs, HAs, NGO workers)

- Form_19: Coupon Cards distributed by the field workers.

- Form_15_A: Coupon register maintained by the field workers.

- Form_15_B: Referral register maintained by the field workers.

- Form_16_A: Referral slips distributed by the fieldworkers and presented by the patients signed by the service providers.

- Form_14_A: Cash receipt for the field workers signed by both the beneficiaries and the facility head.

- Cash received. 


\section{Transaction 4 \\ Purchasing essential drugs and consumables and acquiring services for amount up to Taka 5,000.00 per purchase.}

$1^{\text {st }}$ party: The Committee (Payment-maker)

- Form_5: List of drugs, consumables and maintenance services to be incurred in a month.

- Cash payment

- Form_17_A: Petty cash register

- Form 17_B: Payment voucher

- Form_17_D: Cash book

- Form_17_E: DCM ledger

- Form_17_H: Stock register for DCM

\section{Transaction 5}

Purchasing essential drugs and consumables and acquiring maintenance services for amount above Taka 5,000.00 per purchase.

$1^{\text {st }}$ party: The Committee (Payment-maker)

- Form_5: List of drugs for providers, consumables and maintenance services to be incurred in a month signed by the Committee.

- Form_18: Comparison statement among minimum three (3) competitive quotations

- Form_13: Account payee check issued in favor of the vendors. Copy of account payee checks/Appropriately filled up check foils to be maintained.

- Form_17_B: Payment voucher

- Form_17_C: Bank book

- Form_17_E: DCM ledger

\section{$2^{\text {nd }}$ party: Vendor}

- Bill

- Money receipt

- Cash received

\section{Paying in Cash}

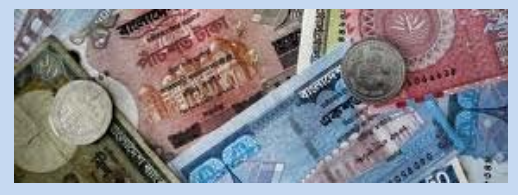

Paying by Account Payee Check

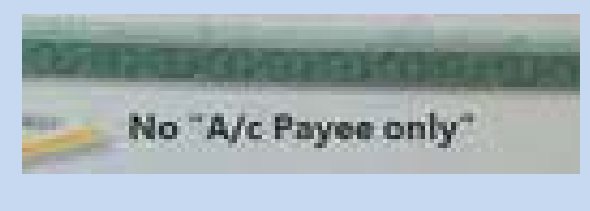

$2^{\text {nd }}$ party: Vendor

- Quotation

- Invoice

- Bill

- Money receipt

- Form_13: Account payee check received. 
- Form_17_H: Stock register for DCM.

\section{Transaction 6}

Distribution of essential drugs among the $\mathrm{MNCH}$ clients from DCM fund.

Giving out Medicines $1^{\text {st }}$ party: The Committee (Pharmacist)

- Form_20: Medicine receipt signed by the Pharmacist and approved by the UHFPO/RMO.

- Form 16_D: Medicine register for MNCH clients maintained by the Pharmacist.

- Form_17_H: Stock register for DCM.

- Medicine provided.

\section{Transaction 7}

Payment of transportation cost to coupon beneficiaries.

\section{$2^{\text {nd }}$ party: $\mathrm{MNCH}$ clients}

- Form_20: Medicine receipt signed/provided thumb impression upon receiving medicine.

- Medicine received. $1^{\text {st }}$ party: The Committee (Payment-maker)

- Cash payment

- Form_14_B: Cash Receipt for the coupon beneficiaries signed by the payment maker and approved by RMO/UHFPO.

- Form_16_B: Payment register for coupon beneficiaries maintained by Payment-maker.

- Form_17_A: Petty cash register

- Form 17_B: Payment voucher

- Form_17_D: Cash book

- Form_17_J: Transportation cost ledger.
Paying in Cash

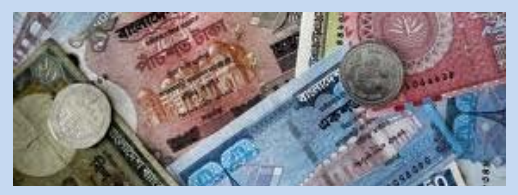

$2^{\text {nd }}$ party: Coupon beneficiaries

- Form_19: Coupon card signed by the service provider.

- Form_14_B: Cash Receipt for the coupon beneficiaries signed/provided thumb impression upon receiving cash. 


\begin{tabular}{|l|l|}
\hline Transaction 8 \\
Payment of the incidental costs to \\
coupon beneficiaries.
\end{tabular}




\begin{tabular}{|c|c|}
\hline $\begin{array}{l}\text { Transaction } 10 \\
\text { Payment for purchase of medicines for } \\
\text { coupon beneficiaries for amount above } \\
\text { Taka } 5,000.00 \text { per purchase. }\end{array}$ & $\begin{array}{c}\text { Paying by Account Payee Cbeck } \\
\text { No A/c Payse only" }\end{array}$ \\
\hline $\begin{array}{l}1^{\text {st }} \text { party: The Committee (Payment-maker) } \\
\text { - Form_7: List of medicines to be purchased } \\
\text { for the coupon beneficiaries in a month. } \\
\text { - Form_18: Comparison statement among } \\
\text { minimum three (3) competitive quotations } \\
\text { - Form_13: Account payee checks issued in } \\
\text { favor of the vendors. Copy of account } \\
\text { payee checks/Appropriately filled check } \\
\text { foils to be maintained. } \\
\text { - Form_17_B: Payment voucher } \\
\text { - Form_17_C: Bank book } \\
\text { - Form_17_K: Medicines for coupon } \\
\text { beneficiary ledger. } \\
\text { - Form_17_I: Stock register of Medicines for } \\
\text { coupon beneficiaries. }\end{array}$ & $\begin{array}{l}2^{\text {nd }} \text { party: Vendor } \\
\text { - Quotation } \\
\text { - Invoice } \\
\text { - Bill } \\
\text { - Money receipt } \\
\text { - Form_13: Account payee check received. }\end{array}$ \\
\hline $\begin{array}{l}\text { Transaction } 11 \\
\text { Distribution of medicines among the } \\
\text { coupon beneficiaries. }\end{array}$ & Giving out Medicines \\
\hline $\begin{array}{l}1^{\text {st }} \text { party: The Committee (Pharmacist) } \\
\text { - Form_20: Medicine receipt signed by the } \\
\text { Pharmacist and approved by the } \\
\text { UHFPO/RMO. } \\
\text { - Form 16_E: Medicine register for coupon } \\
\text { beneficiaries maintained by Pharmacist. } \\
\text { - Form_17_I: Stock register of Medicine for } \\
\text { coupon beneficiaries. } \\
\text { - Medicine provided. }\end{array}$ & $\begin{array}{l}2^{\text {nd }} \text { party: Coupon beneficiaries } \\
\text { - Form_19: Coupon card signed by the service } \\
\text { provider. } \\
\text { - Form_20: Medicine receipt Signed/provided } \\
\text { thumb impression upon receiving medicine. } \\
\text { - Medicine received. }\end{array}$ \\
\hline
\end{tabular}




\section{Transaction 12}

Providing diagnostic services to the coupon beneficiaries at the facility

\section{Providing diagnostic services}

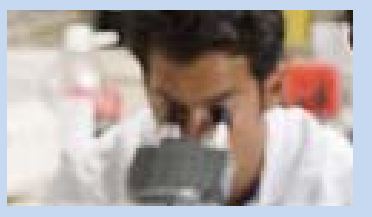

$1^{\text {st }}$ party: The Committee (Lab Technician)

- Form_21: Diagnostic receipt signed by the Lab Technician and approved by the UHFPO/RMO.

- Form 16_F: Diagnostic register for coupon beneficiaries maintained by Lab Technician.

- Diagnostic services provided.

\section{$\underline{2 \mathrm{n}}^{\text {nd }}$ party: Coupon beneficiaries}

- Form_19: Coupon card signed by the service provider and Lab Technician.

- Form_21: Diagnostic receipt signed by the Lab Technician and approved by the UHFPO/RMO.

- Diagnostic services received.

\section{Transaction 13}

Providing diagnostic services to the coupon beneficiaries from a

Diagnostic Test Center

\section{Providing diagnostic services}

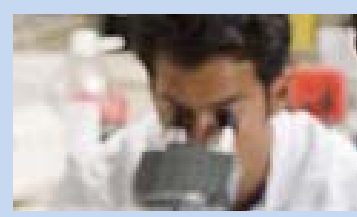

$1^{\text {st }}$ party: The Committee (Lab Technician)

- Form_21: Diagnostic receipt signed by the Lab Technician and approved by the UHFPO/RMO.

- Form 16_F: Diagnostic register for coupon beneficiaries maintained by Lab Technician.

- Diagnostic services provided. $2^{\text {nd }}$ party: Coupon beneficiaries

- Form_19: Coupon card signed by the service provider and Lab Technician.

- Form_21: Diagnostic receipt signed by the Lab Technician and approved by the UHFPO/RMO.

- Diagnostic services received.

\section{Transaction 14}

\section{Paying in Cash}

Payment to the GoB Treasury for providing diagnostic services from the facility labs to the coupon beneficiaries (Applicable only for the District Hospitals)

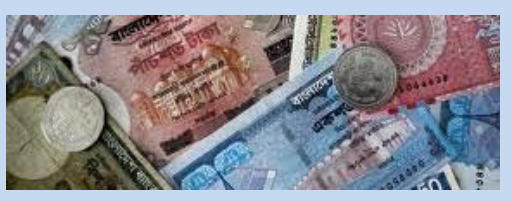

\section{$1^{\text {st }}$ party: The Committee}

- Form_21: Diagnostic service receipt signed/provided thumb impression by the coupon beneficiary and the facility lab technician

- Form_17_A: Petty cash register

\section{$2^{\text {nd }}$ party: GoB}

- GoB Treasury challan received 
- Form 17_B: Payment voucher

- Form_17_D: Cash book

- Form_17_N: Facility diagnostic cost ledger

- GoB Treasury challan purchased

\section{Transaction 15}

Payment to diagnostic service centers for providing prescribed diagnostic services to the coupon beneficiaries (Applicable only to District Hospitals and facilities with comprehensive EmONC services)

\section{$1^{\text {st }}$ party: The Committee}

- Form_9: Signed agreement between the Committee and the diagnostic centers.

- Form_21: Diagnostic service receipt signed/provided thumb impression by the coupon beneficiary and the authority of the diagnostic center.

- Form_13: Account payee checks issued in favor of the diagnostic center. Photocopies of the checks/ appropriately filled check foils to be maintained. If the own laboratory of the facility charges for diagnostic services then that amount will be paid through treasury chalan.

- Form_17_B: Payment voucher

- Form_17_C: Bank book

- Form_17_M: Diagnostic cost ledger.

\section{Transaction 16}

Settlement of advance amount with the Council

\section{$1^{\text {st }}$ party: The Committee}

- Form 17_B: Payment voucher with necessary vouchers and original documents

- Form_22_A: Monthly Expense Report

- Form_22_B: Check Outstanding Schedule

- Form_22_C: Bank reconciliation statement

- Form_22_D: Statement of Receipts and Payments

- Form_2: Request for advance for next month

\section{Paying by Account Payee Check}

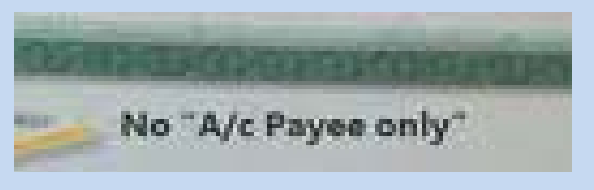

$2^{\text {nd }}$ party: Diagnostic service centers

- Form_9: Signed agreement between the Committee and the diagnostic centers.

- Form_19: Coupon card signed by the service provider of the facility and that of the diagnostic center.

- Form_21: Diagnostic service receipt signed/provided thumb impression by the coupon beneficiary upon receiving services.

- Form_13: Account payee check received.

\section{$2^{\text {nd }}$ party: The Council}

- Form_22: Adjust the advance against expenses for incentives, coupons and, DCM. Shortfall amount will be paid to the Committee while the excess advance amount will be adjusted against the next month's fund request.

- Form_10: Letter informing the Committee about the fund transfer to the Committee's bank account. 


\section{H. Conclusions}

The transaction matrix outlined in the Manual, the forms attached to the Appendix, and the four guidelines, hopefully, will guide and enable the Committee to initiate and carry out day-to-day financial activities to achieve the goal of the P4P project of creating an enabling system to reward the managers, providers, and support staff for their provide the best possible MNCH services in reducing the demand-side barriers by providing coupons to the poor women, newborns and underfive children. This may contribute to reduce maternal, neonatal and under-five children's morbidity and mortality in Bangladesh leading to achieving the Millennium Development Goals 4 and 5.

It is expected that all transactions will be transparent and carried out upholding the highest standard of moral and ethical values; which will automatically contribute to prevent any misappropriation and fraudulent activities. Both the Committees and the QATs will remain vigilant in preventing unfair utilization of funds. In case of any such activity, the Committee will take immediate remedial actions and secure the funds. The Committee, the QATs, and the Committee designated personnel who will carry out the financial activities and keep the books of accounts need complete and clear understanding of handling the financial matters following the Manual, and standard accounting practices and procedures. Such an understanding coupled with good intention will contribute to the successful implementation of the financial activities set forth in the pay-for-performance and coupon related incentive disbursement and DCM fund management.

The financial mechanism, in countries outside Bangladesh, is usually managed by Voucher Management Agencies while the public health facilities in Bangladesh, with technical assistance from the Council, will be carrying out this challenging task. Thus, it offers an alternative model of financial management mechanism. If successful, the financial mechanism of the P4P pilot project, as a model of the human resource innovation study, will set an example to the program managers, researchers and policy makers in the national, regional and international level in shaping the financial management of the future incentive-based initiatives. 


\section{References}

Directorate General of Health Services (DGHS), Population Council, and UNICEF. 2010. "Guidelines for introducing pay-for-performance (P4P) approach and subsidized coupons to increase utilization of maternal, newborn and child health services in Bangladesh." Dhaka, Bangladesh: DGHS, Population Council, and UNICEF.

Population Council. 2010. "Introducing pay-for-performance (P4P) approach and subsidized coupons to increase utilization of maternal, newborn and child health services in Bangladesh." Workshop Report. Dhaka, Bangladesh: Population Council.

Rahman, Laila, U. Rob, M.N. Talukder, I.A. Hena, F. Akter, M.A. Rahman, and M. Julkarnayeen. 2010. "Introducing pay-for-performance approach to improve maternal, newborn, and child health in Bangladesh.” P4P Research Update No. 1. Dhaka, Bangladesh: Population Council.

World Health Organization. 2009. Monitoring emergency obstetric care: A handbook. France: World Health Organization 


\title{
Appendix - 1
}

\section{Required documents and forms for financial transaction under the P4P Project}

\author{
Form 1: Sample Agreement between the Health Facilities and the Population Council
}

\author{
Agreement Between \\ The Population Council and \\ Kurigram District Hospital P4P and Coupon Committee
}

\subsection{Parties:}

This Agreement is signed between:

The Population Council, an international, nonprofit and nongovernmental organization having its office at House CES (B) 21, Road 118, Gulshan 2, Dhaka-1212, piloting the project, 'Introducing PayFor-Performance (P4P) Approach to Increase Utilization of Maternal, Newborn and Child Health Services in Bangladesh' to improve the maternal, newborn and under-five children services in Bangladesh. The study is under the operational plan of 2010-2011 of the Directorate General of Health Services (DGHS), Ministry of Health and Family Welfare of the Government of Bangladesh. The Population Council, hereinafter mentioned as the Council, presents itself as the FIRST PARTY.

\section{And}

Kurigram District Hospital P4P and Coupon Committee, a facility based committee has been formed to implement the P4P approach and coupon distribution. (P4P Committee formation Notice approved by the DGHS are attached herein Annexure 1). The P4P and Coupon Committee's activities have been outlined in the Guideline for the P4P and Coupon Committee, and approved by the DGHS (the Guideline and approval letter are attached herein Annexure 2). Kurigram District Hospital P4P and Coupon Committee, hereinafter mentioned as the Committee, presents itself as the SECOND PARTY.

\section{Terms and Scope of Work:}

The Committee will be responsible for setting benchmark and performance targets, managing incentive distribution, overseeing coupon distribution and its payment, and facilitating the P4P project activities. In addition, this committee will manage facility-based Drugs, Consumables and Maintenance (DCM) Fund. The committee's activities are guided by the approved Guidelines for Pay-for-Performance Incentive, and Coupon for Clients (Guidelines are attached herein Annexure3 and 4). The financial mechanism under the P4P project has been delineated in the Manual on Financial Mechanism for the Health Facilities (the Manual is attached herein Annexure- 5).

The detailed terms and scope of work/clauses for both parties are given below:

2.1 Following the Guidelines and the Manual, the second party will make monthly advance request to the first party against the following activities: 
(i) making incentive payment to facility based $\mathrm{MNCH}$ beneficiaries (once a quarter),

(ii) incurring expenses against Drugs, Consumables and Maintenance Fund,

(iii) making payment against transportation coupon to the coupon beneficiaries,

(iv) acquiring medicines for distribution among the coupon beneficiaries,

(v) making payment to the diagnostic centers for providing diagnostic services to the coupon beneficiaries, and

(vi) making payment against incidental costs to the coupon beneficiaries.

Upon receiving the advance request and necessary documents, the Council will make arrangement to transfer monthly advance amount to the Committee's bank account within seven (7) days.

2.2 The Committee will make transactions through the Committee's bank account by using joint signatures of the Committee Chairperson and Member Secretary. Fund operation and all disbursements have to be made in accordance and conformity with the guidelines set out in the Manual on Financial Mechanism for the Health Facilities, and the approved guidelines.

2.3 The Committee will make incentive payment to facility based $\mathrm{MNCH}$ beneficiaries, fieldworkers for coupon distribution and/or referral, expenses against Drugs, Consumables and Maintenance Fund, make payment against transportation and incidental coupon for coupon beneficiaries, acquire medicines and arrange for distribution of medicines among the coupon beneficiaries, and make payment to the diagnostic centers for providing diagnostic services to the coupon beneficiaries.

2.4 Within 15 days of the end of a month, the Committee will settle the advance by submitting a Statement of Expenditure, along with all original receipts, invoices and other related documents related to disbursements, to the Council. The Statement of Expenditure and all necessary vouchers and supporting documents will be certified by the Committee; which will then examined and certified by an Audit Firm engaged by the Council towards adjusting and settling the advance. A copy of submitted documents will be retained by the Committee.

2.5 Any unutilized or unspent amount will be adjusted with the next month's advance. Any unspent money after the project period will be returned to the Council.

2.6 The Committee is responsible individually and/or collectively for appropriate utilization of fund, settlement of the advances, and prevention of any misappropriation or fraudulent activities.

2.7 The Committee may assign any person(s) within the committee or within the facility to maintain or manage the multiple tasks of fund management.

2.8 The Committee will maintain all related documents, vouchers and books of accounts including bank book, cash book, ledgers along with a copy of all the documents submitted to the Council at the time of submission of monthly expenditure. These documents and records will be kept in the concerned facility office and/or at the office of the Committee 
Chairperson for a period of at least four (4) years after either the termination of this agreement or the completion of the P4P Project, whichever happens first.

2.9 The Committee will be responsible to appropriate use of funds and adjust the advanced amount and will cooperate with the internal and/or external audit teams.

2.10 For the project evaluation purpose, the Committee will keep necessary papers and documents for at least four (4) years after either the termination of this Agreement or the completion of the P4P Project, whichever happens first.

2.11 Any disagreement will be settled, firstly, in discussion between the two parties. The DGHS may mediate between the two parties, if needed.

\subsection{Duration of the Agreement:}

This Agreement will be effective from the date of signing the agreement up to the period of the P4P project completion.

4.0 This Agreement has been executed in two sets of original copies. In witness where of the parties have signed this Agreement in Dhaka or at the Respective Facility on the mentioned dates.

\section{Signed on behalf of:}

\section{The First Party}

Country Director

The Population Council

House CES(B) 21, Road 118

Gulshan 2, Dhaka-1212, Bangladesh.

Date:

Witnesses

1.

2.

\section{The Second Party}

Chairperson

Kurigram District Hospital

P4P and Coupon Committee

Kurigram, Bangaldesh.

\section{Date:}

Witnesses

1.

2. 


\section{Form-2}

\section{Introducing Pay-for-Performance (P4P) Approach to Increase Utilization of Maternal, Newborn and Child Health Services in Bangladesh}

Name of the Facility/ Cost Centre:

\section{Advance Request Form for the Month of}

Year:

\section{Date:}

To: Country Director

The Population Council, House CES (B) 21, Road 118 Gulshan, Dhaka, Bangladesh.

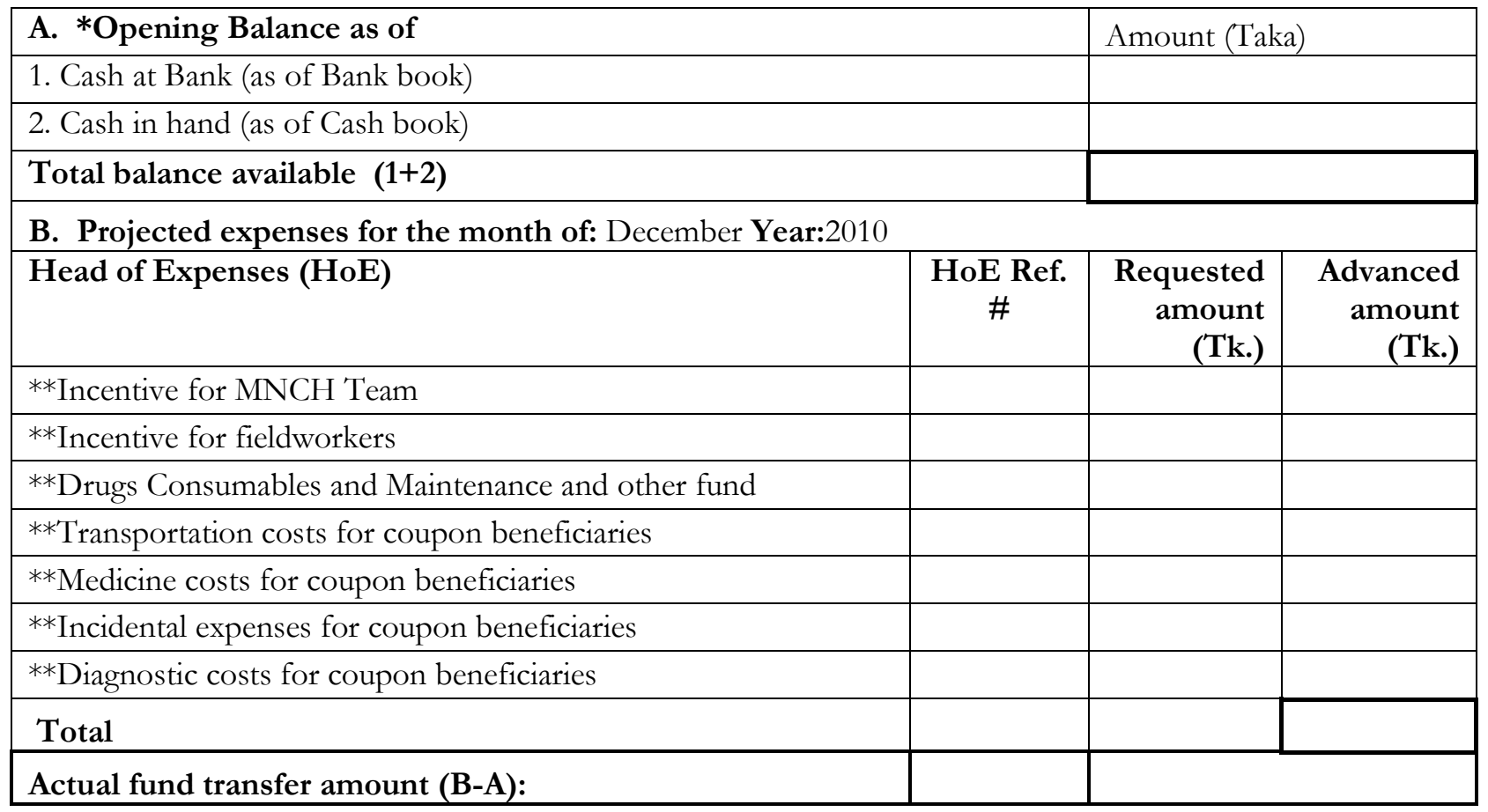

\section{Submitted by:}

Name:

Designation:

Date:
Reviewed by:

Name:

Designation:

Date:

\section{Approved by:}

Name:

Designation:

Enclosed: * i) Monthly bank statement should be attached along with bank reconciliation statement

* ii) Required documents as mentioned in the Manual 


\section{Form-3: Top Sheet}

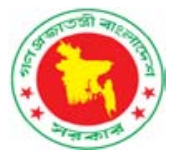

Introducing Pay-for-Performance (P4P) Approach to Increase Utilization of

Maternal, Newborn and Child Health Services in Bangladesh

List of Facility-based Incentive Beneficiaries and their Benefits

Name of the Facility/Cost Center:

Quarter:

Requested amount in Taka:

Certified By : (please tick) (CS/UHFPO)

Name:

Date:
Prepared By: (please tick) Head Assistant-cum-Accountant/Statistician/Cashier

Name:

Date:

*Enclosed: Certified office documents of the beneficiaries last month's basic salary. 
List of Facility-based Incentive Beneficiaries and their Benefits

Name of the Facility/Cost Center:

Quarter:

\begin{tabular}{|c|c|c|c|c|c|c|}
\hline Sl. No & Designation & Name of the incentive beneficiaries & $\begin{array}{l}\text { Benefit } \\
\text { ratio }\end{array}$ & $\begin{array}{l}\text { Basic salarty }{ }^{*} \\
\text { (Previous Month) }\end{array}$ & $\begin{array}{l}\text { Quarterly incentive } \\
\text { amount in Taka with } \\
\text { 1st level target } \\
\text { achievement }\end{array}$ & $\begin{array}{l}\text { Quarterly incentive } \\
\text { amount in Taka with } \\
2^{\text {nd level target }} \\
\text { achievement }\end{array}$ \\
\hline & & & & & & \\
\hline & & & & & & \\
\hline & & & & & & \\
\hline & & & & & & \\
\hline & & & & & & \\
\hline & & & & & & \\
\hline & & & & & & \\
\hline & & & & & & \\
\hline & & & & & & \\
\hline & & & & & & \\
\hline & & & & & & \\
\hline & & & & & & \\
\hline & & & & & & \\
\hline & & & & & & \\
\hline \multicolumn{5}{|c|}{ Total amount in Taka } & & \\
\hline
\end{tabular}


Introducing Pay-for-Performance (P4P) Approach to Increase Utilization of Maternal, Newborn and Child Health Services in Bangladesh

\section{Fund request for Field-based Beneficiaries}

\begin{tabular}{|r|r|}
\hline \multicolumn{2}{|c|}{ Total Fund Request for Field-based Beneficiaries } \\
\hline For FWA (Form-4_A)* & \\
\hline For HA (Form-4_B)* & \\
\hline For NGO (Form-4_C)* & \\
\hline Total requested amount in Taka & \\
\hline *Amount to be brought forward from Forms 4_A, 4_B and 4_C. \\
\hline
\end{tabular}

Certified By: (please tick) UHFPO/UFPO

Name:

Date:
Prepared By: (please tick) Head Assistant-cum-Accountant/Statistician/Cashier

Name:

Date: 


\section{Form- 4_A}

Introducing Pay-for-Performance (P4P) Approach to Increase Utilization of Maternal, Newborn and Child Health Services in Bangladesh

List of Family Welfare Assistant (FWA) for Coupon Distribution and/or Referrals

Name of the Facility/Cost Center: Month and Year:

\begin{tabular}{|l|l|l|l|}
\hline Total Number of FWA & $\begin{array}{c}\text { Total Projected number } \\
\text { of coupon distribution }\end{array}$ & $\begin{array}{c}\text { Total Projected number } \\
\text { of referral cases }\end{array}$ & $\begin{array}{c}\text { Total Requested } \\
\text { amount in Taka }\end{array}$ \\
\hline & & & \\
\hline
\end{tabular}

Certified By: UFPO

Name:

Date:
Prepared By: (please tick) Head Assistant-cum-Accountant/Statistician/Cashier

\section{Name:}

Date: 
List of Family Welfare Assistant (FWA) for Coupon Distribution and/or Referrals

Name of the Facility/Cost Center:

Month and Year:

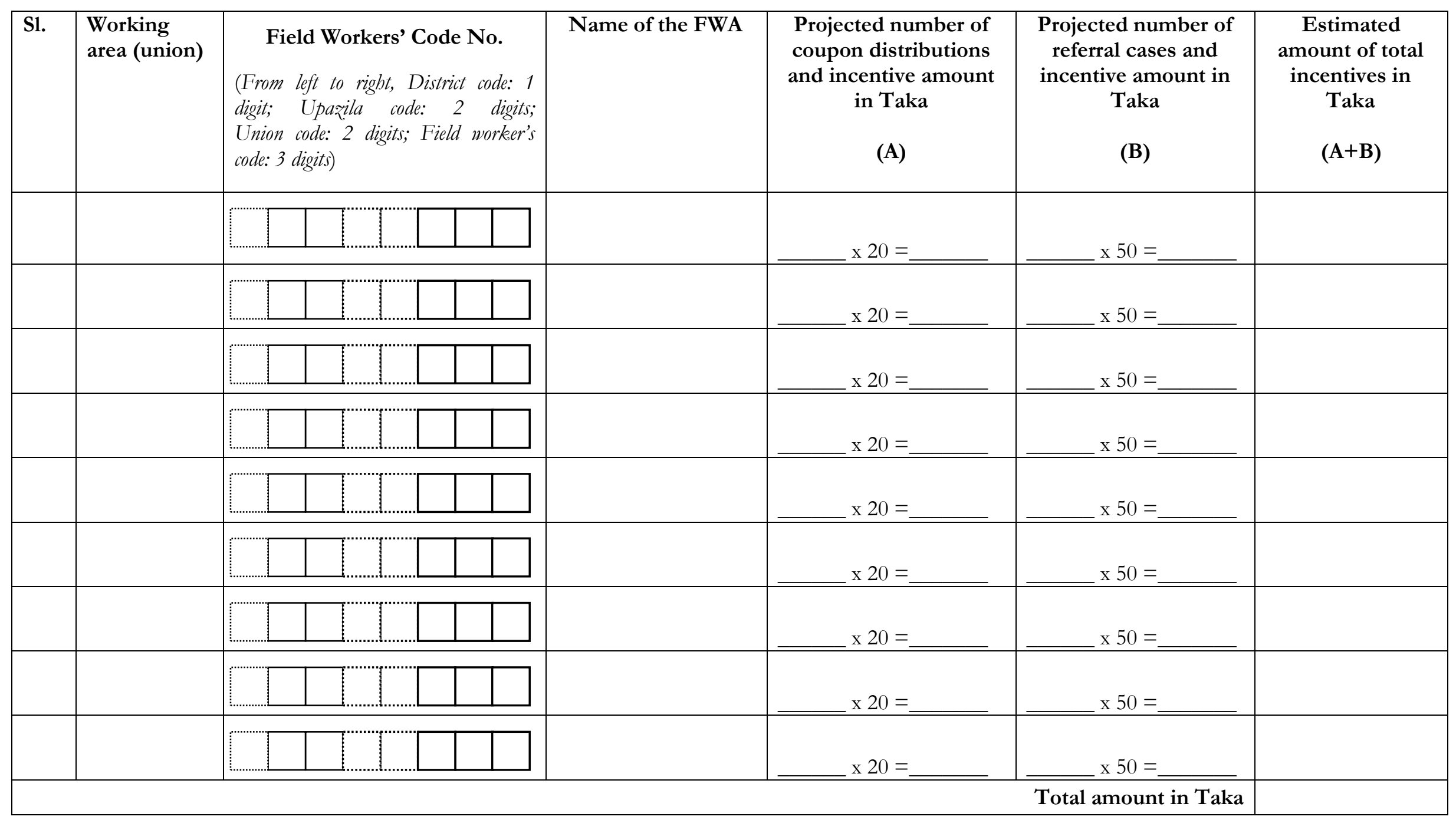


Form- 4_B

Introducing Pay-for-Performance (P4P) Approach to Increase Utilization of Maternal, Newborn and Child Health Services in Bangladesh

List of Health Assistant (HA) for Coupon Distribution and/or Referrals

Name of the Facility/Cost Center:

Month and Year:

\begin{tabular}{|l|c|c|c|}
\hline Total Number of HA & $\begin{array}{c}\text { Total Projected number } \\
\text { of coupon distribution }\end{array}$ & $\begin{array}{c}\text { Total Projected number } \\
\text { of referral cases }\end{array}$ & $\begin{array}{c}\text { Total Requested } \\
\text { amount in Taka }\end{array}$ \\
\hline & & & \\
\hline
\end{tabular}

Certified By: UHFPO

Name:

Date:
Prepared By: (please tick) Head Assistant-cum-Accountant/Statistician/Cashier

Name:

Date: 
List of Health Assistants (HAs) for Coupon Distribution and/or Referrals

Name of the Facility/Cost Center:

Month and Year:

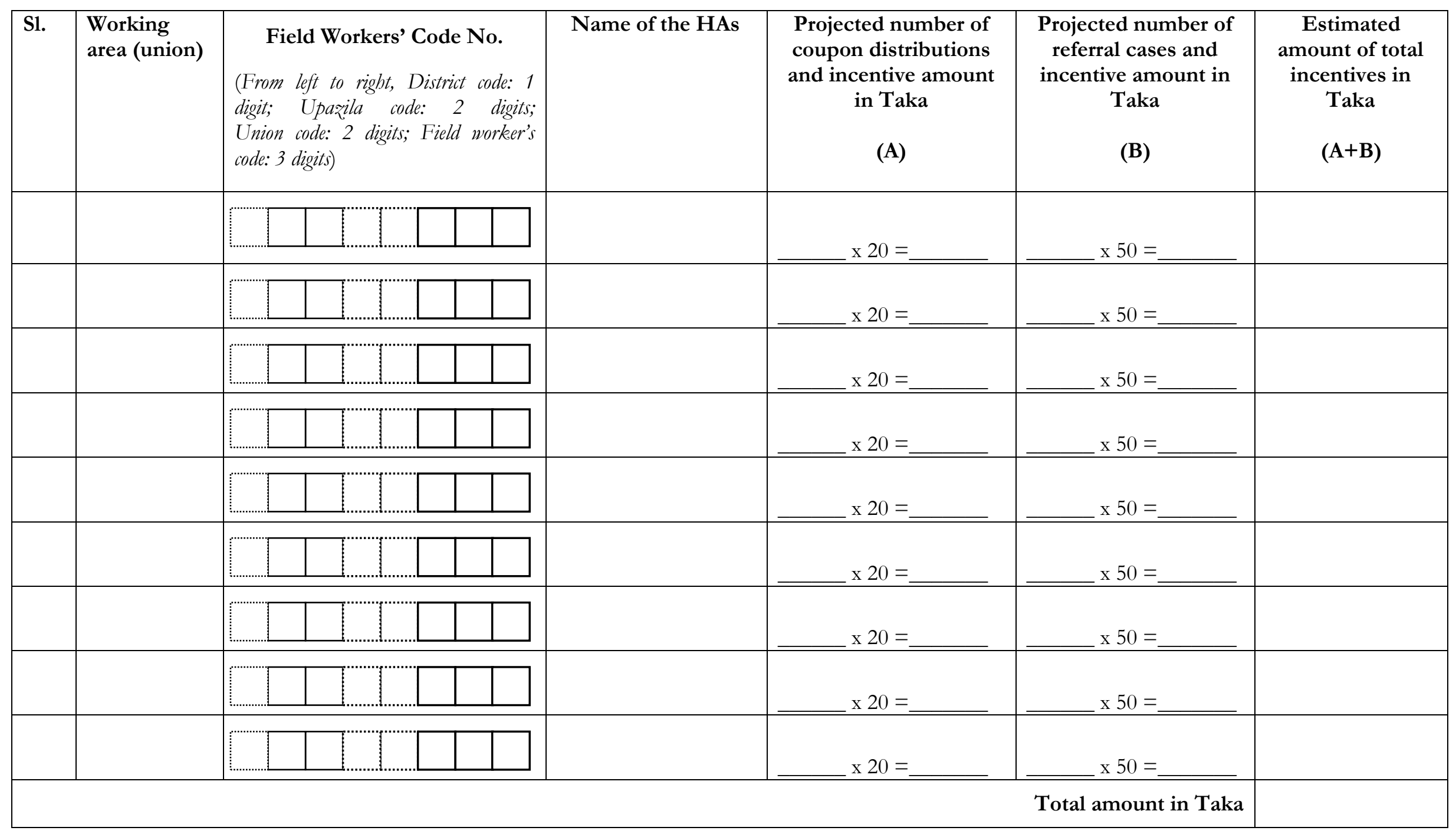


Form- 4_C Introducing Pay-for-Performance (P4P) Approach to Increase Utilization of Maternal, Newborn and Child Health Services in Bangladesh

List of NGO Workers/Volunteers for Coupon Distribution and/or Referrals

Name of the Facility/Cost Center:

\begin{tabular}{|c|c|c|c|}
\hline $\begin{array}{c}\text { Total Number of NGO } \\
\text { Workers/Volunteers }\end{array}$ & $\begin{array}{c}\text { Total Projected number } \\
\text { of coupon distributions }\end{array}$ & $\begin{array}{c}\text { Total Projected number } \\
\text { of referral cases }\end{array}$ & $\begin{array}{c}\text { Total Requested } \\
\text { amount on Money }\end{array}$ \\
\hline & & & \\
\hline
\end{tabular}

Certified By: (please tick) UHFPO/UFPO

Name:

Date:
Prepared By: (please tick) Head Assistant-cum-Accountant/Statistician/ Casbier

Name:

Date: 
List of NGO Worker/Volunteer for Coupon Distribution and/or Referrals

Name of the Facility/Cost Center:

Month and Year:

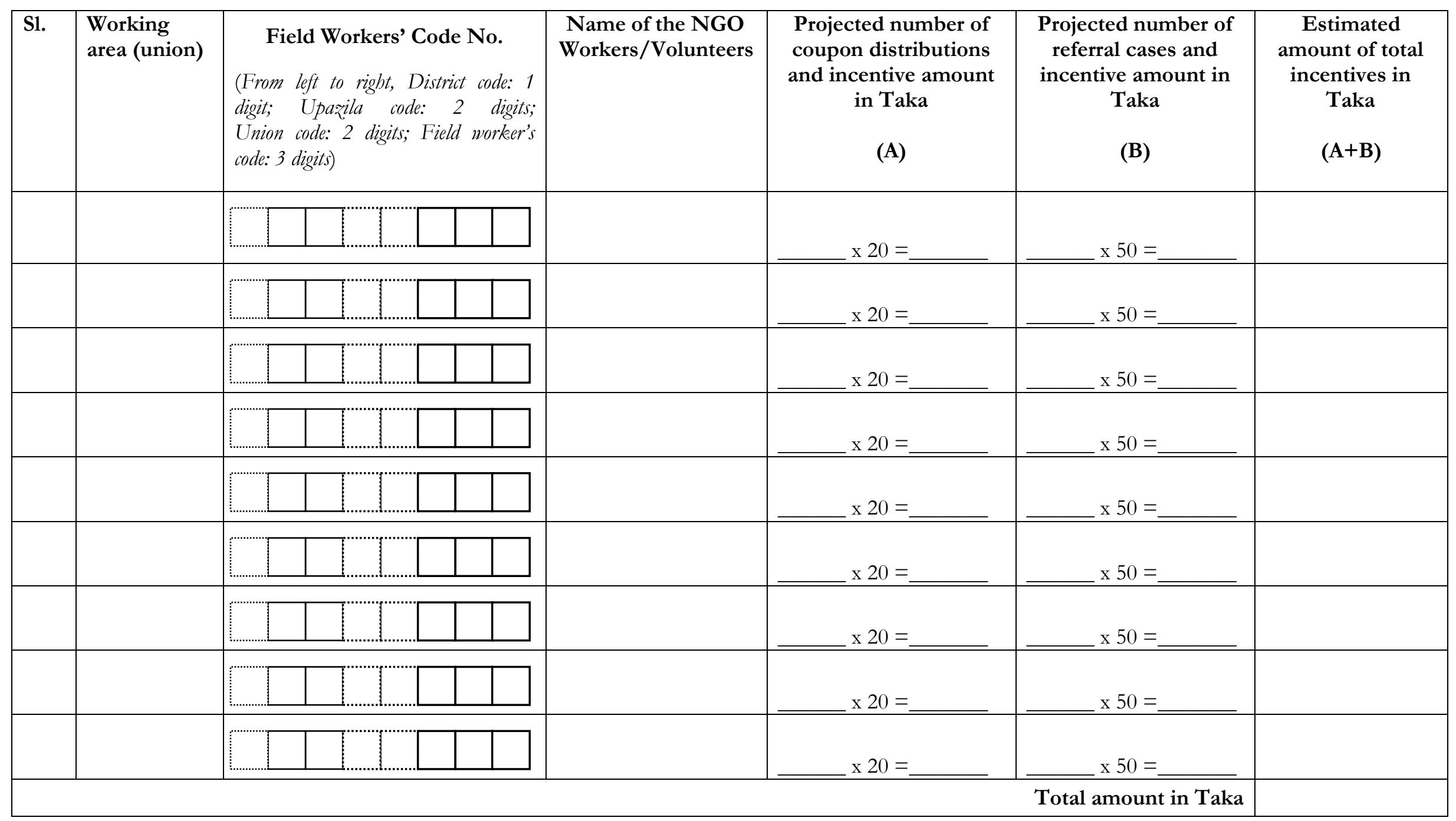


Form- 5: Top Sheet

Introducing Pay-for-Performance (P4P) Approach to Increase Utilization of Maternal, Newborn and Child Health Services in Bangladesh

\section{List of Drug, Consumables and Maintenance (DCM), and Others}

Name of the Facility:

Month and Year:

Total Requested amount in Taka:

Prepared By:

Name \& Designation:

Date:

Certified by: Gynae \& Obstetrician

Name:

Date:

Approved by: CS/UHFPO

Name:

Date:
Certified by: Pediatrician

Name:

Date:

Certified by: RMO

Name:

Date:

\section{Notes:}

* Drugs under the DCM fund include the drugs that are provided under the essential supply and drug (ESD) list, which the local authorities cannot buy in case of temporary shortage. These drugs are for all the MNCH clients.

**Consumables include the materials, equipments and other necessary items like hexisol, hand rub, gloves, which are necessary to provide quality $\mathrm{MNCH}$ care by the providers. These items are only used by the providers and the support staff. 
Form- 5

\section{List of Drug, Consumables and Maintenance (DCM), and Others}

Name of the Facility:

Month and Year:

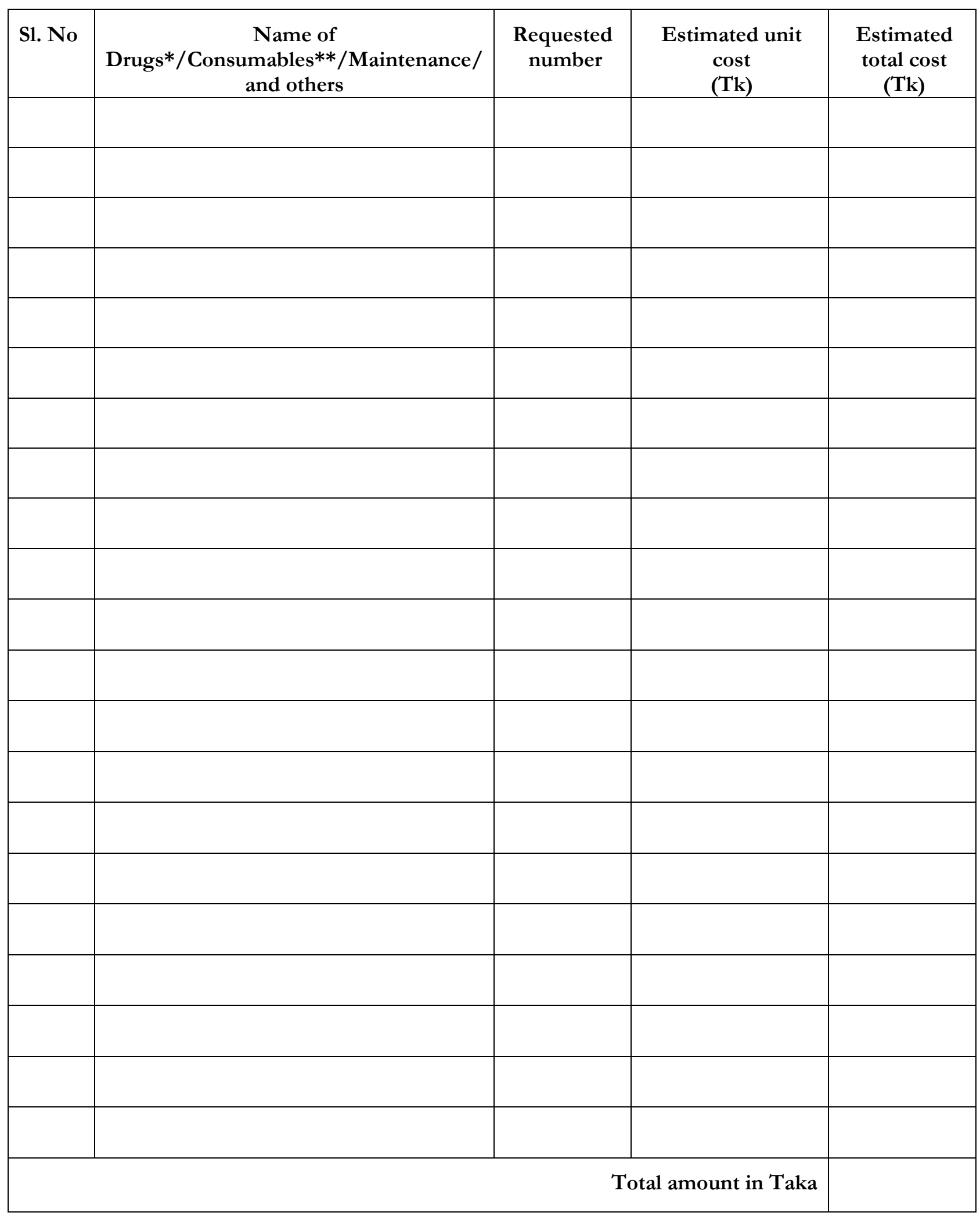


Form- 6_A Top sheet

Introducing Pay-for-Performance (P4P) Approach to Increase Utilization of

Maternal, Newborn and Child Health Services in Bangladesh

\section{List of Coupon Beneficiaries}

Name of the Facility:

Union:

Month and Year:

Total Number of Coupon Beneficiaries:

Assigned Fieldworker for Coupon Distribution

\begin{tabular}{|l|l|l|l|}
\hline Ward No. & HA & FWA & NGO worker \\
\hline & & & \\
\hline & & & \\
\hline & & & \\
\hline
\end{tabular}

Certified By: UHFPO

Name:

Date:
Prepared By: (please tick) Head Assistant-cum-Accountant/Statistician/Cashier Name:

Date: 
Form- 6_A

Name of the Facility:

\begin{tabular}{|l|l|l|l|}
\hline Sl. No & Name of the Coupon Beneficiaries & Name of Husband/Guardian & Address \\
\hline & & & \\
\hline & & & \\
\hline & & & \\
\hline & & & \\
\hline & & & \\
\hline & & & \\
\hline & & & \\
\hline & & & \\
\hline & & & \\
\hline & & Total Number of Coupon Beneficiaries: & \\
\hline & & &
\end{tabular}

\section{List of Coupon Beneficiaries}

Union:

Month and Year:

Name of Husband/Guardian 
Form- 6_B Top Sheet

Introducing Pay-for-Performance (P4P) Approach to Increase Utilization of Maternal, Newborn and Child Health Services in Bangladesh

Projected Transportation Cost for Coupon Beneficiaries

Total Requested amount in Taka:

Certified By: (please tick)CS/UHFPO/UFPO

Name:

Date:
Prepared By: (please tick) Head Assistant-cum-Accountant/Statistician/Cashier

Name:

Date: 


\begin{tabular}{|l|l|l|l|l|}
\hline S1. No & Name of the Union & $\begin{array}{c}\text { Estimated number of coupon } \\
\text { beneficiaries } \\
\text { (A) }\end{array}$ & $\begin{array}{c}\text { Average amount of } \\
\text { transportation cost in Taka } \\
\text { (B) }\end{array}$ & $\begin{array}{c}\text { Projected amount of } \\
\text { transportation cost in Taka } \\
\text { (A X B) }\end{array}$ \\
\hline & & & & \\
\hline & & & & \\
\hline & & & & \\
\hline & & & & \\
\hline & & & & \\
\hline & & & & \\
\hline & & & & \\
\hline & & & & \\
\hline
\end{tabular}




\section{Form- 6_C Top Sheet}

Introducing Pay-for-Performance (P4P) Approach to Increase Utilization of Maternal, Newborn and Child Health Services in Bangladesh

\section{Projected Incidental Cost of Coupon Beneficiaries}

Name of the Facility:

Month and Year:

Total Requested amount in Taka:

Certified By: (please tick) CS/UHFPO

Name:

Date:
Prepared By: (please tick) Head Assistant-cum-Accountant/Statistician/Cashier

\section{Name:}

Date: 
Form- 6_C

Projected Incidental Cost of Coupon Beneficiaries

Name of the Facility:

Month and Year:

\begin{tabular}{|c|c|c|c|c|c|}
\hline S1. No & Name of the Union & $\begin{array}{l}\text { Total number } \\
\text { of coupon } \\
\text { beneficiaries }\end{array}$ & $\begin{array}{c}\text { Projected number of } \\
\text { coupon beneficiaries who } \\
\text { may need hospitalization } \\
\text { (A) }\end{array}$ & $\begin{array}{c}\text { Average amount of } \\
\text { incidental costs in } \\
\text { Taka for } \\
\text { hospitalization (B) } \\
\end{array}$ & $\begin{array}{c}\begin{array}{c}\text { Projected amount of } \\
\text { incidental cost in Taka }\end{array} \\
(\text { A X B })\end{array}$ \\
\hline & & & & & \\
\hline & & & & & \\
\hline & & & & & \\
\hline & & & & & \\
\hline & & & & & \\
\hline & & & & & \\
\hline & & & & & \\
\hline & & & & & \\
\hline & & & & & \\
\hline & & & & & \\
\hline & & & & & \\
\hline & & & & & \\
\hline & & & & & \\
\hline & & & & Total amount in Taka & \\
\hline
\end{tabular}


Form- 7: Top Sheet

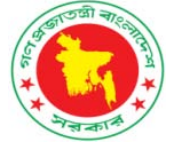

Introducing Pay-for-Performance (P4P) Approach to Increase Utilization of

Maternal, Newborn and Child Health Services in Bangladesh

\section{List of Medicines for Coupon Beneficiaries}

Name of the Facility:

Month and Year:

\section{Total Requested amount in Taka:}

Prepared By:

Name:

Date:

\section{Certified by: Gynae \& Obstetrician}

Name:

Date:

Approved by: CS/UHFPO

Name:

Date:
Certified by: Pediatrician

Name:

Date:

Certified by: RMO

Name:

Date:

Note: *Drugs for $\mathrm{MNCH}$ services, which are not available in the facility, will be purchased by using this fund for the coupon clients. 
Form- 7

\section{List of Medicines for Coupon Beneficiaries}

Name of the Facility:

Month and Year:

\begin{tabular}{|c|c|c|c|c|}
\hline S1. No & Name of Medicines* & $\begin{array}{l}\text { Requested } \\
\text { number }\end{array}$ & $\begin{array}{l}\text { Estimated unit } \\
\text { cost (Taka) }\end{array}$ & $\begin{array}{c}\text { Estimated total cost } \\
\text { (Taka) }\end{array}$ \\
\hline & & & & \\
\hline & & & & \\
\hline & & & & \\
\hline & & & & \\
\hline & & & & \\
\hline & & & & \\
\hline & & & & \\
\hline & & & & \\
\hline & & & & \\
\hline & & & & \\
\hline & & & & \\
\hline & & & & \\
\hline & & & & \\
\hline & & & & \\
\hline & & & & \\
\hline & & & & \\
\hline & & & & \\
\hline & & & & \\
\hline & & & & \\
\hline & & & & \\
\hline & & & & \\
\hline & & & & \\
\hline & & & otal cost in Taka & \\
\hline
\end{tabular}


Form- 8

Introducing Pay-for-Performance (P4P) Approach to Increase Utilization of

Maternal, Newborn and Child Health Services in Bangladesh

Meeting Minutes

\begin{tabular}{|c|c|}
\hline $\begin{array}{l}\text { Name of the } \\
\text { Committee: }\end{array}$ & Coupon Committee District Hospital/Upazila Health Complex P4P and \\
\hline \multicolumn{2}{|l|}{ Date: } \\
\hline \multicolumn{2}{|l|}{ Time: } \\
\hline \multicolumn{2}{|l|}{ Venue: } \\
\hline Agendum: & $\begin{array}{l}\text { Selection of the diagnostic test centers (DTCs) that the coupon beneficiaries will } \\
\text { be referred to for receiving diagnostic services, which are necessary, but } \\
\text { unavailable at the facility. }\end{array}$ \\
\hline \multicolumn{2}{|c|}{$\begin{array}{l}\text { Based on the DGHS approved Coupon for Clients Guideline for the P4P project, and in discussion } \\
\text { among the Committee members, the Committee has decided that total diagnostic test centers } \\
\text { (list attached) will be the referral diagnostic test centers for necessary diagnostic tests for the coupon } \\
\text { beneficiaries of the P4P project. The Committee has selected the test centers for having the ability to } \\
\text { provide the diagnostic tests services with high standard quality of care and accuracy. The Committee } \\
\text { will discuss and sign agreements with the nominated test centers about reimbursing payment against } \\
\text { diagnostic services provided to the coupon beneficiaries during the project period. }\end{array}$} \\
\hline
\end{tabular}

\section{Signature of the Committee Participants:}

\begin{tabular}{|c|c|c|c|}
\hline Sl. & Designation & Name & Signature and date \\
\hline 1. & & & \\
\hline 2. & & & \\
\hline 3. & & & \\
\hline 4. & & & \\
\hline 5. & & & \\
\hline 6. & & & \\
\hline 7. & & & \\
\hline 8. & & & \\
\hline
\end{tabular}


Form- 8

Attachment 1

List of the Diagnostic Test Centers selected for Referral to the Coupon Beneficiaries

\begin{tabular}{|c|c|c|c|}
\hline $\begin{array}{r}\text { Sl. } \\
\text { No }\end{array}$ & $\begin{array}{c}\text { Name and Address of the } \\
\text { Diagnostic Centers }\end{array}$ & $\begin{array}{c}\text { Name of the Owner of the } \\
\text { DTC with whom the } \\
\text { Agreement will be signed }\end{array}$ & $\begin{array}{c}\text { Specific services of the } \\
\text { DTC that could be used for the } \\
\text { coupon beneficiaries }\end{array}$ \\
\hline & & & \\
\hline & & & \\
& & & \\
& & &
\end{tabular}


Form- 9_A:

Agreement between

District Hospital/Upazila Health Complex P4P and/or Coupon

\title{
Committee and
}

\author{
Diagnostic Center
}

\subsection{Parties:}

This Agreement is signed between:

P4P and/or Coupon Committee, a facility based committee formed to implement the P4P approach and coupon distribution. (P4P Committee formation Notice approved by the DGHS are attached herein Annexure 1). P4P and/or Coupon Committee presents itself as the FIRST PARTY.

\section{And}

Diagnostic Center, a center selected by the respective P4P and/or Coupon Committee for providing selected pathological tests to the coupon holders. The selected diagnostic center presents itself as the SECOND PARTY.

\subsection{Terms and Scope of Work:}

Detailed terms and scope of work/clauses for both parties are given below:

2.1 The selected diagnostic center will provide selected pathological tests to a coupon holder upon receiving the referral slip from the coupon holder.

2.2 The center will provide the test to the patient after checking the slip and being satisfied that whether it is approved by the UH\&FPO or Resident Medical Officer (RMO) of the respective facility.

2.3 Diagnostic center will have the authority to provide two pathological tests to the coupon holders. These are: 1. Ultra-sonogram and 2. Blood Screening Test.

2.4 Costs of these tests will be decided mutually by both the P4P and/or Coupon Committee and the Diagnostic center.

2.5 Lab technician/service provider of the Diagnostic Center will keep a copy of referral slip from every client who received the service from the center and give a signed copy to the client after giving the service.

2.6 Diagnostic Center will be responsible to transmit the report of the diagnostic test result to the RMO of the respective District Hospital or Upazila Health Complex. 
2.7 At the end of the month, the diagnostic center will submit all the copies of referral slip to the P4P and/or Coupon Committee and will receive their money from the Coupon Payment Maker.

2.8 The RMO of the respective facility will examine the claim receipts submitted by the Diagnostic Center and advice the Coupon Payment Maker for payment.

2.9 The Diagnostic Center and the service provider/lab technician of the center should maintain the privacy of the client and will responsible be for the accuracy of the test.

\subsection{Duration of the Agreement:}

This Agreement will be effective from the date of signing the agreement up to the project completion.

4.0 This Agreement has been executed in two sets of original copies.

In witness where of the parties have signed this Agreement in Respective Facility/Diagnostic Center on the date mentioned 
Signed on behalf of:

\section{First Party}

$\mathrm{P} 4 \mathrm{P}$ and/or Coupon Committee

Chairperson

Witness

1.

2.
Second Party

Diagnostic Center

Authority of the Diagnostic Center

Witness

1.

2. 
Form- 9_B Top Sheet

Introducing Pay-for-Performance (P4P) Approach to Increase Utilization of Maternal, Newborn and Child Health Services in Bangladesh

Projected Diagnostic Test Cost for Coupon Beneficiaries from the Facility and the Diagnostic Test Centers

Name of the Facility:

Month and Year:

Total Requested amount in Taka:

Certified By: (please tick) CS/UHFPO

Prepared By: (please tick) Head Assistant-cum-Accountant/Statistician/Casbier)

Name:

Name:

Date:

Date: 


\begin{tabular}{|c|c|c|c|c|c|c|}
\hline S1. No & $\begin{array}{l}\text { Name of the } \\
\text { Upazila*/ Union }\end{array}$ & $\begin{array}{c}\text { Total number of } \\
\text { coupon beneficiaries }\end{array}$ & $\begin{array}{c}\text { Projected number of } \\
\text { coupon beneficiaries } \\
\text { referred to District } \\
\text { Hospital*/CEmONC } \\
\text { UHC }\end{array}$ & $\begin{array}{c}\text { Projected number of } \\
\text { coupon beneficiaries } \\
\text { who may need } \\
\text { diagnostic tests } \\
\text { (A) }\end{array}$ & $\begin{array}{c}\text { Average cost of } \\
\text { diagnostic test costs in } \\
\text { Taka } \\
\text { (B) }\end{array}$ & $\begin{array}{c}\text { Projected } \\
\text { amount of } \\
\text { diagnostic test } \\
\text { cost in Taka } \\
\text { (A X B) }\end{array}$ \\
\hline & & & & & & \\
\hline & & & & & & \\
\hline & & & & & & \\
\hline & & & & & & \\
\hline & & & & & & \\
\hline & & & & & & \\
\hline & & & & & & \\
\hline & & & & & & \\
\hline & & & & & & \\
\hline & & & & & & \\
\hline & & & & & 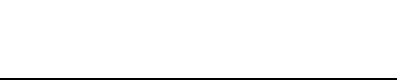 & \\
\hline \multicolumn{6}{|r|}{ Total amount in Taka } & \\
\hline
\end{tabular}

Note: For District Hospital "Name of the Upazila" is applicable in column 2 while for CEmONC UHC "Name of Union" will be applicable

*Column no. 4 is to be used only for District Hospital 
Form- 10 Introducing Pay-for-Performance (P4P) Approach to Increase Utilization of Maternal, Newborn and Child Health Services in Bangladesh

\section{Fund Transfer Advice}

The P4P and/or Coupon Committee Chair

Gaibandha/Kurigram/Jamalpur District Hospital/

Sundargonj/Saghata/Fulchari/Upazila Health Complex, Gaibandha/

Nageswari/Chilmary/Bhurungamari Upazila Health Complex, Kurigram/

Islampur/Melandah/Bakshiganj Upazila Health Complex, Jamalpur

Bangladesh.

\section{Re: Bank transfer in response to your fund request for the month of , year}

Dear Mr./Ms.

With reference to your fund request dated_ for Taka_, I would like to inform you that a Demand Draft No.__ of Taka___ dated___ has been issued in favor of the P4P and/or Coupon Committee Gaibandha/Kurigram/Jamalpur as an advance for the month of year__ under the pilot project on Introducing Pay-for-Performance (P4P) Approach to Increase Utilization of Maternal, Newborn and Child Health Services in Bangladesh.

We look forward to the Committee's fund settlement at the end of the month following the approved Guidelines, Financial Mechanism Manual and standard accounting practices and procedures.

Sincerely,

Country Director

Population council

House CES(B) 21, Road \# 118

Gulshan, Dhaka, Bangladesh. 
Form- 11

Introducing Pay-for-Performance (P4P) Approach to

Increase Utilization of Maternal, Newborn and Child Health Services in Bangladesh

\section{Meeting Minutes}

\begin{tabular}{|c|c|}
\hline Committee Name: & $\begin{array}{l}\text { District Hospital/ Upazila Health Complex P4P } \\
\text { and/or Coupon Committee }\end{array}$ \\
\hline \multicolumn{2}{|l|}{ Date: } \\
\hline \multicolumn{2}{|l|}{ Time: } \\
\hline \multicolumn{2}{|l|}{ Venue: } \\
\hline Agendum: & $\begin{array}{l}\text { Incentive amount distribution to the facility-based beneficiaries based on } \\
\text { the Quality Assurance Group's performance measurement report for the } \\
\text { quarter } \longrightarrow \text {, year: }\end{array}$ \\
\hline \multicolumn{2}{|c|}{ 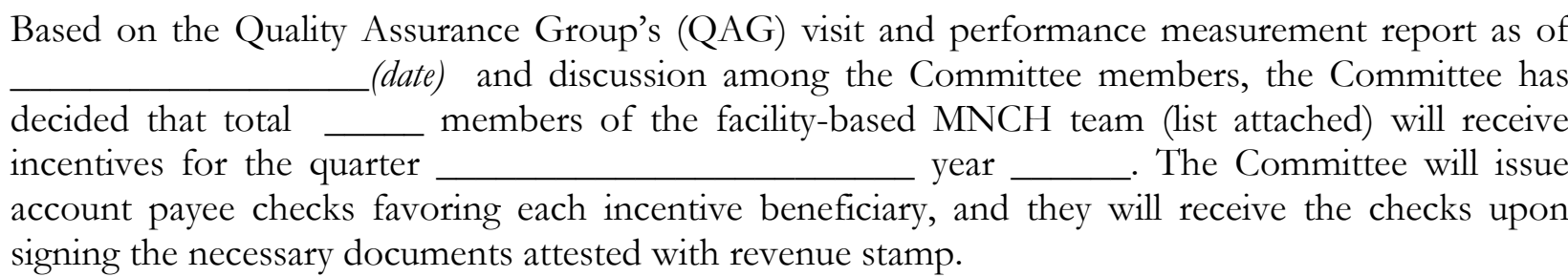 } \\
\hline
\end{tabular}

\section{Committee members' signature}

\begin{tabular}{|l|l|l|l|}
\hline Sl. & Designation & Name & Signature of the Members \\
\hline 1. & & & \\
3. & & & \\
4. & & & \\
5. & & & \\
\hline
\end{tabular}


Form- 11

Attachment 1

List of the selected MNCH Team Members as Incentive Beneficiaries and Incentive Amount For the Quarter Year

\begin{tabular}{|c|c|c|c|}
\hline Sl. & Designation & Name of the Incentive Beneficiaries & $\begin{array}{c}\text { Incentive Amount } \\
\text { (Taka) }\end{array}$ \\
\hline & & & \\
\hline & & & \\
\hline & & & \\
\hline & & & \\
\hline & & & \\
\hline & & & \\
\hline & & & \\
\hline & & & \\
\hline & & & \\
\hline & & & \\
\hline & & & \\
\hline & & & \\
\hline & & & \\
\hline & & & \\
\hline & & & \\
\hline
\end{tabular}


Form- 12: Top Sheet

Introducing Pay-for-Performance (P4P) Approach to Increase Utilization of Maternal, Newborn and

Child Health Services in Bangladesh

List of Facility-based Incentive Beneficiaries and their Benefits

Name of the Facility/Cost Center:

Quarter and Year:

Total Incentive amount Received by Beneficiaries: 
Name of the Facility/Cost Center:

Quarter and Year:

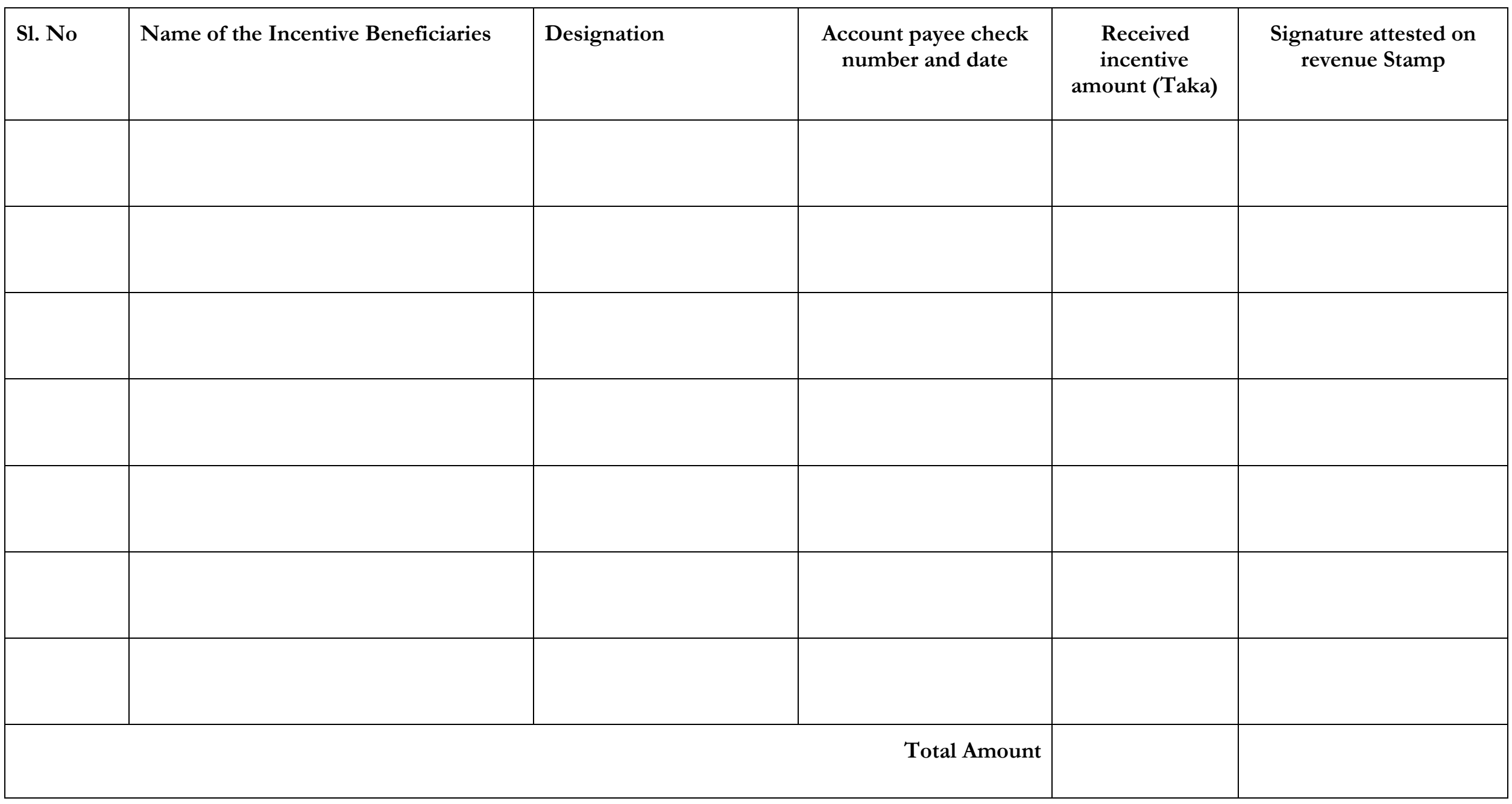


Name of the Facility:

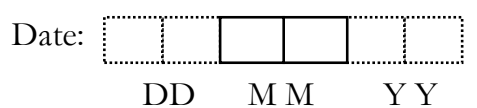

Sl. No.

Fieldworker's ID Number: \begin{tabular}{|l|l|l|l|l|}
\hline & & & & \\
\hline
\end{tabular}

(From left to right, District code: 1 digit; Uparila code: 2 digits; Union code: 2 digits; Fieldworker's code: 3 digits)

Name of Fieldworker:

Designation: HA/FWA/NGO worker

Area/Union: Month:

Year:

No. of Coupons distributed

Amount: \begin{tabular}{|l|l|l|l|}
\hline & & & \\
\hline
\end{tabular}

No. of Referrals:

Amount:

Total amount for distributed Coupons and Referrals:

\begin{tabular}{|l|l|l|l|}
\hline & & & \\
\hline
\end{tabular}

Name of Payment Maker: Signature Date:

Signature of Fieldworker: Date:

Approved by: (UHFPO/RMO/UFPO) Signature: Date:

\section{Form-14_A Population Council Copy}

\section{Cash Receipt for Fieldworkers}

(To be prepared by Payment Maker)

\section{Name of the Facility:}

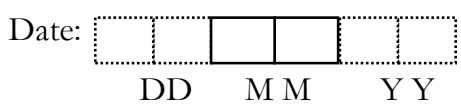

S1. No.

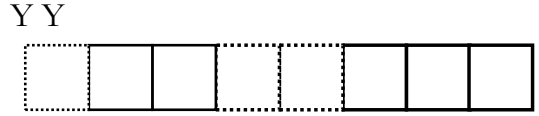

Fieldworker's ID Number:

(From left to right, District code: 1 digit; Upazila code: 2 digits; Union code: 2 digits; Fieldworker's code: 3 digits)

Name of Fieldworker:

Designation: HA/FWA/NGO worker
Area/Union:

Amount: Month:

Year:

No. of Coupons distributed

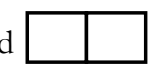

Total amount for distributed Coupons and Referrals:

Name of Payment Maker:

Signature

Date:

Signature of Fieldworker:

Date:

Approved by: (UHFPO/RMO/UFPO)

Signature:

Date: 
Name of the Facility:

Date:

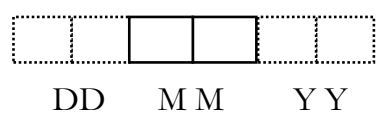

Sl. No.

DD $\quad$ M M Y Y

Fieldworker's ID Number:

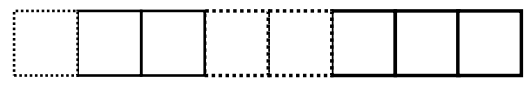

(From left to right, District code: 1 digit; Uparila code: 2 digits; Union code: 2 digits; Fieldworker's code: 3 digits)

Name of Fieldworker:

Designation: HA/FWA/NGO worker

Area/Union:

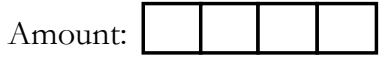

No. of Coupons distributed

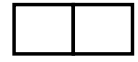

Total amount for distributed Coupons and Referrals:

Name of Payment Maker:

Signature

Date:

Signature of Fieldworker:

Date:

Approved by: (UHFPO/RMO/UFPO)

Signature:

Date: 


\section{Cash Receipt for Coupon Beneficiaries}

(To be prepared by Payment Maker)

Name of Facility:

Date: $: \square\left[\begin{array}{l|l|l|}\hline & \end{array}\right.$

S1. No.
DD M M Y Y

Coupon No: $\square$ :

(From left to right, District code: 1 digit; Upazila code: 2 digits; Union code: 2 digits; Coupon Distributor's code: 3 digits; Coupon Receiver's code: 4 digits)

Name: $\square$ Pregnant Women $\square$ Neonate $\square$ Under-5 child

Received Taka as $\square$ Transportation cost:

Incidental cost:

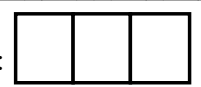

Total:

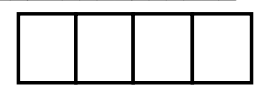

For receiving service:

Service Code:

Name Coupon Payment Maker:

Signature

Date:

Signature/fingerprint of Coupon Recipient:

Date:

Approved by: (UHFPO/RMO)

Signature:

Date:

Form 14_B Population Council Copy Cash Receipt for Coupon Beneficiaries

(To be prepared by Payment Maker)

Name of Facility:

Date:

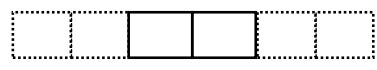

DD M M Y Y

Sl. No.

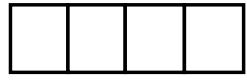

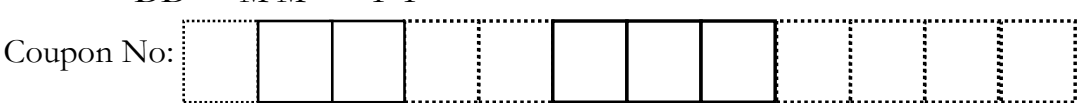

(From left to right, District code: 1 digit; Upazila code: 2 digits; Union code: 2 digits; Coupon Distributor's code: 3 digits; Coupon Receiver's code: 4 digits)

Name: $\square$ Pregnant Women $\square$ Neonate $\square$ Under-5 child

Received Taka as $\square$ Transportation cost:

Incidental cost:

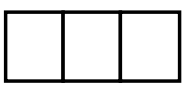

Total:

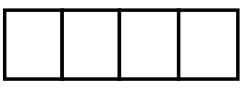

For receiving service:

Service Code:

Name Coupon Payment Maker:

Signature

Date:

Signature/fingerprint of Coupon Recipient:

Date:

Approved by: (UHFPO/RMO)

Signature:

Date: 


\section{Cash Receipt for Coupon Beneficiaries}

(To be prepared by Payment Maker)

Name of Facility:

Date:

Sl. No.

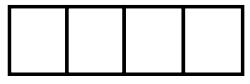

Coupon No:

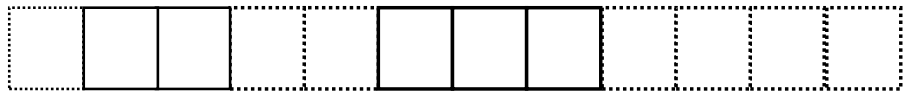

(From left to right, District code: 1 digit; Uparila code: 2 digits; Union code: 2 digits; Coupon Distributor's code: 3 digits; Coupon Receiver's code: 4 digits)

Name: $\square$ Pregnant Women $\square$ Neonate $\quad \square$ Under-5 child

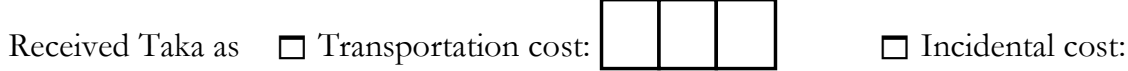

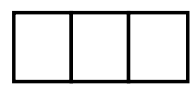

Total:

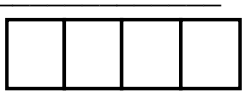

For receiving service:

Service Code:

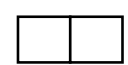

Name Coupon Payment Maker: Signature

Date:

Date:

Approved by: (UHFPO/RMO)

Signature:

Date: 
কুপন বিতরণকারীর নাম: কুপন বিতরণকারীর কোড নং:

\begin{tabular}{|l|l|l|l|l|l|l|l|l|l|l|l|l|l|}
\hline & & & & \\
\hline
\end{tabular}

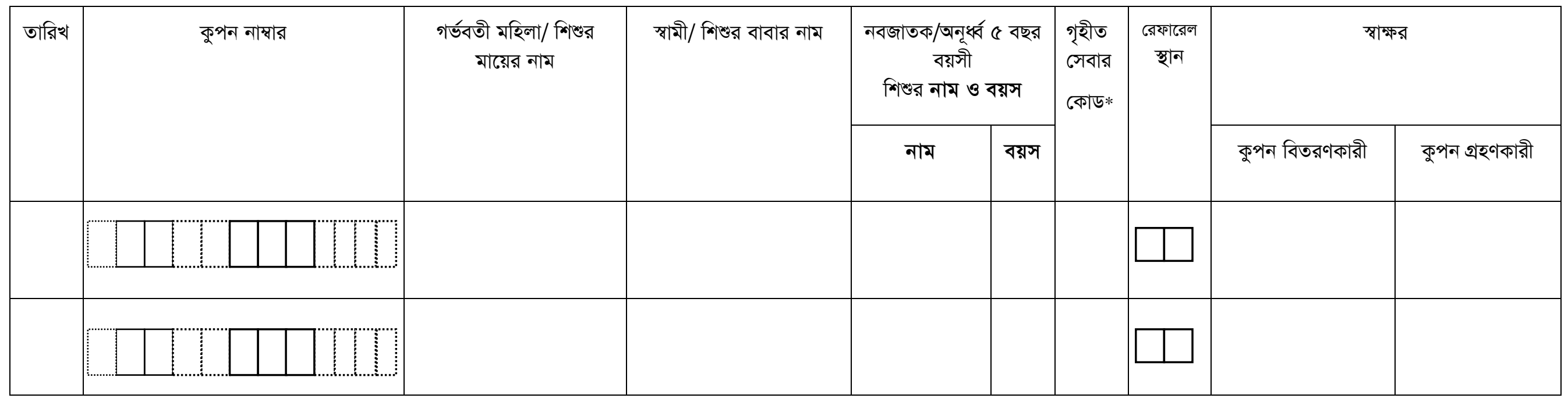

\section{* সেবার কোড একাধিক হতে পারে।}

কুপন বিতরণকারীর কোড নং (বাম থেকে ডানে): জেলা কোড: ১ সংখ্যা, উপজেলা কোড: ২ সংখ্যা, ইউনিয়ন কোড: ২ সংখ্যা, কুপন বিতরণকারী কোড: ৩ সংখ্যা । সেবার কোড: প্রসব পূর্ব ১ম সেবা=০১; প্রসব পূর্ব ২য় সেবা=০২; প্রসব পূর্ব ৩য় সেবা=০৩; প্রসবপূর্ব ৪র্থ সেবা=০৪; গর্ভকালীন জটিলতা ব্যবস্থাপনা =০৫; প্রসবকালীন সেবা=০৬; প্রসব পরবর্তী জটিলতা ব্যবস্থাপনা =০৭; প্রসব পরবর্তী জটিলতা ব্যবস্থাপনার ২য় সাক্ষাৎ =০৮; নবজাতকের জটিলতা ব্যবস্থাপনা =০৯; নবজাতকের জটিলতা ব্যবস্থাপনার ২য় সাক্ষাৎ=১০; অনূর্ধ্ব ৫ বছর বয়সী শিঙ্ডর জটিলতা ব্যবস্থাপনা =১১; অনূর্ব্ব ৫ বছর বয়সী শিশ্ডর জটিলতা ব্যবস্থাপনার ২য় সাক্ষাৎ =১২।

রেফারেল স্থানঃ ফুলছড়ি উপজেলা স্বসস্থ্য কমপ্লেক্স =০১; সাঘাটা উপজেলা স্বাস্থ্য কমপ্লেক্স =০২; সুন্দরগঞ্জ উপজেলা স্বাস্থ্য কমপ্লেক্স =০৩; গাইবান্ধা জেলা হাসপাতাল =০৪ ভুরুঙ্গামারী উপজেলা স্বাস্থ্য কমপ্লেক্স =০৫; চিলমারী উপজেলা স্বাস্থ্য কমপ্লেক্স =০৬; নাগেশ্বরী উপজেলা স্বাস্থ্য কমপ্লেক্স =০৭; কুড়িগ্রাম জেলা হাসপাতাল =০৮ । 
কুপন বিতরণকারীর রেজিষ্টার

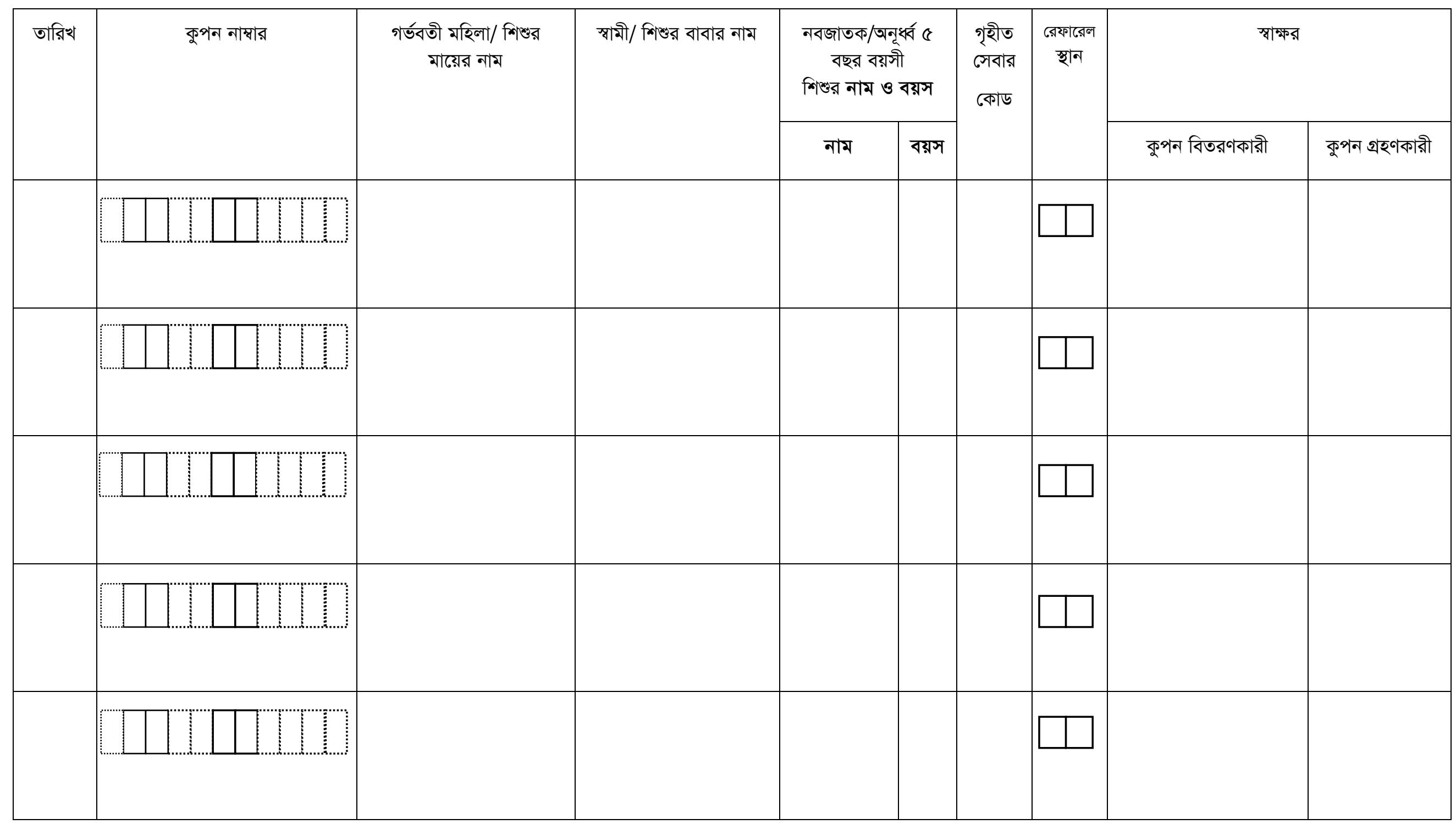


রেফারকারীর নাম:

রেফারকারীর কোড নং:

রেফারকারীর পদবী: HA /FWA/NGO কর্মী জেলা:গাইবান্ধা/কুড়িগ্রাম/জামালপুর; উপজেলা:

\begin{tabular}{|c|c|c|c|c|c|c|c|c|c|}
\hline \multirow[t]{2}{*}{ তারিখ } & \multirow[t]{2}{*}{$\begin{array}{l}\text { রোগী সনাক্তকরণ নাম্বারের } \\
\text { লেবা গ্রহণকারীর কোড সংখ্যা }\end{array}$} & \multirow[t]{2}{*}{$\begin{array}{l}\text { গর্ভবতী মহিলা/ শিশুর } \\
\text { মায়ের নাম }\end{array}$} & \multirow[t]{2}{*}{ স্বামী/ শিশ্ডর বাবার নাম } & \multicolumn{2}{|c|}{$\begin{array}{l}\text { অনূর্ধ্ব ৫ বছর বয়সী } \\
\text { শিশ্ডর নাম ও বয়স }\end{array}$} & \multirow{2}{*}{$\begin{array}{l}\text { রেফারেল } \\
\text { লেবার } \\
\text { কোড }\end{array}$} & \multirow[t]{2}{*}{$\begin{array}{l}\text { রেফারেল } \\
\text { স্থান }\end{array}$} & \multicolumn{2}{|l|}{ স্বাক্ষর } \\
\hline & & & & নাম & বয়স & & & রেফারকারী & সেবা গ্রহণকারী \\
\hline & & & & & & & & & \\
\hline & & & & & & & & & \\
\hline & & & & & & & & & \\
\hline & & & & & & & & & \\
\hline & & & & & & & & & \\
\hline
\end{tabular}

রোগী সনাক্তকরণ নাম্বার: (বাম থেকে ডানে): জেলা কোড: ১ সংখ্যা, উপজেলা কোড: ২ সংখ্যা, ইউনিয়ন কোড: ২ সংখ্যা, মাঠকর্মীর কোড: ৩ সংখ্যা, লেবা গ্রহণকারীর কোড: ৪ সংখ্যা ।

রেফারকারীর কোড নং (বাম থেকে ডানে): জেলা কোড: ১ সংখ্যা, উপজেলা কোড: ২ সংখ্যা, ইউনিয়ন কোড: ২ সংখ্যা, কুপন বিতরণকারী/ মাঠকর্মীর কোড: ৩ সংখ্যা ।

রেফারেল লেবার কোড: গর্ভকালীন ঝুকিপূর্ণ সমস্যা=১; প্রসব=২; ঝুঁকিপূর্ণ প্রসব=৩; প্রসব পরবর্তী ঝুঁকিপূর্ণ সমস্যা=8; নবজাতকের ঝুঁকিপূর্ণ সমস্যা=৫; অনূর্ব্ব ৫ বছর বয়সী শিশ্রর ঝুঁকিপূর্ণ সমস্যা=৬; রেফারেল স্থান: ফুলছড়ি উপজেলা স্বাস্থ্য কমপ্লেক্স =০১; সাঘাটা উপজেলা স্বাস্থ্য কমপ্লেক্স =০২; সুন্দরগঞ্ঞ উপজেলা স্বাস্থ্য কমপ্লেক্স =০৩; গাইবান্ধা জেলা হাসপাতাল =০৪ ভুরুঞ্গামারী উপজেলা স্বাস্থ্য কমপ্লেক্স =০৫; চিলমারী উপজেলা স্বাস্থ্য কমপ্লেক্স =০৬; নাগেশ্বরী উপজেলা স্বাস্থ্য কমপ্লেক্স =০৭; কুড়িগ্রাম জেলা হাসপাতাল =০৮বকশীগঞ্জ উপজেলা স্বাস্থ্য কমপ্লেক্স =০৯; ইসলামপুর উপজেলা স্বাস্থ্য কমপ্লেক্স =১০; মেলান্দহ উপজেলা স্বাস্থ্য কমপ্লেক্স =১১; জামালপুর জেলা হাসপাতাল =১২। 
Form-15_B

রেফারকারীর রেজিষ্টার

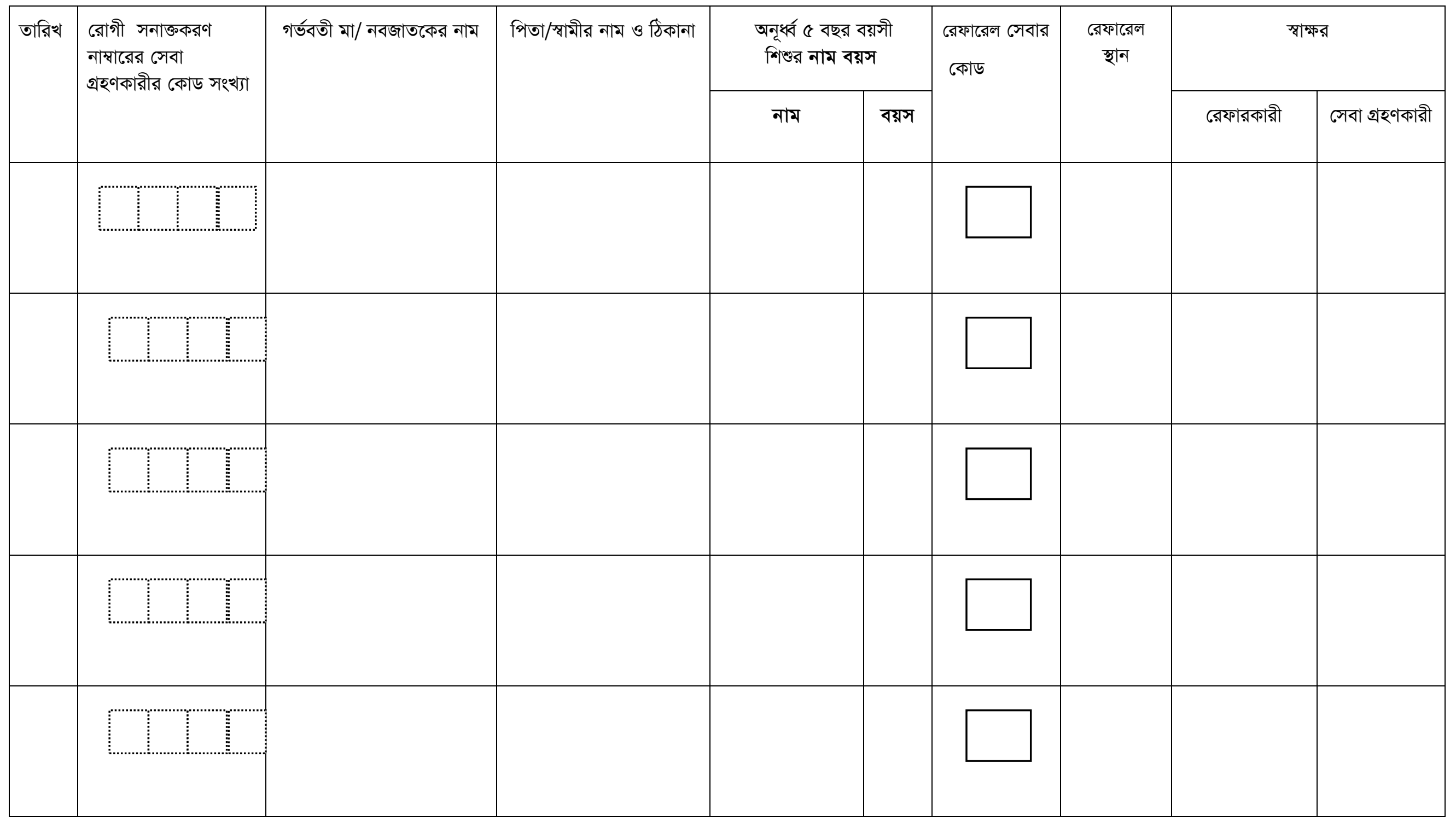




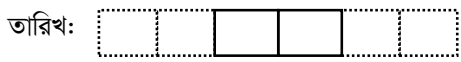

ক্রমিক নং:

কুপন নং/রোগী সনাক্তকরণ নাম্বার:

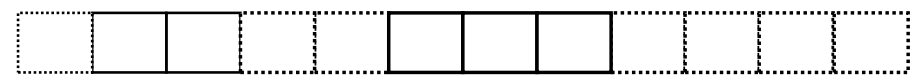

(বাম থেকে ডানে; জেলা কোড: ১ সংখ্যা, উপজেলা কোড: ২ সংখ্যা, ইউনিয়ন কোড: ২ সংখ্যা, মাঠকর্মীর কোড: ৩ সংখ্যা, সেবা গ্রহণকারীর কোড: 8 সংখ্যা)

সেবা গ্রহণকারীর নাম: $\square$ গর্ভবতী মা $\square$ নবজাতক $\square$ অনূর্ব্ব ৫ বছর বয়সী শিফ:

রেফারেল সেবার কোড:

রেফারেল সেবার কোড: গর্ভকালীন ঝুকিপূর্ণ সমস্যা=১; প্রসব=২; ঝুঁকিপূর্ণ প্রসব=৩; প্রসব পরবর্তী ঝুঁকিপূর্ণ সমস্যা=8; নবজাতকের ঝুঁকিপূর্ণ সমস্যা=৫; অনূর্ধব ৫ বছর বয়সী শিশ্তর ঝুঁকিপূর্ণ সমস্যা=৬। রেফারকৃত স্বাস্থ্যকেন্দ্র:

রেফারকৃত স্বাস্থ্যকেন্দ্রের কোড

মাঠকর্মীর নাম: মাঠকর্মীর পদবী: HA/FWA/NGO কর্মী

মাঠকর্মীর স্বাক্ষর : তারিখ:

সেবা প্রদানকারীর স্বাক্ষর:

তারিখ:

Form-16_A পপুলেশন কাউন্সিলের কপি

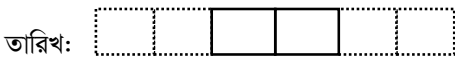

কুপন নং/রোগী সনাক্তকরণ নাম্বার:

\section{রেফারেল স্লিপ}

(মাঠকর্মী দ্বারা পূরণকৃত)

(বাম থেকে ডানে; জেলা কোড: ১ সংখ্যা, উপজেলা কোড: ২ সংখ্যা, ইউনিয়ন কোড: ২ সংখ্যা, মাঠকর্মীর কোড: ৩ সংখ্যা, সেবা গ্রহণকারীর কোড: 8 সংখ্যা)

সেবা গ্রহণকারীর নাম: $\square$ গর্ভবতী মা $\square$ নবজাতক $\square$ অনূর্ব্ব ৫ বছর বয়সী শিশ:

রেফারেল সেবার কোড:

রেফারেল সেবার কোড: গর্ভকালীন ঝুকিপূর্ণ সমস্যা=১; প্রসব=২; ঝুঁকিপূর্ণ প্রসব=৩; প্রসব পরবর্তী ঝুঁকিপূর্ণ সমস্যা=8; নবজাতকের ঝুঁকিপূর্ণ সমস্যা=৫; অনূর্ধ ৫ বছর বয়সী শিশ্তর ঝুঁকিপূর্ণ সমস্যা=৬। রেফারকৃত স্বাস্থ্যকেন্দ্র: রেফারকৃত স্বাস্থ্যকেন্দ্রের কোড

মাঠকর্মীর নাম: মাঠকর্মীর পদবী: HA/FWA/NGO কর্মী

মাঠকর্মীর স্বাক্ষর : তারিখ:

সেবা প্রদানকারীর স্বাক্ষর: তারিখ: 


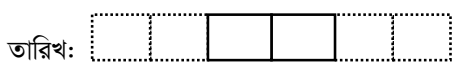

কুপন নং/রোগী সনাক্তকরণ নাম্বার:

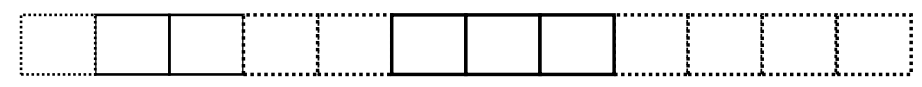

(বাম থেকে ডানে; জেলা কোড: ১ সংখ্যা, উপজেলা কোড: ২ সংখ্যা, ইউনিয়ন কোড: ২ সংখ্যা, মাঠকর্মীর কোড: ৩ সংখ্যা, সেবা গ্রহণকারীর কোড: 8 সংখ্যা) সেবা গ্রহণকারীর নাম: $\square$ গর্ভবতী মা $\square$ নবজাতক $\square$ অনূর্ব্ব ৫ বছর বয়সী শিশ্ট:

রেফারেল সেবার কোড:

রেফারেল সেবার কোড: গর্ভকালীন ঝুকিপূর্ণ সমস্যা=১; প্রসব=২; ঝুঁকিপূর্ণ প্রসব=৩; প্রসব পরবর্তী ঝুঁকিপূর্ণ সমস্যা=8; নবজাতকের ঝুঁকিপূর্ণ সমস্যা=৫; অনূর্ধর ৫ বছর বয়সী শিশ্তর ঝুঁকিপূর্ণ সমস্যা=৬। রেফারকৃত স্বাস্থ্যকেন্দ্র: রেফারকৃত স্বাস্থ্যকেন্দ্রের কোড : মাঠকর্মীর নাম: মাঠকর্মীর পদবী: HA/FWA/NGO কর্মী মাঠকর্মীর স্বাক্ষর : তারিখ: সেবা প্রদানকারীর স্বাক্ষর: তারিখ:

Form-16_A মাঠকর্মীর কপি

তারিখ:

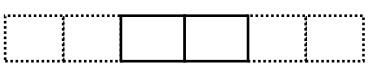

কুপন নং /রোগী সনাক্তকরণ নাম্বার:

\section{রেফারেল স্লিপ}

(মাঠকর্মী দ্বারা পূরণকৃত)

(বাম থেকে ডানে; জেলা কোড: ১ সংখ্যা, উপজেলা কোড: ২ সংখ্যা, ইউনিয়ন কোড: ২ সংখ্যা, মাঠকর্মীর কোড: ৩ সংখ্যা, সেবা গ্রহণকারীর কোড: 8 সংখ্যা) সেবা গ্রহণকারীর নাম: $\square$ গর্ভবতী মা $\square$ নবজাতক $\square$ অনূর্ধ্ব ৫ বছর বয়সী শিও: রেফারেল সেবার কোড: রেফারেল সেবার কোড: গর্ভকালীন ঝুকিপূর্ণ সমস্যা=১; প্রসব=২; ঝুঁকিপূর্ণ প্রসব=৩; প্রসব পরবর্তী ঝুঁকিপূর্ণ সমস্যা=8; নবজাতকের ঝুঁকিপূর্ণ সমস্যা=৫; অনূর্ধ্ব ৫ বছর বয়সী শিশ্তর ঝুঁকিপূর সমস্যা=৬। রেফারকৃত স্বাস্থ্যকেন্দ্র: রেফারকৃত স্বাস্থ্যকেন্দ্রের কোড :

মাঠকর্মীর নাম: মাঠকর্মীর পদবী: HA/FWA/NGO কর্মী মাঠকর্মীর স্বাক্ষর : তারিখ: 
কুপন বিতরণকারীর কোড নং:

জেলা: গাইবান্ধা/কুড়িগ্রাম; উপজেলা:

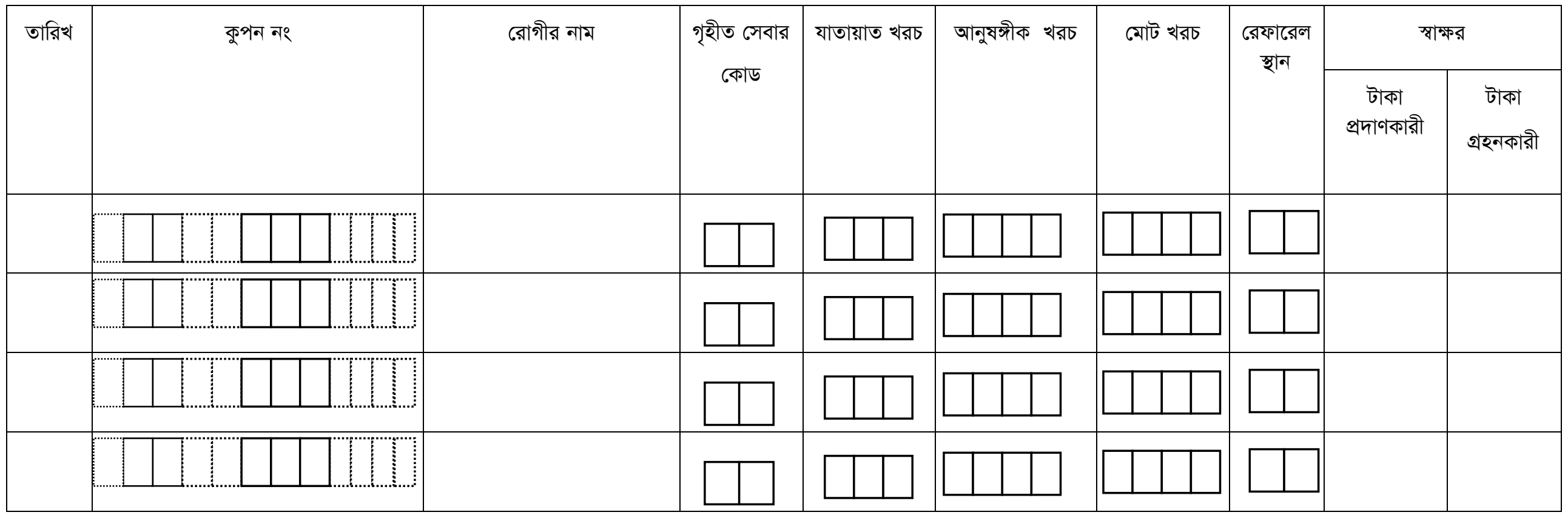

কুপন নং: (বাম থেকে ডানে): জেলা কোড: ১ সংখ্যা, উপজেলা কোড: ২ সংখ্যা, ইউনিয়ন কোড: ২ সংখ্যা, কুপন বিতরণকারীর কোড: ৩ সংখ্যা, কুপন এ্রহণকারীর কোড: 8 সংখ্যা

সেবার কোড: প্রসব পূর্ব ১ম সেবা=০১; প্রসব পূর্ব ২য় সেবা=০২; প্রসব পূর্ব ৩য় সেবা=০৩; প্রসবপূর্ব ৪র্থ সেবা=০৪; গর্ভকালীন জটিলতা ব্যবস্থাপনা =০৫; প্রসবকালীন সেবা=০৬; প্রসব পরবর্তী জটিলতা ব্যবস্থাপনা =০৭; প্রসব পরবর্তী জটিলতা ব্যবস্থাপনার ২য় সাক্ষাৎ =০৮; নবজাতকের জটিলতা ব্যবস্থাপনা =০৯; নবজাতকের জটিলতা ব্যবস্থাপনার ২য় সাক্ষাৎ=১০; অনূর্ব্ব ৫ বছর বয়সী শিঞ্ডর জটিলতা ব্যবস্থাপনা =১১; অনূর্ধর্ব ৫ বছর বয়সী শিশ্ডর জটিলতা ব্যবস্থাপনার ২য় সাক্ষাৎ =১২।

রেফারেল স্থান: ফুলছড়ি উপজেলা স্বাস্থ্য কমপ্লেক্স =০১; সাঘাটা উপজেলা স্বাস্থ্য কমপ্লেক্স =০২; সুন্দরগঞ্জ উপজেলা স্বাস্থ্য কমপ্লেক্স =০৩; গাইবান্ধা জেলা হাসপাতাল =০৪ ভুরুঙ্গামারী উপজেলা স্বাস্থ্য কমপ্লেক্স =০৫; চিলমারী উপজেলা স্বাস্থ্য কমপ্লেক্স =০৬; নাগেশ্বরী উপজেলা স্বাস্থ্য কমপ্লেক্স =০৭; কুড়িগ্রাম জেলা হাসপাতাল =০৮। 
কুপন বিতরণকারীর নাম:

কুপন বিতরণকারীর কোড নং

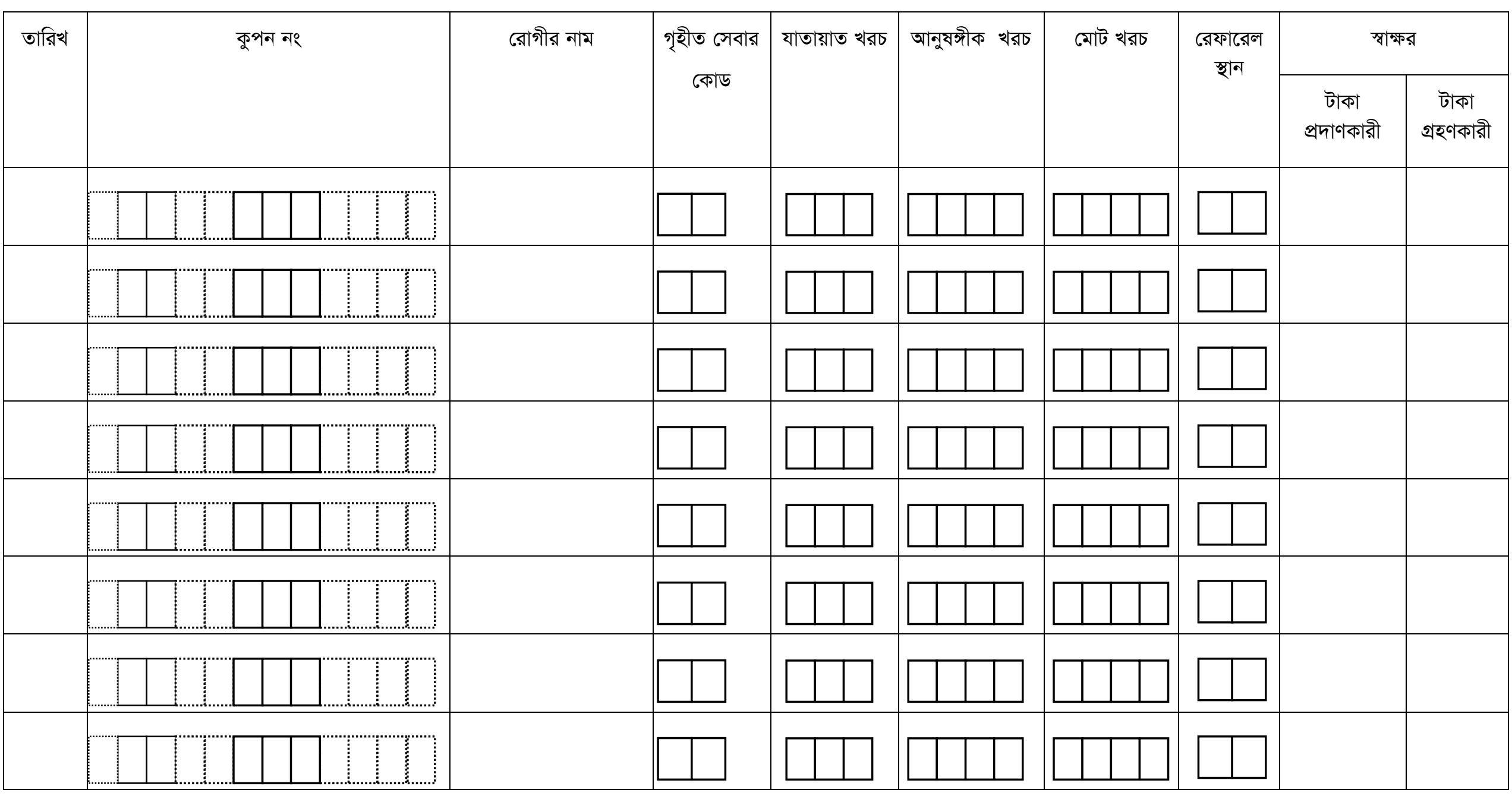

কুপন বিতরণকারীর পদবী: HA/FWA/NGO কর্মী জেলা: গাইবান্ধা/কুড়িগ্রাম; উপজেলা:
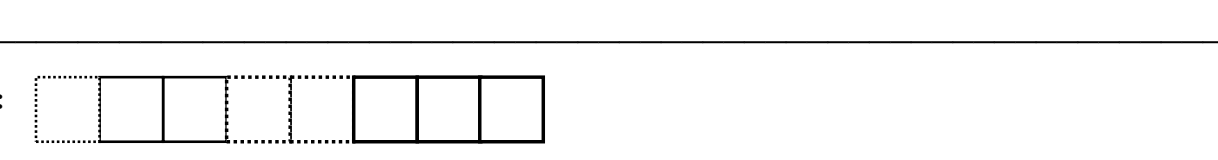
মাঠকর্মীর নাম:

মাঠকর্মীর পদবী: FWA/HA/NGO কর্মী

মাস: বছর:

মাঠকর্মীর কোড নং:

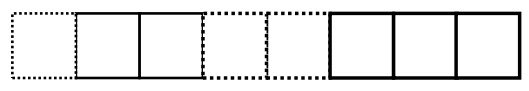

জেলা: গাইবান্ধা/কুড়িগ্রাম/জামালপুর; উপজেলা:

\begin{tabular}{|l|l|l|l|l|l|l|}
\hline তারিখ & \multicolumn{3}{|c|}{ রোগী সনাক্তকরণ নাম্বার } & রুাগীর নাম বিতরণ & রেফারেল স্থান গৃহীত লেবার কোড \\
\hline & & & \\
\hline
\end{tabular}

মোট বিতরণকৃত কুপন সংখ্যা ও টাকার পরিমান:

$\mathrm{x} 20=$

মোট সফল রেফারেল সংখ্যা ও টাকার পরিমান:

x $50=$

মোট টাকার পরিমান: কুপন + রেফারেল =

টাকা প্রদাণকারীর নামः

স্বাক্ষর:

টাকা গ্রহনকারীর নামः

স্বাক্ষর:

রোগী সনাক্তকরণ নাম্বার: (বাম থেকে ডানে: জেলা কোড: ১ সংখ্যা, উপজেলা কোড: ২ সংখ্যা, ইউনিয়ন কোড: ২ সংখ্যা, মাঠকর্মীর কোড: ৩ সংখ্যা, সেবা গ্রহণকারীর কোড: 8 সংখ্যা)

রেফারকারীর কোড নং (বাম থেকে ডানে): জেলা কোড: ১ সংখ্যা, উপজেলা কোড: ২ সংখ্যা, ইউনিয়ন কোড: ২ সংখ্যা, কুপন বিতরণকারী কোড: ৩ সংখ্যা ।

রেফারেল সেবার কোড: গর্ভকালীন ঝুকিপূর্ণ সমস্যা=১; প্রসব=২; ঝুঁকিপূর্ণ প্রসব=৩; প্রসব পরবর্তী ঝুঁকিপূর্ণ সমস্যা=8; নবজাতকের ঝুঁকিপূর্ণ সমস্যা=৫; অনূর্ধ ৫ বছর বয়সী শিশ্ডর ঝুঁকিপূর্ণ সমস্যা=৬।

রেফারেল স্থান: ফুলছড়ি উপজেলা স্বাস্থ্য কমপ্লেক্স =০১; সাঘাটা উপজেলা স্বাস্থ্য কমপ্লেক্স =০২; সুন্দরগঞ উপজেলা স্বাস্থ্য কমপ্লেক্স =০৩; গাইবান্ধা জেলা হাসপাতাল =০৪

ভুরুঞ্পামারী উপজেলা স্বাস্থ্য কমপ্লেক্স =০৫; চিলমারী উপজেলা স্বাস্থ্য কমপ্লেক্স =০৬; নাগেশ্বরী উপজেলা স্বাস্থ্য কমপ্লেক্স =০৭; কুড়িগ্রাম জেলা হাসপাতাল =০৮-

বকশীগঞ উপজেলা স্বাস্থ্য কমপ্লেক্স =০৯; ইসলামপুর উপজেলা স্বাস্থ্য কমপ্লেক্স =১০; মেলান্দহ উপজেলা স্বাস্থ্য কমপ্লেক্স =১১; জামালপুর জেলা হাসপাতাল =১২ 
মাঠকর্মীর নাম:

মাঠকর্মীর পদবী: HA/FWA/ NGO কর্মী

মাস:

মাঠকর্মীর কোড নং:

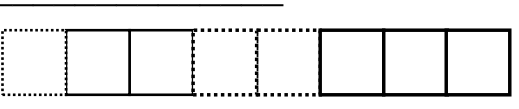

জেলা: গাইবান্ধা/কুড়িগ্গাম/জামালপুর; উপজেলা:

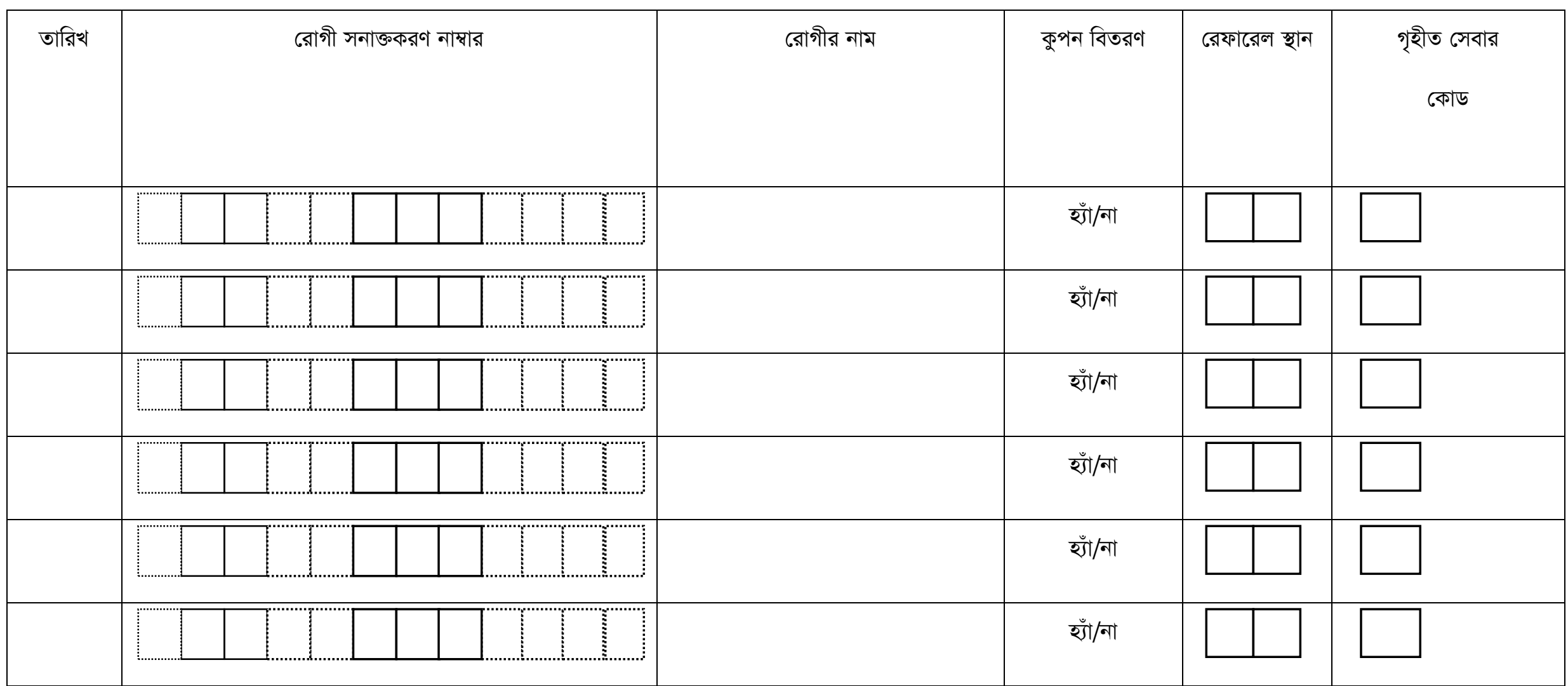

মোট বিতরণকৃত কুপন সংখ্যা ও টাকার পরিমান:

$x 20=$

মোট সফল রেফারেল সংখ্যা ও টাকার পরিমান:

x $50=$

মোট টাকার পরিমান: কুপন + রেফারেল =

টাকা প্রদাণকারীর নাম:

স্বাক্ষর:

টাকা গ্রহননকারীর নাম:

স্বাক্ষর: 
স্বাস্থ্যকেন্দ্র :

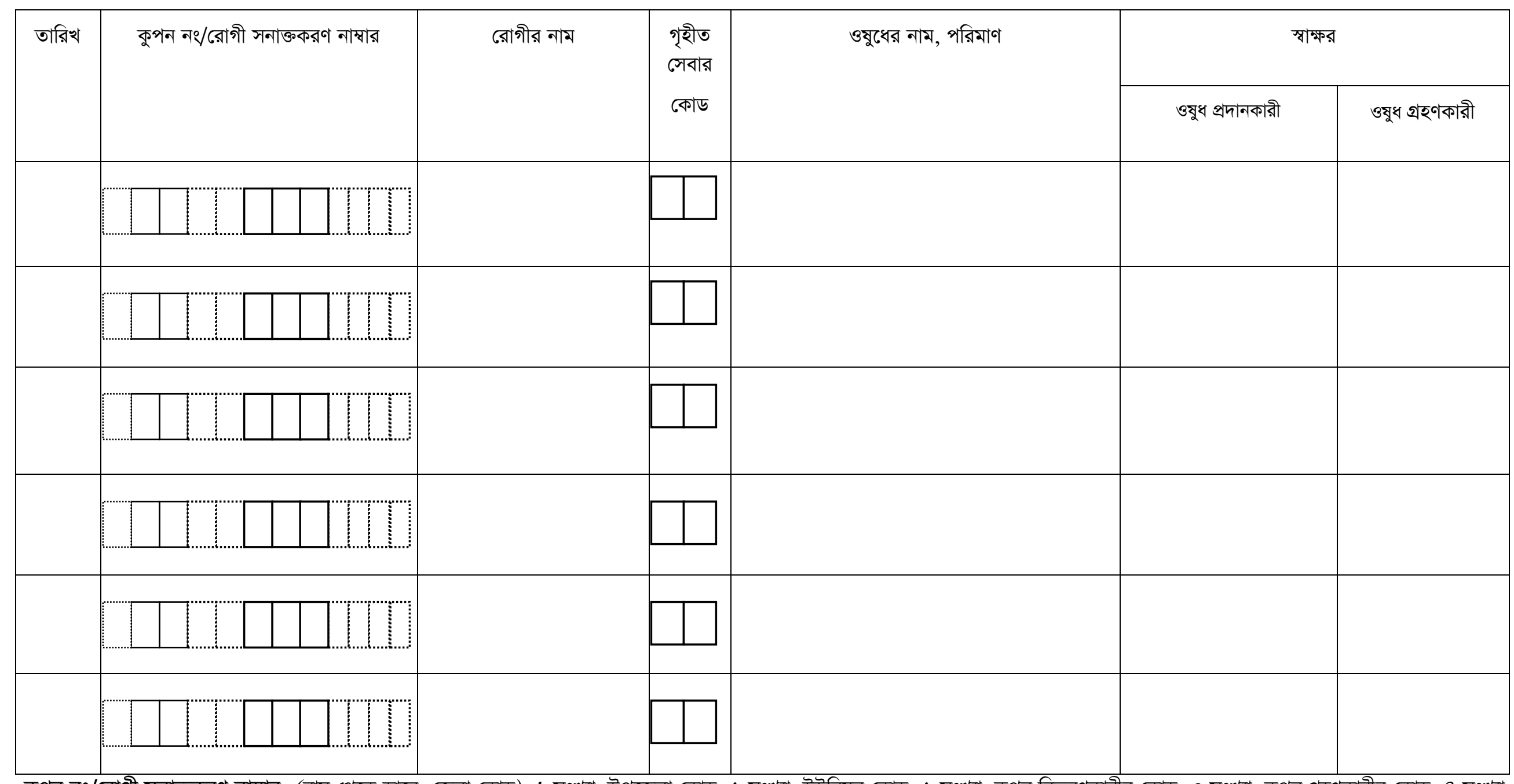

কুপন নংরোগী সনাক্তকরণ নাম্বার: (বাম থেকে ডানে: জেলা কোড): ১ সংখ্যা, উপজেলা কোড: ২ সংখ্যা, ইউনিয়ন কোড: ২ সংখ্যা, কুপন বিতরণকারীর কোড: ৩ সংখ্যা, কুপন গ্রহণকারীর কোড: ৪ সংখ্যা গৃহীত সেবার কোড: ০১ = প্রসব পূর্ব ১ম সেবা; ০২ = থ্রসব পূর্ব ২য় সেবা; ০৩ = প্রসব পূর্ব ৩য় সেবা; ০৪ = প্রসব পূর্ব ৪র্থ সেবা; ০৫ = গর্ভকালীন ঝুঁকিপূর্ণ সমস্যা; ০৬ = ঝুঁকিপূর্ণ প্রসব; ০৭ = প্রসব পরবর্তী ঝুঁকিপূর্ণ সমস্যা; ০৮- = প্রসব পরবর্তী ঝুঁকিপূর্ণ সমস্যার ২য় সাক্ষাৎ; ০৯ = নবজাতকের ঝুঁকিপূর্ণ সমস্যা; ১০ = নবজাতকের ঝুঁকিপূর্ণ সমস্যার ২য় স্বাক্ষাৎ; ১১ = অনূর্ধ্ব ৫-বছর বয়সী শিক্ডর ঝুঁকিপূর্ণ সমস্যা; ১২ = অনূর্ধ্ব ৫-বছর বয়সী শিশ্তর ঝুঁকিপূর্ণ সমস্যার ২য় স্বাক্ষাৎ। 
Form-16_D

স্বাস্থ্যকেন্দ্র :

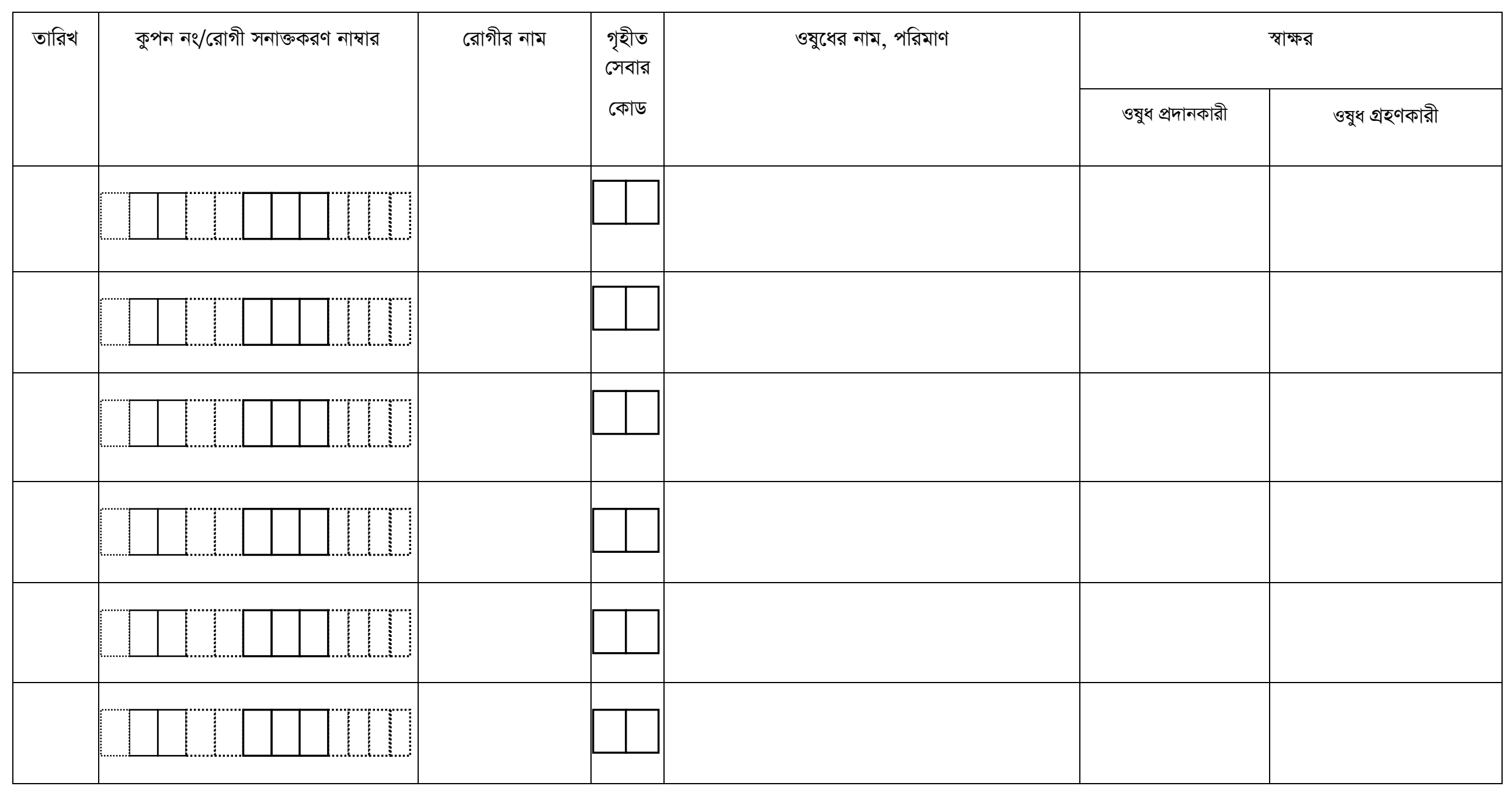

জেলা: গাইবান্ধা/কুড়িগ্রাম/জামালপুর; উপজেলা:

ওযুধ (DCM) প্রদানের রেজিষ্টার 
কুপন প্রদাণকারীর নাম:

জেলা: গাইবান্ধা/কুড়িগ্গাম; উপজেলা:

কুপন প্রদাণকারীর কোড নং:

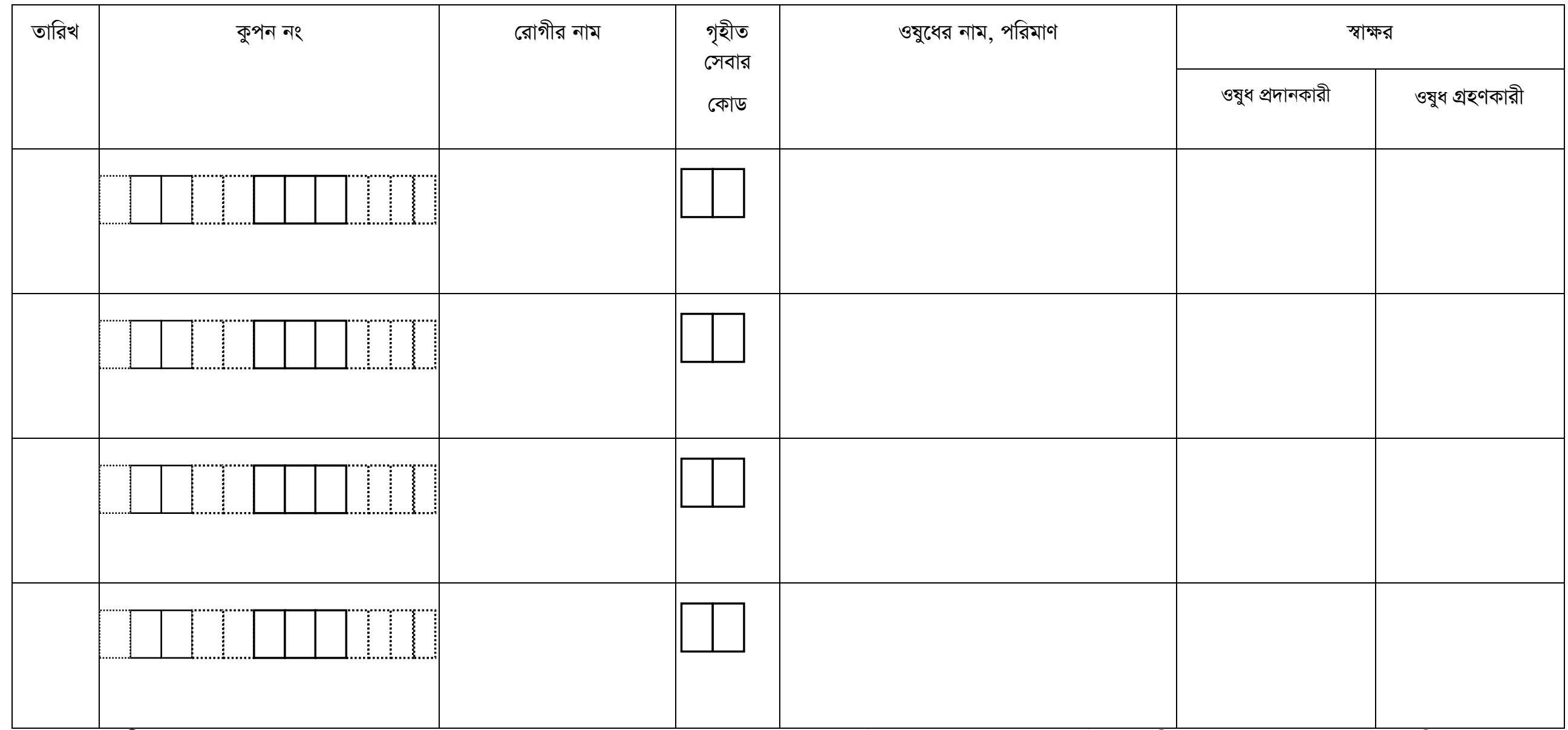

কুপন নং/রোগী সনাক্তকরণ নাম্বার: (বাম থেকে ডানে): জেলা কোড: ১ সংখ্যা, উপজেলা কোড: ২ সংখ্যা, ইউনিয়ন কোড: ২ সংখ্যা, কুপন বিতরণকারীর কোড: ৩ সংখ্যা, কুপন গ্রহণকারীর কোড: ৪ সংখ্যা গৃহীত সেবার কোড: ১ = প্রসব পূর্ব ১ম সেবা; ২ = প্রসব পূর্ব ২য় সেবা; ৩ = প্রসব পূর্ব ৩য় সেবা; 8 = প্রসব পূর্ব ৪র্থ সেবা; ৫ = গর্ভকালীন ঝুঁকিপূর্ণ সমস্যা; ৬ = ঝুঁকিপূর্ণ প্রসব;

$৭=$ প্রসব পরবর্তী ঝুঁকিপূর্ণ সমস্যা; b- = প্রসব পরবর্তী ঝুঁকিপূর্ণ সমস্যার ২য় সাক্ষাৎ; ৯ = নবজাতকের ঝুঁকিপূর্ণ সমস্যা; ১০ = নবজাতকের ঝুঁকিপূর্ণ সমস্যার ২য় স্বাক্ষাৎ;

১১ = অনূর্ধ্ব ৫-বছর বয়সী শিঞ্ডর ঝুঁকিপূর্ণ সমস্যা; ১২ = অনূর্ধ্ব ৫-বছর বয়সী শিশ্ডর ঝুঁকিপূর্ণ সমস্যার ২য় স্বাক্ষাৎ 
Form-16_E

কুপন প্রদাণকারীর নামः

কুপন প্রদাণকারীর কোড নং:

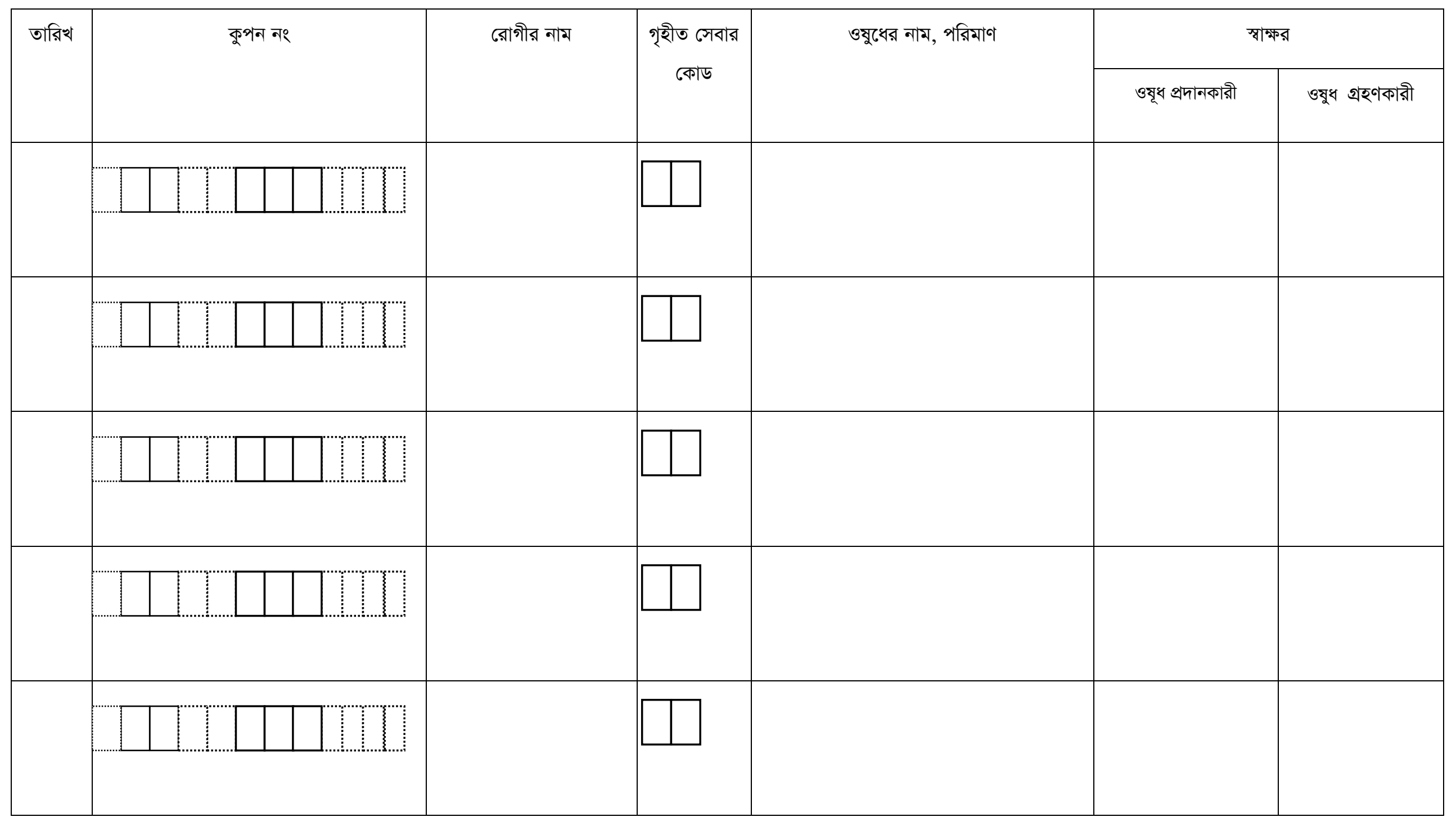

কুপন ব্যবহারকারীর জন্য ওষুধ প্রদানের রেজিষ্টার

জেলা: গাইবান্ধা/কুড়িগ্গাম; উপজেলা: 


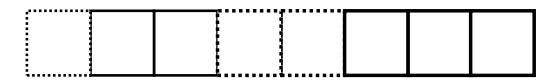

স্বাস্থ্যকেন্দ্র / ডায়াগনষ্টিক সেবাকেন্দ্র :

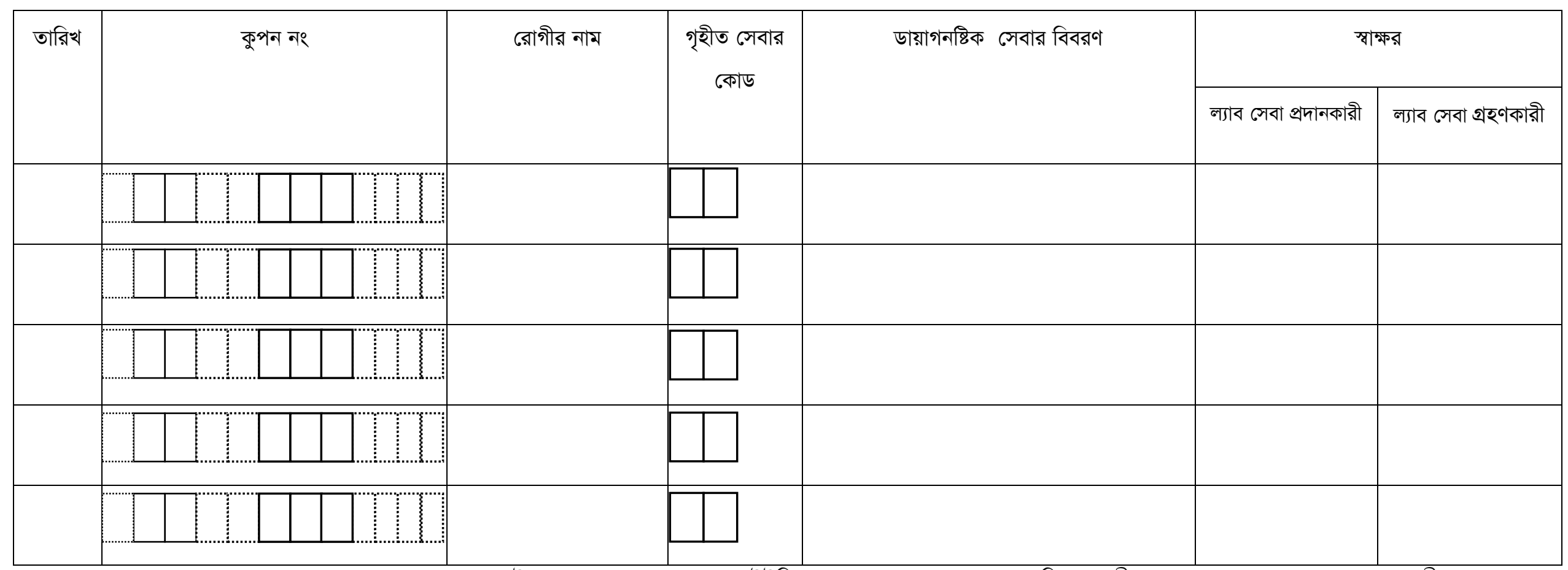

কুপন নং: (বাম থেকে ডানে): জেলা কোড: ১ সংখ্যা, উপজেলা কোড: ২ সংখ্যা, ইউনিয়ন কোড: ২ সংখ্যা, কুপন বিতরণকারীর কোড: ৩ সংখ্যা, কুপন গ্রহণকারীর কোড: 8 সংখ্যা সেবার কোড: প্রসব পূর্ব ১ম সেবা=০১; প্রসব পূর্ব ২য় সেবা=০২; প্রসব পূর্ব ৩য় সেবা=০৩; প্রসবপূর্ব ৪র্থ সেবা=০৪; গর্ভকালীন জটিলতা ব্যবস্থাপনা =০৫; প্রসবকালীন সেবা=০৬; প্রসব পরবর্তী জটিলতা ব্যবস্থাপনা =০৭; প্রসব পরবর্তী জটিলতা ব্যবস্থাপনার ২য় সাক্ষাৎ =০b; নবজাতকের জটিলতা ব্যবস্থাপনা =০৯; নবজাতকের জটিলতা ব্যবস্থাপনার ২য় সাক্ষাৎ=১০; অনূর্ধ্ব ৫ বছর বয়সী শিশ্ডর জটিলতা ব্যবস্থাপনা =১১; অনূর্ব্ব ৫ বছর বয়সী শিশুর জটিলতা ব্যবস্থাপনার ২য় সাক্ষাৎ =১২। 
উপজেলা:

কুপন প্রদাণকারীর কোড নং:

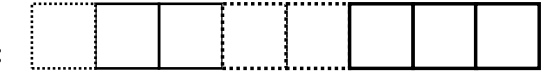

স্বাস্থ্যকেন্দ্র / ডায়াগনষ্টিক সেবাকেন্দ্র :

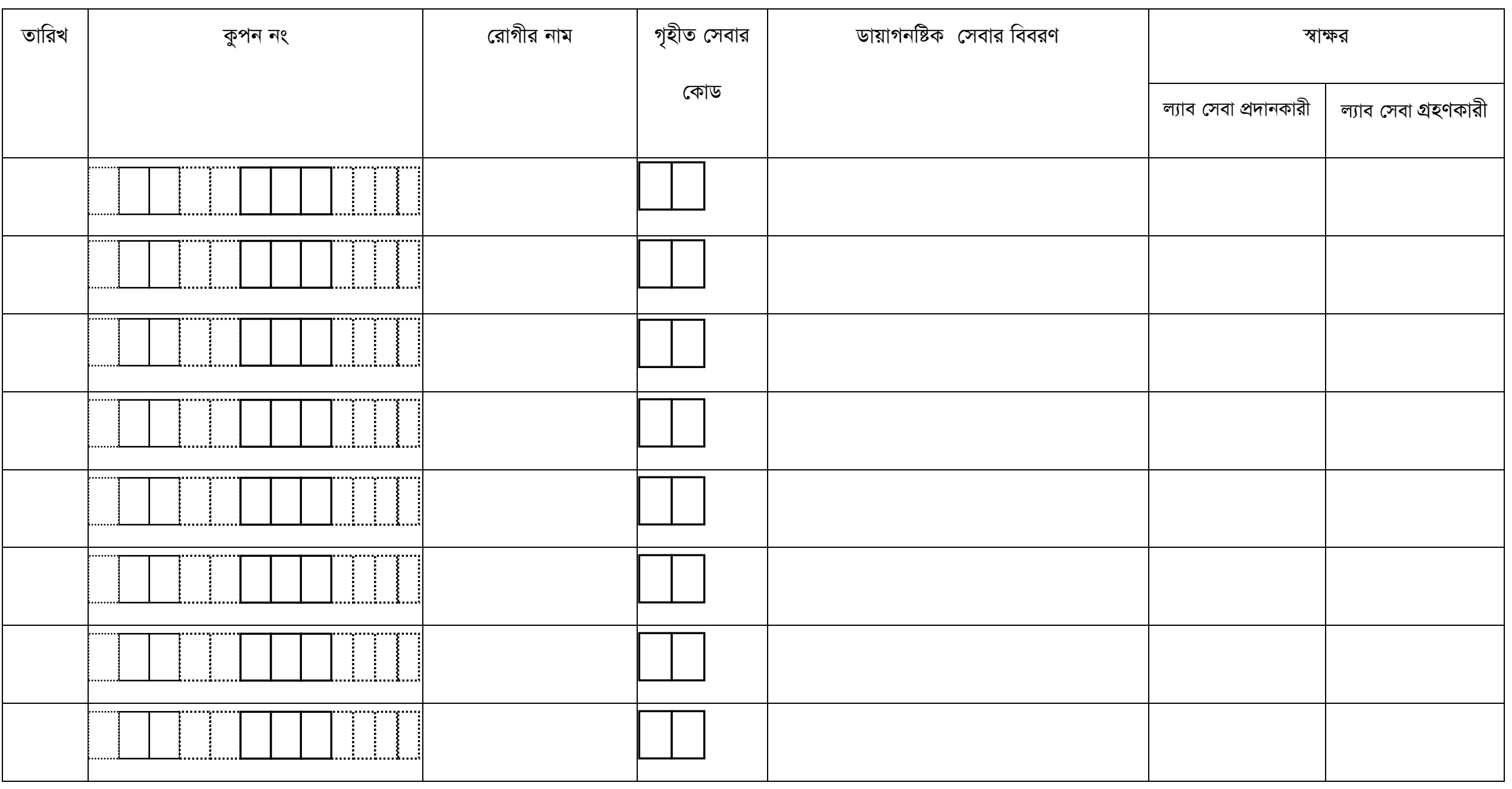


Form-

17_A

Introducing Pay-for-Performance (P4P) Approach to Increase Utilization of Maternal, Newborn and Child Health Services in Bangladesh

Name of the Facility/ Cost Centre:

\section{Petty Cash Register}

Month :

Year:

\begin{tabular}{|c|c|c|c|c|}
\hline Date & Voucher No. and Particulars & Debit & Credit & Total \\
\hline & & & & \\
\hline & & & & \\
\hline & & & & \\
\hline & & & & \\
\hline & & & & \\
\hline & & & & \\
\hline & & & & \\
\hline & & & & \\
\hline & & & & \\
\hline & & & & \\
\hline & & & & \\
\hline & & & & \\
\hline & & & & \\
\hline & & & & \\
\hline & & & & \\
\hline & & & & \\
\hline & & & & \\
\hline & & & & \\
\hline & & & & \\
\hline & & & & \\
\hline
\end{tabular}


Form-17_B

Introducing Pay-for-Performance (P4P) Approach to Increase Utilization of Maternal, Newborn and Child Health Services in Bangladesh

\section{Name of the Facility/ Cost Centre:}

\section{Payment Voucher}

Month : Year:

\begin{tabular}{|c|c|c|c|}
\hline Date: & Description & $\begin{array}{c}\text { HoE Ref. } \\
\#\end{array}$ & Amount in Taka \\
\hline \multicolumn{4}{|l|}{ Check No.: } \\
\hline \multicolumn{4}{|l|}{$\begin{array}{l}\text { Voucher } \\
\text { No.:PCV/CV: }\end{array}$} \\
\hline In words : & Total Amount in Taka : & & \\
\hline \multicolumn{4}{|l|}{ Payee : } \\
\hline \multicolumn{4}{|c|}{ Purpose/Description : } \\
\hline \multicolumn{4}{|l|}{ Approvals } \\
\hline Prepared by : & Signature & & Date: \\
\hline & & & \\
\hline & & & \\
\hline Approved by: & Signature & & Date: \\
\hline
\end{tabular}

HoE Ref. \# - Head of Expenditure Reference number. Its description is given below. Ref numbers 4-7 are not applicable for Jamalpur.

HoE Ref. Code:1-Incentive for MNCH Team; 2- Incentive for fieldworkers; 3-Drugs Consumables and Maintenance (DCM) and other fund; 4-Transportation costs for coupon beneficiaries; 5-Medicine costs for coupon beneficiaries; Incidental expenses for coupon beneficiaries; 7-Diagonestic costs for coupon beneficiaries 
Introducing Pay-for-Performance (P4P) Approach to Increase Utilization of

Maternal, Newborn and Child Health Services in Bangladesh

\section{Name of the Facility/Cost Centre:}

\section{Bank Book}

Month :

Year:

\begin{tabular}{|c|c|c|c|c|c|c|c|c|c|c|}
\hline \multicolumn{5}{|c|}{ Debit } & \multicolumn{5}{|c|}{ Credit } & \multirow{2}{*}{ Balance } \\
\hline Date & $\begin{array}{l}\text { Voucher } \\
\text { No. }\end{array}$ & $\begin{array}{l}\text { Particulars with } \\
\text { check number }\end{array}$ & $\begin{array}{l}\text { Ledger } \\
\text { Folio }\end{array}$ & $\begin{array}{c}\text { Debit } \\
\text { amount }\end{array}$ & Date & $\begin{array}{c}\text { Voucher } \\
\text { No. }\end{array}$ & $\begin{array}{l}\text { Particulars with } \\
\text { check number }\end{array}$ & $\begin{array}{c}\text { Ledger } \\
\text { Folio }\end{array}$ & $\begin{array}{c}\text { Credit } \\
\text { amount }\end{array}$ & \\
\hline & & & & Tk. & & & & & Tk. & Tk. \\
\hline & & Opening balance & & & & & & & & \\
\hline & & $\begin{array}{l}\text { Fund transfer } \\
\text { from PC }\end{array}$ & & & & & & & & \\
\hline & & & & & & & & & & \\
\hline & & & & & & & & & & \\
\hline & & & & & & & & & & \\
\hline & & & & & & & & & & \\
\hline & & & & & & & & & & \\
\hline & & & & & & & & & & \\
\hline & & & & & & & Closing balance & & & \\
\hline & & & & & & & & & & \\
\hline
\end{tabular}


Introducing Pay-for-Performance (P4P) Approach to Increase Utilization of Maternal, Newborn and Child Health Services in Bangladesh

Name of the Facility/ Cost Centre:

Cash

Book

Month :

Year:

\begin{tabular}{|c|c|c|c|c|c|c|c|c|c|c|}
\hline \multicolumn{5}{|c|}{ Debit } & \multicolumn{5}{|c|}{ Credit } & \multirow{2}{*}{ Balance } \\
\hline Date & $\begin{array}{l}\text { Voucher } \\
\text { No. }\end{array}$ & $\begin{array}{l}\text { Particulars with } \\
\text { check number }\end{array}$ & $\begin{array}{l}\text { Ledger } \\
\text { Folio }\end{array}$ & $\begin{array}{c}\text { Debit } \\
\text { amount }\end{array}$ & Date & $\begin{array}{c}\text { Voucher } \\
\text { No. }\end{array}$ & $\begin{array}{l}\text { Particulars with } \\
\text { check number }\end{array}$ & $\begin{array}{l}\text { Ledger } \\
\text { Folio }\end{array}$ & $\begin{array}{l}\text { Credit } \\
\text { amount }\end{array}$ & \\
\hline & & & & Tk. & & & & & Tk. & Tk. \\
\hline & & $\begin{array}{l}\text { Petty Cash fund } \\
\text { for Several } \\
\text { expenses,CHQ- } \\
5898960\end{array}$ & & & & & & & & \\
\hline & & & & & & & $\begin{array}{l}\text { DCM \& Other } \\
\text { fund }\end{array}$ & & & \\
\hline & & & & & & & $\begin{array}{l}\text { Incentive for } \\
\text { Fieldworkers }\end{array}$ & & & \\
\hline & & & & & & & & & & \\
\hline & & & & & & & Closing balance & & & \\
\hline & & & & & & & & & & \\
\hline & & & & & & & & & & \\
\hline
\end{tabular}


Form-17_E

Introducing Pay-for-Performance (P4P) Approach to Increase Utilization of Maternal, Newborn and Child Health Services in Bangladesh

Name of the Facility/ Cost Centre:

Ledger Book: DCM and Other Fund

Month :

Year:

\begin{tabular}{|c|c|c|c|c|}
\hline Date & Particulars & $\begin{array}{l}\text { Cash/bank } \\
\text { book Folio }\end{array}$ & $\begin{array}{l}\text { Credit } \\
\text { Amount (Tk) }\end{array}$ & $\begin{array}{l}\text { Total } \\
\text { Amount } \\
\text { (Tk) }\end{array}$ \\
\hline & & & & \\
\hline & & & & \\
\hline & & & & \\
\hline & & & & \\
\hline & & & & \\
\hline & & & & \\
\hline & & & & \\
\hline & & & & \\
\hline & & & & \\
\hline & & & & \\
\hline & & & & \\
\hline & & & & \\
\hline & & & & \\
\hline & & & & \\
\hline & & & & \\
\hline & & & & \\
\hline & & & & \\
\hline & & & & \\
\hline & & & & \\
\hline & & & & \\
\hline & & & & \\
\hline & & & & \\
\hline
\end{tabular}


Form-17_F

Introducing Pay-for-Performance (P4P) Approach to Increase Utilization of

Maternal, Newborn and Child Health Services in Bangladesh

Name of the Facility/ Cost Centre:

Ledger Book: Incentive Payment to Field Worker

Month :

Year:

\begin{tabular}{|c|c|c|c|c|}
\hline Date & Particulars & $\begin{array}{l}\text { Cash/bank } \\
\text { book Folio }\end{array}$ & $\begin{array}{c}\text { Credit } \\
\text { Amount } \\
(\mathrm{Tk})\end{array}$ & $\begin{array}{c}\text { Total } \\
\text { Amount } \\
(\mathrm{Tk})\end{array}$ \\
\hline & & & & \\
\hline & & & & \\
\hline & & & & \\
\hline & & & & \\
\hline & & & & \\
\hline & & & & \\
\hline & & & & \\
\hline & & & & \\
\hline & & & & \\
\hline & & & & \\
\hline & & & & \\
\hline & & & & \\
\hline & & & & \\
\hline & & & & \\
\hline & & & & \\
\hline & & & & \\
\hline & & & & \\
\hline & & & & \\
\hline & & & & \\
\hline & & & & \\
\hline & & & & \\
\hline
\end{tabular}


Form-17_G

Introducing Pay-for-Performance (P4P) Approach to Increase Utilization of Maternal, Newborn and Child Health Services in Bangladesh

Name of the Facility/ Cost Centre:

Ledger Book: Incentive Payment to MNCH Team

Month :

Year:

\begin{tabular}{|c|c|c|c|c|}
\hline Date & Particulars & $\begin{array}{l}\text { Cash/bank } \\
\text { book Folio }\end{array}$ & $\begin{array}{l}\text { Credit } \\
\text { Amount } \\
(\mathrm{Tk}) \\
\end{array}$ & $\begin{array}{l}\text { Total } \\
\text { Amount } \\
\text { (Tk) }\end{array}$ \\
\hline & & & & \\
\hline & & & & \\
\hline & & & & \\
\hline & & & & \\
\hline & & & & \\
\hline & & & & \\
\hline & & & & \\
\hline & & & & \\
\hline & & & & \\
\hline & & & & \\
\hline & & & & \\
\hline & & & & \\
\hline & & & & \\
\hline & & & & \\
\hline & & & & \\
\hline & & & & \\
\hline & & & & \\
\hline & & & & \\
\hline & & & & \\
\hline
\end{tabular}


Form-17_H

Introducing Pay-for-Performance (P4P) Approach to Increase Utilization of Maternal, Newborn and Child Health Services in Bangladesh

Name of the Facility/ Cost Centre:

Stock Register for DCM

Month :

Year:

Name of Item:

\begin{tabular}{|c|c|c|c|c|c|c|c|c|}
\hline Date & B.F. & Received & $\begin{array}{l}\text { C. Memo } \\
\text { Challan No. }\end{array}$ & Total & $\begin{array}{l}\text { Requisition } \\
\text { No. }\end{array}$ & $\begin{array}{l}\text { Quantity } \\
\text { Disbursed }\end{array}$ & Balance & $\begin{array}{l}\text { Remar } \\
\text { ks }\end{array}$ \\
\hline & & & & & & & & \\
\hline & & & & & & & & \\
\hline & & & & & & & & \\
\hline & & & & & & & & \\
\hline & & & & & & & & \\
\hline & & & & & & & & \\
\hline & & & & & & & & \\
\hline & & & & & & & & \\
\hline & & & & & & & & \\
\hline & & & & & & & & \\
\hline & & & & & & & & \\
\hline & & & & & & & & \\
\hline & & & & & & & & \\
\hline & & & & & & & & \\
\hline & & & & & & & & \\
\hline & & & & & & & & \\
\hline & & & & & & & & \\
\hline & & & & & & & & \\
\hline
\end{tabular}


Form-17_I

Introducing Pay-for-Performance (P4P) Approach to Increase Utilization of

Maternal, Newborn and Child Health Services in Bangladesh

Name of the Facility/ Cost Centre:

Stock Register of Medicines Coupon for Beneficiaries

Month :

Year:

Name of

Medicine:

\begin{tabular}{|c|c|c|c|c|c|c|c|c|}
\hline Date & B.F. & Received & $\begin{array}{l}\text { C. Memo } \\
\text { Challan No. }\end{array}$ & Total & $\begin{array}{c}\text { Requisition } \\
\text { No. }\end{array}$ & $\begin{array}{c}\text { Quantity } \\
\text { Disburse } \\
\text { d }\end{array}$ & Balance & Remarks \\
\hline & & & & & & & & \\
\hline & & & & & & & & \\
\hline & & & & & & & & \\
\hline & & & & & & & & \\
\hline & & & & & & & & \\
\hline & & & & & & & & \\
\hline & & & & & & & & \\
\hline & & & & & & & & \\
\hline & & & & & & & & \\
\hline & & & & & & & & \\
\hline & & & & & & & & \\
\hline & & & & & & & & \\
\hline & & & & & & & & \\
\hline & & & & & & & & \\
\hline & & & & & & & & \\
\hline & & & & & & & & \\
\hline & & & & & & & & \\
\hline & & & & & & & & \\
\hline
\end{tabular}


Form-17_J

Introducing Pay-for-Performance (P4P) Approach to Increase Utilization of

Maternal, Newborn and Child Health Services in Bangladesh

Name of the Facility/ Cost Centre:

Ledger Book: Transportation for Coupon Beneficiaries

Month :

Year:

\begin{tabular}{|c|c|c|c|c|}
\hline Date & Particulars & $\begin{array}{l}\text { Cash/bank } \\
\text { book Folio }\end{array}$ & $\begin{array}{c}\text { Credit } \\
\text { Amount } \\
\text { (Tk) }\end{array}$ & $\begin{array}{c}\text { Total } \\
\text { Amount } \\
\text { (Tk) }\end{array}$ \\
\hline & & & & \\
\hline & & & & \\
\hline & & & & \\
\hline & & & & \\
\hline & & & & \\
\hline & & & & \\
\hline & & & & \\
\hline & & & & \\
\hline & & & & \\
\hline & & & & \\
\hline & & & & \\
\hline & & & & \\
\hline & & & & \\
\hline & & & & \\
\hline & & & & \\
\hline & & & & \\
\hline & & & & \\
\hline & & & & \\
\hline & & & & \\
\hline & & & & \\
\hline & & & & \\
\hline
\end{tabular}


Form-17_K

Introducing Pay-for-Performance (P4P) Approach to Increase Utilization of

Maternal, Newborn and Child Health Services in Bangladesh

Name of the Facility/ Cost Centre:

Ledger Book: Medicine for Coupon Beneficiaries

Month :

Year:

\begin{tabular}{|c|c|c|c|c|}
\hline Date & Particulars & $\begin{array}{l}\text { Cash/bank } \\
\text { book Folio }\end{array}$ & $\begin{array}{c}\text { Credit } \\
\text { Amount } \\
(\mathrm{Tk})\end{array}$ & $\begin{array}{c}\text { Total } \\
\text { Amount } \\
(\mathrm{Tk})\end{array}$ \\
\hline & & & & \\
\hline & & & & \\
\hline & & & & \\
\hline & & & & \\
\hline & & & & \\
\hline & & & & \\
\hline & & & & \\
\hline & & & & \\
\hline & & & & \\
\hline & & & & \\
\hline & & & & \\
\hline & & & & \\
\hline & & & & \\
\hline & & & & \\
\hline & & & & \\
\hline & & & & \\
\hline & & & & \\
\hline & & & & \\
\hline & & & & \\
\hline & & & & \\
\hline
\end{tabular}


Form-17_L

Introducing Pay-for-Performance (P4P) Approach to Increase Utilization of

Maternal, Newborn and Child Health Services in Bangladesh

Name of the Facility/ Cost Centre:

Ledger Book: Incidental Costs for Coupon Beneficiaries

Month :

Year:

\begin{tabular}{|c|c|c|c|c|}
\hline Date & Particulars & $\begin{array}{l}\text { Cash/bank } \\
\text { book Folio }\end{array}$ & $\begin{array}{l}\text { Credit } \\
\text { Amount } \\
\text { (Tk) }\end{array}$ & $\begin{array}{l}\text { Total } \\
\text { Amount } \\
\text { (Tk) }\end{array}$ \\
\hline & & & & \\
\hline & & & & \\
\hline & & & & \\
\hline & & & & \\
\hline & & & & \\
\hline & & & & \\
\hline & & & & \\
\hline & & & & \\
\hline & & & & \\
\hline & & & & \\
\hline & & & & \\
\hline & & & & \\
\hline & & & & \\
\hline & & & & \\
\hline & & & & \\
\hline & & & & \\
\hline & & & & \\
\hline & & & & \\
\hline & & & & \\
\hline
\end{tabular}


Form-17_M

Introducing Pay-for-Performance (P4P) Approach to Increase Utilization of Maternal, Newborn and Child Health Services in Bangladesh

Name of the Facility/ Cost Centre:

Ledger Book: Diagnostic Costs at the Diagnostic Center for Coupon Beneficiaries Month : Year:

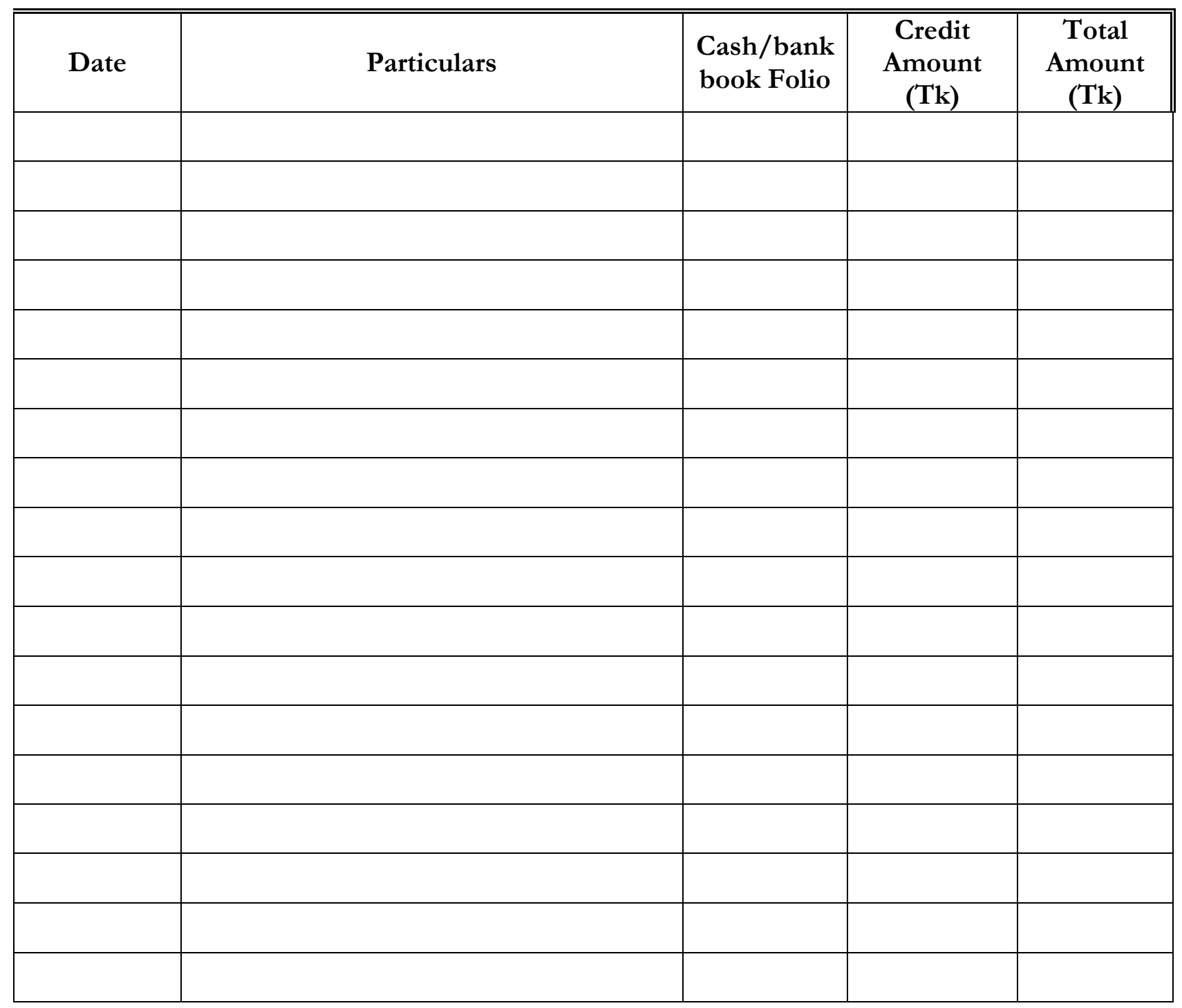


Form-17_N

Introducing Pay-for-Performance (P4P) Approach to Increase Utilization of Maternal, Newborn and Child Health Services in Bangladesh

Name of the Facility/ Cost Centre:

Ledger Book: Diagnostic Costs at the Facility for Coupon Beneficiaries

Month :

Year:

\begin{tabular}{|c|c|c|c|c|}
\hline Date & Particulars & $\begin{array}{l}\text { Cash/bank } \\
\text { book Folio }\end{array}$ & $\begin{array}{c}\text { Credit } \\
\text { Amount } \\
\text { (Tk) }\end{array}$ & $\begin{array}{c}\text { Total } \\
\text { Amount } \\
\text { (Tk) }\end{array}$ \\
\hline & & & & \\
\hline & & & & \\
\hline & & & & \\
\hline & & & & \\
\hline & & & & \\
\hline & & & & \\
\hline & & & & \\
\hline & & & & \\
\hline & & & & \\
\hline & & & & \\
\hline & & & & \\
\hline & & & & \\
\hline & & & & \\
\hline & & & & \\
\hline & & & & \\
\hline & & & & \\
\hline & & & & \\
\hline & & & & \\
\hline
\end{tabular}




\section{Form- 18}

Introducing Pay-for-Performance (P4P) Approach to Increase Utilization of Maternal, Newborn and Child Health Services in Bangladesh Name of the Facility/Cost Center:

\section{Comparison Statement*}

\begin{tabular}{|l|l|l|l|}
\hline \multirow{2}{*}{ Description of product/service } & \multicolumn{3}{|c|}{ Vendor Name and Address } \\
\hline \multirow{4}{*}{} & Vendor-1: & Vendor-2: & Vendor-3: \\
\cline { 2 - 5 } & Price details & Price details & Price details \\
\cline { 2 - 5 } & & & \\
\hline
\end{tabular}

Based on the comparison of the above three quotations for purchasing the item(s)

Vendor $(1 / 2 / 3)$ has been nominated and thus recommended to be ordered for

supplying the item(s) as of quotation mentioned price.

Certified By: (please tick) CS/UHFPO

Prepared By: (please tick) Head Assistant-cum-Accountant/Statistician/Cashier

Name and Signature:

Name and Signature:

Date:

Date:

*Enclosed: Original quotations printed in vendor's official pad having signature with seal. 
Name of Facility/Cost Center:

Date:

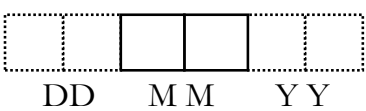

Sl. No.

DD M M Y Y

Coupon/Patient No: \begin{tabular}{|l|l|l|l|l|}
\hline & & & & \\
\hline
\end{tabular}

(From left to right, District code: 1 digit; Upazila code: 2 digits; Union code: 2 digits; Coupon Distributor/Fieldworker's code: 3 digits; Coupon Receiver/Patient's code: 4 digits)

Name: $\square$ Pregnant Women $\square$ Neonate $\square$ Under-5 child

Type of Medicine Fund: $\square \mathrm{MNCH} \quad \square \mathrm{DCM}$

Name and amount of Medicines:

Name of Service Provider:

Signature

Date:

Name of Pharmacist/Medicine Provider:

Signature

Date:

Signature/fingerprint of Patient/Coupon Recipient:

Date:

Approved by: (UHFPO/RMO)

Signature:

Date:

Form_20 Population Council Copy

Medicine Receipt

(To be prepared by Pharmacist/Medicine Provider)

Name of Facility/Cost Center:

Date:

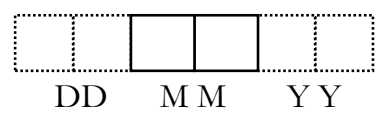

Coupon/Patient No: $\square$ (

(From left to right, District code: 1 digit; Upazila code: 2 digits; Union code: 2 digits; Coupon Distributor/Fieldworker's code: 3 digits; Coupon Receiver/Patient's code: 4 digits)

Name: $\square$ Pregnant Women $\square$ Neonate $\square$ Under-5 child

Type of Medicine Fund: $\square \mathrm{MNCH} \quad \square \mathrm{DCM}$

Name and amount of Medicines:

Name of Service Provider:

Signature

Date:

Name of Pharmacist/Medicine Provider:

Signature

Date:

Signature/fingerprint of Patient/Coupon Recipient:

Date:

Approved by: (UHFPO/RMO)

Signature: 
Form_20 Patient Copy

\section{Medicine Receipt}

(To be prepared by Pharmacist/Medicine Provider)

Name of Facility/Cost Center:

Date: $:$\begin{tabular}{|l|l|l}
$\square$ & \\
\hline $\mathrm{DM}$
\end{tabular}

DD M M YY

S1. No.

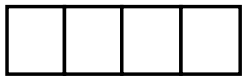

Coupon/Patient No:

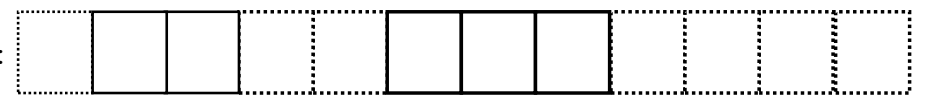

(From left to right, District code: 1 digit; Upazila code: 2 digits; Union code: 2 digits; Coupon Distributor/Fieldworker's code: 3 digits; Coupon Receiver/Patient's code: 4 digits)

Name: $\square$ Pregnant Women $\square$ Neonate $\quad \square$ Under- 5 child

Type of Medicine Fund: $\square \mathrm{MNCH} \quad \square \mathrm{DCM}$

Name and amount of Medicines:

Name of Service Provider:

Signature

Date:

Name of Pharmacist/Medicine Provider:

Signature

Date:

Signature/fingerprint of Patient/Coupon Recipient:

Date:

Approved by: (UHFPO/RMO)

Signature:

Date: 
Name of Facility/DTC:

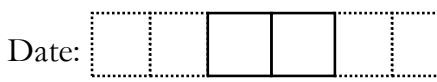

DD M M Y Y

Coupon/Patient No:

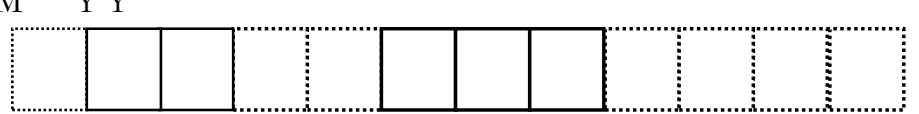

(From left to right, District code: 1 digit; Upazila code: 2 digits; Union code: 2 digits; Coupon Distributor/Fieldworker's code: 3 digits; Coupon Receiver/Patient's code: 4 digits)

Name: $\square$ Pregnant Women $\square$ Neonate $\square$ Under- 5 child

Name of Diagnostic Testing Center (DTC): $\square$ Facility $\square$ DTC (please specify)

Name of and reasons for test:

Name of Lab Technician: Signature

Date:

Date:

Approved by: (UHFPO/RMO)

Signature:

Date:

Form_21 Population Council Copy

\section{Diagnostic Service Receipt}

(To be prepared by Lab Technician)

Name of Facility/DTC:

Date:

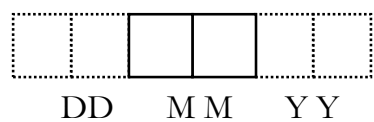

Sl. No.

DD M M Y Y

Coupon/Patient No: $\square=\square \quad l$

(From left to right, District code: 1 digit; Uparila code: 2 digits; Union code: 2 digits; Coupon Distributor/Fieldworker's code: 3 digits; Coupon Receiver/Patient's code: 4 digits)

Name: $\square$ Pregnant Women $\square$ Neonate $\square$ Under-5 child

Name of Diagnostic Testing Center (DTC): $\square$ Facility $\square$ DTC (please specify)

Name of and reasons for test:

Name of Lab Technician: Signature

Date:

Date: 
Form_21 Patient Copy

\section{Diagnostic Service Receipt}

(To be prepared by Lab Technician)

Name of Facility/DTC:

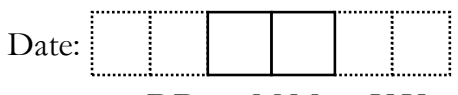

DD $\quad \mathrm{MM} \quad \mathrm{YY}$

Coupon/Patient No:

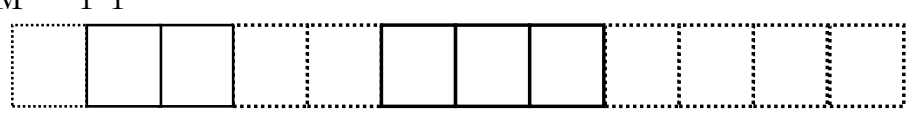

(From left to right, District code: 1 digit; Upazila code: 2 digits; Union code: 2 digits; Coupon Distributor/Fieldworker's code: 3 digits; Coupon Receiver/Patient's code: 4 digits)

Name: $\square$ Pregnant Women $\square$ Neonate $\quad \square$ Under-5 child

Name of Diagnostic Testing Center (DTC): $\square$ Facility $\square$ DTC (please specify)

Name of and reasons for test:

Name of Lab Technician:

Signature

Date:

Date:

Approved by: (UHFPO/RMO)

Signature:

Date:

Form_21 Diagnostic Center Copy

Diagnostic Service Receipt

(To be prepared by Lab Technician)

Name of Facility/DTC:

Date:

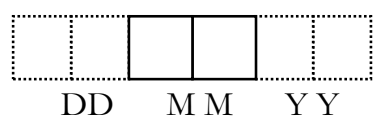

Sl. No.

DD M M YY

Coupon/Patient No:

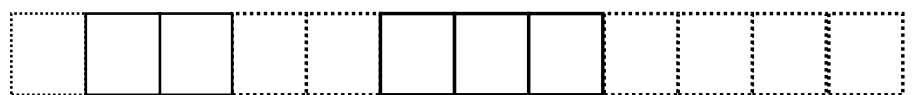

(From left to right, District code: 1 digit; Uparila code: 2 digits; Union code: 2 digits; Coupon Distributor/Fieldworker's code: 3 digits; Coupon Receiver/Patient's code: 4 digits)

Name: $\square$ Pregnant Women $\square$ Neonate $\square$ Under- 5 child

Name of Diagnostic Testing Center (DTC): $\square$ Facility $\square$ DTC (please specify)

Name of and reasons for test:

Name of Lab Technician:

Signature

Date:

Signature/fingerprint of Patient/Coupon Recipient:

Date:

Approved by: (UHFPO/RMO)

Signature:

Date: 
Introducing Pay-for-Performance (P4P) Approach to Increase Utilization of

Maternal, Newborn and Child Health Services in Bangladesh

\section{Name of the Facility/ Cost Centre:}

Expenditure Details for the Month: Year:

\begin{tabular}{|l|l|l|l|l|l|}
\hline $\begin{array}{c}\text { Voucher } \\
\text { Number }\end{array}$ & Check \# & Date & & Description & HoE Ref. \# \\
\hline & & & & & \\
\hline & & & & & \\
\hline & & & & & \\
\hline & & & & & \\
\hline & & & & & \\
\hline
\end{tabular}

HoE Ref. \# - Head of Expenditure Reference number. Its description is given below. Ref. numbers 4-7 are not applicable for Jamalpur.

HoE Ref. Code:1-Incentive for MNCH Team; 2- Incentive for fieldworkers; 3-Drugs Consumables and Maintenance (DCM) and other fund; 4-Transportation costs for coupon beneficiaries; 5-Medicine costs for coupon beneficiaries;

6- Incidental expenses for coupon beneficiaries; 7-Diagonestic costs for coupon beneficiaries 
Form-22_B

Introducing Pay-for-Performance (P4P) Approach to Increase Utilization of Maternal, Newborn and Child Health Services in Bangladesh

\section{Name of the Facility/Cost Centre:}

\section{Checks Outstanding Schedule}

As of last day of the Month Year

\begin{tabular}{|c|c|c|c|}
\hline $\begin{array}{c}\text { Check } \\
\text { Date }\end{array}$ & $\begin{array}{c}\text { Check } \\
\text { Number }\end{array}$ & Payee & $\begin{array}{c}\text { Amount in } \\
\text { Taka }\end{array}$ \\
\hline & & & \\
\hline & & & \\
\hline & & & \\
& & Total Amount in Taka & \\
\hline
\end{tabular}


Form-22_C

Introducing Pay-for-Performance (P4P) Approach to Increase Utilization of Maternal, Newborn and Child Health Services in Bangladesh

\section{Bank Reconciliation Statement}

Name of the Facility/ Cost Centre:

Month : Year:

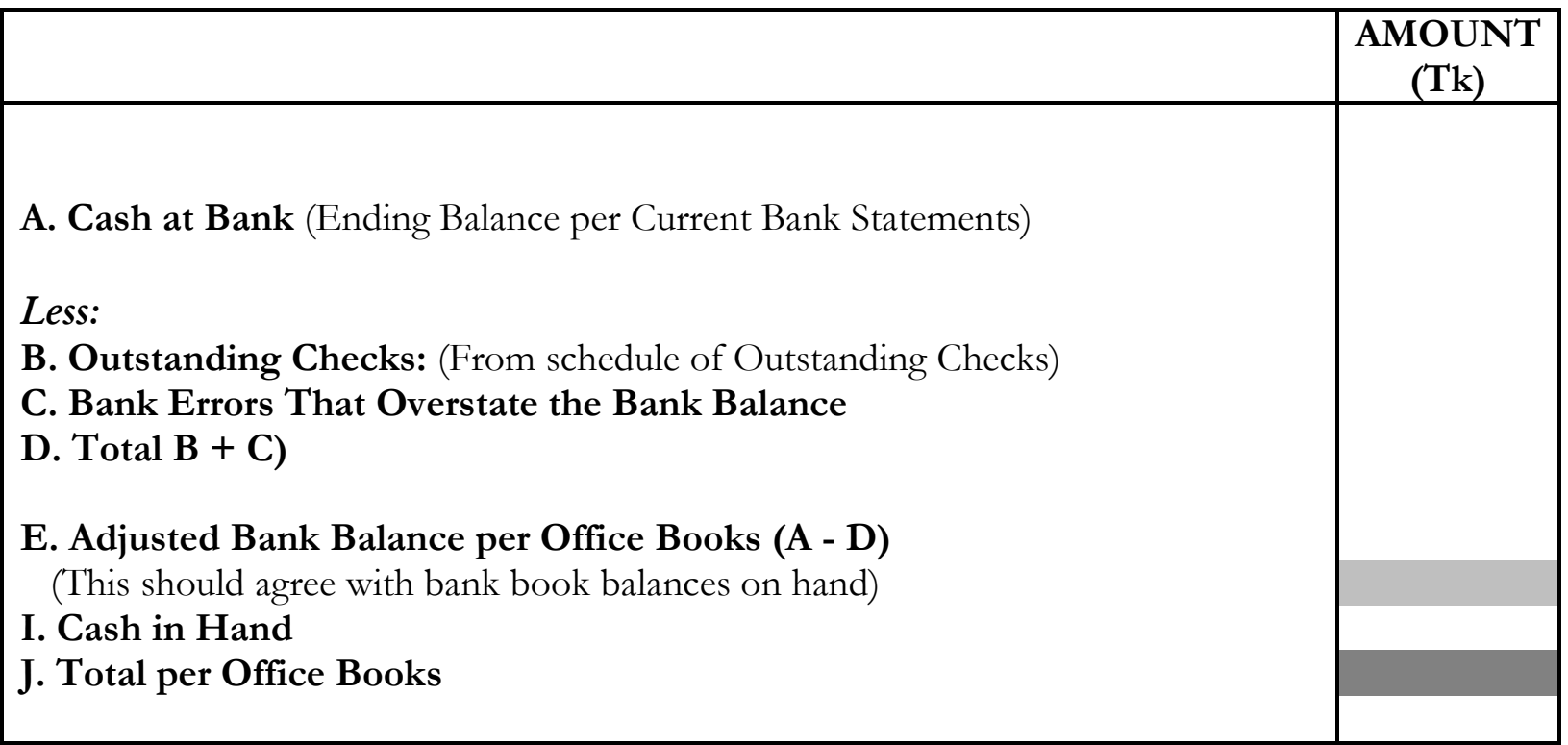


Form-22_D

Introducing Pay-for-Performance (P4P) Approach to Increase Utilization of

Maternal, Newborn and Child Health Services in Bangladesh

Name of the Facility/ Cost Centre:

\section{Statement of Receipts and Payments}

Month : Year:

1: Opening Balance Ending balance from last month's Bank Reconciliation Statement

2: Advance from PC/Dhaka:

3: Total Funds Available: (1+2)

4: Less Total Expenses:

(From Expense Account)

5: Ending Balance:

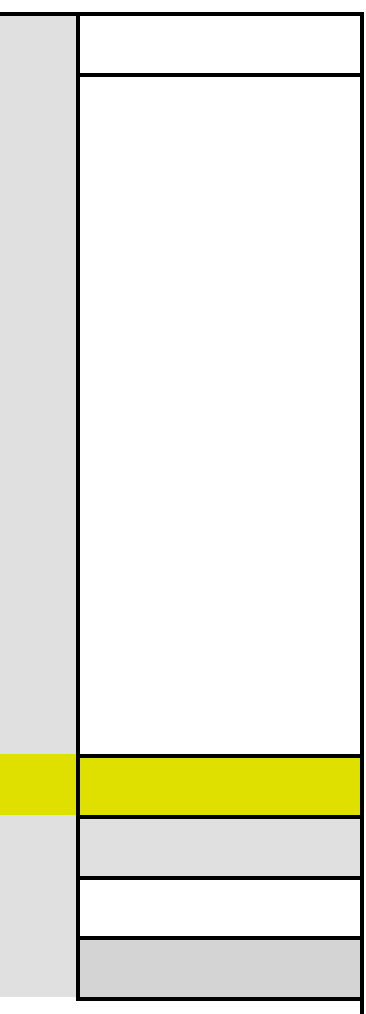

I certify that the above statement is correct:

Print Name, sign below:

Prepared by: on

Print Name, Sign below:

Approved by: on 


\section{(P Population Council}

www. popcouncil.org

South \& East Asia - Bangladesh Office House CES(B) 21, Road 118

Gulshan, Dhaka, Bangladesh

Phone : $8802-8821227,8826657$

Fax : 8802-8823127

Email : info.bangladesh@popcouncil.org 